\title{
Calibration of the Accuscan II In Vivo System for I-125 Thyroid Counting
}

\author{
O. R. Perry
}

July 2011

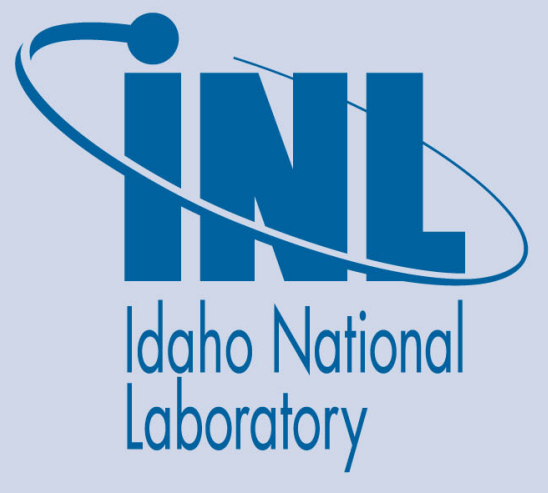

The INL is a U.S. Department of Energy National Laboratory operated by Battelle Energy Alliance 


\section{DISCLAIMER}

This information was prepared as an account of work sponsored by an agency of the U.S. Government. Neither the U.S. Government nor any agency thereof, nor any of their employees, makes any warranty, expressed or implied, or assumes any legal liability or responsibility for the accuracy, completeness, or usefulness, of any information, apparatus, product, or process disclosed, or represents that its use would not infringe privately owned rights. References herein to any specific commercial product, process, or service by trade name, trade mark, manufacturer, or otherwise, does not necessarily constitute or imply its endorsement, recommendation, or favoring by the U.S. Government or any agency thereof. The views and opinions of authors expressed herein do not necessarily state or reflect those of the U.S. Government or any agency thereof. 
INL/EXT-11-22663

Revision 0

\section{Calibration of the Accuscan II In Vivo System for I-125 Thyroid Counting}

O. R. Perry

July 2011

Idaho National Laboratory

Health Physics Dosimetry Laboratory

Idaho Falls, Idaho 83415

http://www.inl.gov

Prepared for the

U.S. Department of Energy

Office of Nuclear Energy

Under DOE Idaho Operations Office

Contract DE-AC07-05ID14517 



\section{Health Physics Dosimetry Laboratory}

\section{Calibration of the Accuscan II In Vivo System for I-125 Thyroid Counting}

INL/EXT-11-22663

Revision 0

July 2011

Approved by:

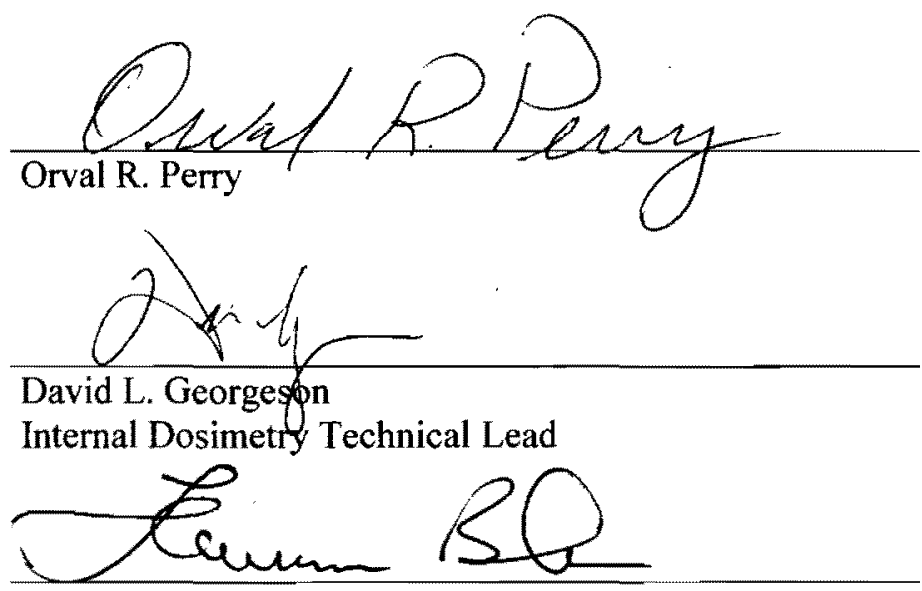

Lawrence L. Burke

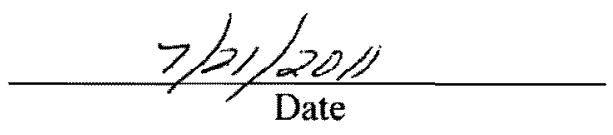

Health Physics Dosimetry Laboratory Manager

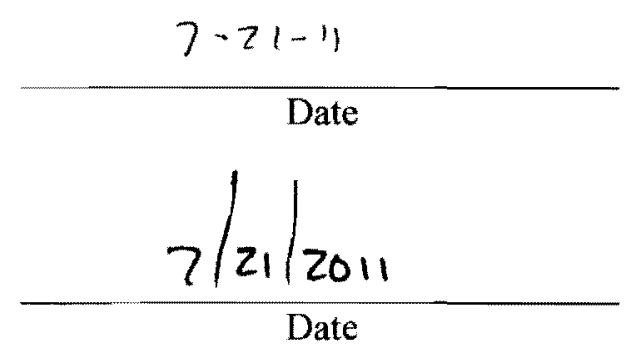





\begin{abstract}
This report describes the March 2011 calibration of the Accuscan II HpGe In Vivo system for I-125 thyroid counting. The source used for the calibration was a DOE manufactured Am-241/Eu-152 source contained in a $22 \mathrm{ml}$ vial \# BEA Am-241/Eu-152 RMC II-1 with energies from $26 \mathrm{keV}$ to $344 \mathrm{keV}$. The center of the detector housing was positioned 64" from the vault floor. This position places the approximate center line of the detector housing at the center line of the source in the phantom thyroid tube. The energy and efficiency calibration were performed using an RMC II phantom (Appendix J). Performance testing was conducted using source \# BEA Am-241/Eu-152 RMC II-1 and Validation testing was performed using an I-125 source in a $30 \mathrm{ml}$ vial (\#I-125 BEA Thyroid 002) and an ANSI N44.3 phantom (Appendix I). This report includes an overview introduction and records for the energy/FWHM and efficiency calibration including performance verification and validation counting. The Accuscan II system was successfully calibrated for counting the thyroid for I-125 and verified in accordance with ANSI/HPS N13.30-1996 criteria.
\end{abstract}




\section{CONTENTS}

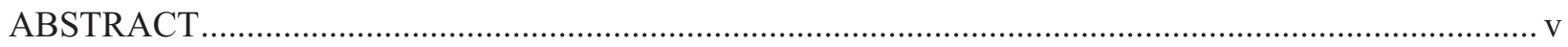

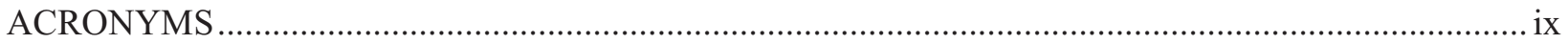

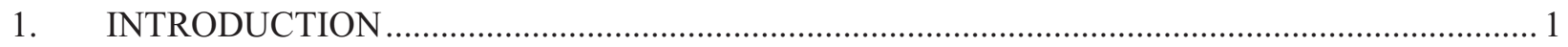

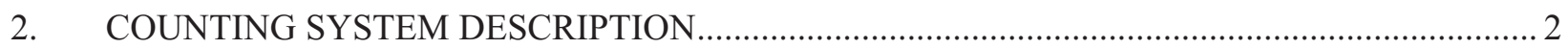

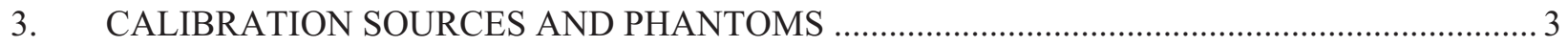

4. DETECTOR AND LYNX DIGITAL AMPLIFIER TESTING …............................................ 4

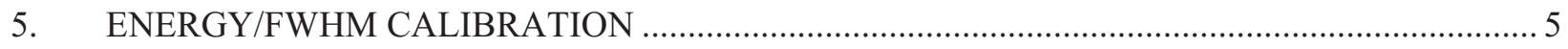

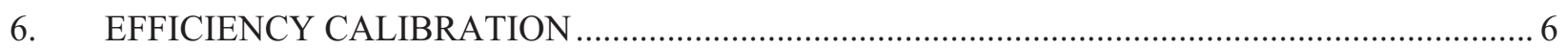

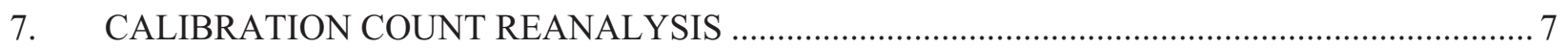

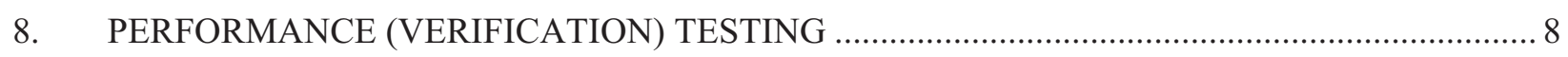

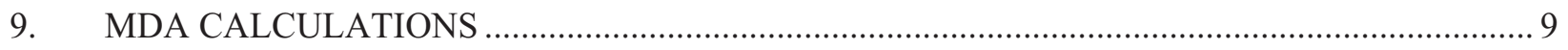

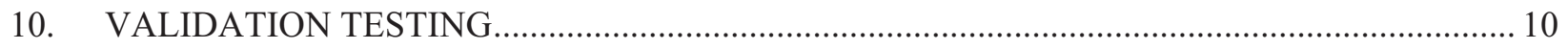

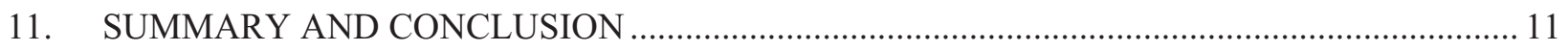

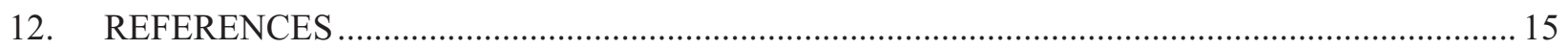

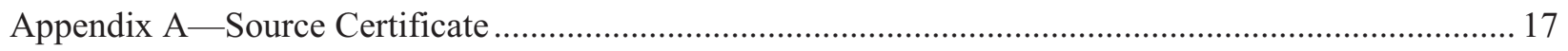

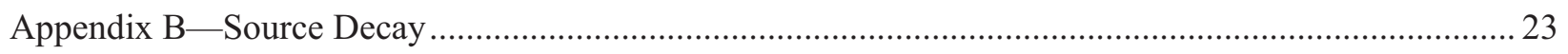

Appendix C_Energy/FWHM and Low Tail Calibration Documentation ............................................. 27

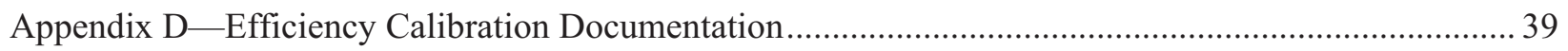

Appendix E-I-125 Calibration Count Reanalysis Summary .......................................................... 47

Appendix F-Accuscan II I-125 Calibration Verification.................................................................... 55

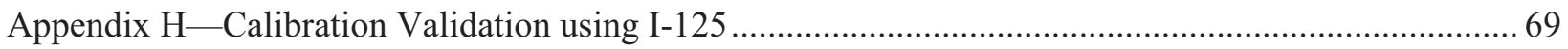

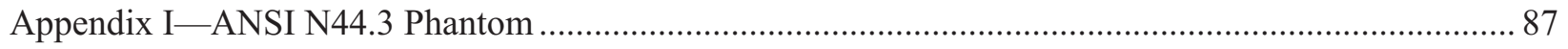

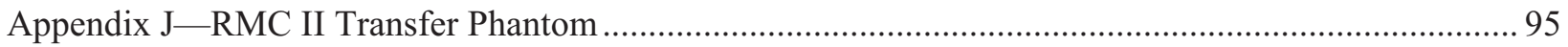




\section{FIGURES}

Figure J-1. Canberra RMS-II (Model 2257) WBC calibration phantom.

102

\section{TABLES}

Table 1. Calibration summary table.

Table 2. Testing the $27 \mathrm{keV}$ Energy Line With a Derived Yield of 43.12\%........................................... 11

Table 3. Testing the $27 \mathrm{keV}$ Energy Line with a Derived Yield of 38.25\%........................................ 12

Table 4. Testing the $27 \mathrm{keV}$ Energy Line with a Derived Yield of 40.5\% ............................................. 12

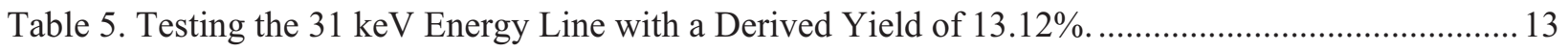

Table 6. Testing the $31 \mathrm{keV}$ Energy Line with a Derived Yield of 12.0\% ............................................. 13

Table 7. Testing the $31 \mathrm{keV}$ Energy Line with a Derived Yield of 12.5\% ............................................. 14

Table J-1. Summary of phantom comparison test count results. ......................................................... 100

Table J-2. Comparison of RMC-II and ANSI N13.30 reference phantoms efficiency ratio values and corresponding relative bias values. 


\section{ACRONYMS}

ANSI. American National Standard Institute

$A S F . \quad$ Analysis Sequence File

BE. Broad Energy

CFA Central Facility Area

CPS. Counts per second

FWHM. Full With at Half Maximum

HpGe. High Purity Germanium -also HPGe

HPL. Health Physics Laboratory

HPS. Health Physics Society

INL. Idaho National Laboratory

MDA. Minimum Detectable Activity

NIST. National Institute of Standards and Testing

PC. Personal Computer

RMC. Radiation Management Corporation

WBC Whole Body Counting 


\section{Calibration of the Accuscan II In Vivo System for I-125 Thyroid Counting}

\section{INTRODUCTION}

This document describes the March 2011 calibration of the Accuscan II in vivo system for thyroid counting of I-125. The new calibration files were stored on the system disk and made available for thyroid counting after all verification counts were completed. This is the initial calibration of the Accuscan II for personnel thyroid counting for I-125.

The energy/FWHM-Low Tail calibration was performed on March 16, 2011. A DOE manufactured $22 \mathrm{ml}$ source \# BEA Am-241/Eu-152 RMC II-1 was used together with an RMC II phantom to establish a thyroid counting geometry. This source (BEA Am-241/Eu-152 RMC II-1) is NIST traceable and is presented in Appendix A. The source has a reference date of March 14, 2011 with the current activity presented in Appendix B. The RMC II phantom was used for both the energy and efficiency calibrations. The source vial was positioned in the RMC II phantom thyroid tube and counted for 7223.15 seconds for both the energy and efficiency calibrations with the center line of the detector housing positioned 64" from the vault floor.

An efficiency calibration was performed subsequent to the energy/FWHM calibration (Appendix D). To calibrate this counting geometry, a mathematical function relating efficiency versus photopeak energy was created. The mathematical efficiency function generated on March 16, 2011 is expressed as an Empirical fit using a $4^{\text {th }}$ order polynomial with no cross over. The efficiency is determined empirically by measuring known standards and using least squares fitting techniques. The efficiency function is stored as a calibration file, which is used to analyze spectra, collected under the same counting conditions.

Replicate performance (verification) counts (using the new calibration files) were performed to confirm that the accuracy and precision of the calibration satisfied ANSI/HPS N13.30-1996 (see Reference 1) performance criteria and are presented in Appendix E.

In addition to the performance tests, a set of 5 validation counts were performed using a NIST traceable I-125 source (\# I-125 BEA Thyroid 002) manufactured by DOE. The validation counts Relative Bias and Relative Precision were tested to ANSI/HPS N13.30-1996 criteria. The results are presented in Appendix $\mathrm{H}$. 


\section{COUNTING SYSTEM DESCRIPTION}

The Accuscan II system contains two broad energy BE type HpGe detectors used for both low and high energy counting. This would include Whole Body, High Energy Lung, Thyroid, and Low Energy Lung (although at this time the system is not calibrated for energies $<26 \mathrm{keV}$ ). The Canberra Apex-InVivo software system operates from a Genie 2000 operating system on a Dell Optiplex 780 PC. The counter system is located in a 9' 4 " high $\times 8^{\prime} 71 / 2$ " wide $\times 11^{\prime} 5^{\prime}$ " deep counting vault constructed of concrete and masonry blocks. The counting system is standup with the detectors scanning from head to foot for whole body counting. For Lung and Thyroid counting the detectors are stationary at fixed counting positions. Personnel are positioned in a counting shield constructed of 4" of low background and face the detectors which are "shadow shielded" with both lead and copper. The counting vault is located on the INL site at HPL-1618. The detectors are cooled to $-185^{\circ} \mathrm{C}$ by an electrically refrigerated cryostat system. 


\section{CALIBRATION SOURCES AND PHANTOMS}

A NIST traceable DOE manufactured source \# BEA Am-241/Eu-152 RMC II-1 was used. The certification for this source is described in Appendix A. The source is uniformly distributed in a WaterWork crystal SP 400 matrix in a $22 \mathrm{ml}$ vial.

The source activity was decayed to the efficiency calibration date of March 16, 2011 by the Apex software. The Apex software also decayed the source for verification testing and presented the results in gammas per second. The phantom has been verified against the ANSI N44.3 thyroid phantom, Realistic torso phantom and Bottle Manikin Absorber model and is described in described in Appendix J.

An ANSI N44.3 phantom was used with a $30.0 \mathrm{ml}$ vial containing $101 \mathrm{nCi}$ of $\mathrm{Ba}-133$ to provide Validation testing subsequent to the calibration. The phantom is described in Appendix I.

Validation counting of the calibration was performed using DOE manufactured I-125 source \# I-125 BEA Thyroid 002. The source certification is described in Appendix A. The source is uniformly distributed in a Water Work matrix in a Liquid Scintillation Vial $(\sim 30 \mathrm{ml})$. 


\section{DETECTOR AND LYNX DIGITAL AMPLIFIER TESTING}

A Pole Zero was performed in accordance with MCP-3336 for both detectors during the I-131 calibration of March 1, 2011. No additional pole zero is needed since both calibrations use the same detector. 


\section{ENERGY/FWHM CALIBRATION}

An energy/FWHM calibration was performed March 16, 2011. The mixed The BEA Am-241/Eu-152 RMC II -1 source was positioned in the RMC II thyroid tube and counted for 7223 seconds with the detector housing midline at 64" from the vault floor. The detectors were in a fixed position for the count. The count was used to develop the energy/FWHM and Low Tail equations in accordance with MCP-3336 (Reference 2). The results and graphs of the calibrations are documented in Appendix C. 


\section{EFFICIENCY CALIBRATION}

An efficiency calibration was conducted March 16, 2011 in accordance with MCP-3336 (Reference 2) and the Canberra Apex-InVivo User's Manual (Reference 3) and documented in Appendix D. Appendix D contains the worksheets, listings, and plots for these new efficiency calibration files. The efficiency calibration count was performed using the energy calibration count described in Section 5.0. Spectral data was transferred to a disk file at the completion of the count, using "Efficiency Calibration 3/16/2011 4:31 PM" as the ID number. The efficiency calibration used library Am-241/Eu-152. NLB and ASF file CALWBCGE for I-125. 


\section{CALIBRATION COUNT REANALYSIS}

Subsequent to the energy and efficiency calibrations the calibration count (performed on 3/6/2011 4:31 PM) was analyzed using the new calibrations. The results indicated a relative bias between 0.31 and -0.01 . This result is well within the bias criteria of Reference 1. Except for the very low $26 \mathrm{keV}$ energy line of Am-241, the results also indicated an excellent calibration. The summary result and supporting documentation is attached as Appendix E. 


\section{PERFORMANCE (VERIFICATION) TESTING}

The efficiency calibration used certificate BEA Am-241/Eu-152 RMC II-1. A set of five replicate verification test of the radionuclides in certificate BEA Am-241/Eu-152 RMC II-1 were performed March 16, 2011 with the detectors positioned 64" from the vault floor and the source located in the RMC II phantom thyroid tube. These counts were analyzed using the new efficiency calibration file and the nuclide library file I-125 Thyroid.nlb. Relative bias and relative precision, as defined in ANSI/HPS N13.30-1996 (Reference 1) were calculated for these counts. Analysis results and calculated bias values for these verification counts are summarized in Appendix F. The bias ranged from $-6.9 \%$ to $1.82 \%$. The relative precision for these verification counts ranged from $12.77 \%$ to $3.56 \%$. These results are well

within the ANSI/HPS N13.30-1996 criteria ( $-25 \%$ to $+50 \%$ for the relative bias and less than or equal to $40 \%$ for the relative precision). 


\section{MDA CALCULATIONS}

MDA testing was conducted on March 23, 2011. The RMC-II phantom (with no sources installed) was counted three times with the Apex-In Vivo Analysis Sequence File (ASF) counting file (Thyroid ASF for I-125.ASF) containing the Detection Limit-Curie MDA Step. The Curie MDA equation of $\left(4.65 * S_{b}+3\right) / K T$ was installed in the step. The MDA summary and supporting documentation are attached as Appendix G. 


\section{VALIDATION TESTING}

Although the performance test indicated acceptable results based on ANSI/HPS N13.30-1996 criteria, the calibration was tested against an I-125 source manufactured by DOE as \# I-125 BEA Thyroid 002. The I-125 source was placed in the ANSI N44.3 phantom. The center of the thyroid tube of the ANSI N44.3 phantom was placed 61.25" from the counter floor, 6 9/16" from the counter rearwall and 23 7/8" from the counter right wall. The center line of the detector housing as positioned 64" from the vault floor (the counter floor is $23 / 4$ " from the vault floor). The initial test results indicated a severe under response from $46 \%$ (27 keV energy line) to $82 \%$ ( $35.5 \mathrm{keV}$ energy line). The efficiency calibration derivation was evaluated as a dual function, with varying cross over energies and polynomial values and as an empirical function with varying degrees of polynomial values. No reassessment made any significant changes to the I-125 activity resolution. The results of the low yield $35.5 \mathrm{keV}$ energy line (6.67\%) made this response vary from $-11 \%$ to $+14 \%$ and proved to be very unstable. The solution was to add a calibration constant or to adjust the yield of the energy lines to bring the reported I-125 values to the known activity level. Counting results were tested from 3/16/2011 to 3/23/2011 with a variety of yields introduced for the 27 and $31 \mathrm{keV}$ energy lines. The yield corrections varied from $38.25 \%$ to $43.12 \%$ for the $27 \mathrm{keV}$ energy line. The final yield $\%$ correction was $40.12 \%$ and was tested against all collected count data. See Tables 1,2 , and 3. The yield correction for the $31 \mathrm{keV}$ energy line varied from $11.7 \%$ to $13.12 \%$. The final correction was $12.5 \%$ and was tested against all collected count data. See Tables 4,5 , and 6 .

At the completion of establishing correction yield values for the 27 and $31 \mathrm{keV}$ energy lines, a second set of tests were conducted using the I-125 source \# I-125 BEA Thyroid 002. These tests were termed Validation Tests. The tests were conducted using the ANSI N44.3 phantom and a $30 \mathrm{ml}$ vial containing I-125. The front of the ANSI N44.3 phantom was placed 61.25" inches from the counter floor, 69/16" from the counter rearwall and $237 / 8$ " from the counter right wall. The center line of the detector housing was position 64" from the vault floor (the counter floor is approximately $23 / 4$ " from the vault floor). The results of the I-125 tests are attached as Appendix $\mathrm{H}$. The summary notes a Relative Bias of $-4.7 \%$ and a Relative Precision of $1.1 \%$ for the $27 \mathrm{keV}$ peak and a Relative Bias of $-3.2 \%$ and a Relative Precision of $7.3 \%$ for the $31 \mathrm{keV}$ peak. The average Relative Bias was $-4.0 \%$ and Relative Precision of $0.42 \%$. 


\section{SUMMARY AND CONCLUSION}

Final review of all calibration and verification count results confirmed that the thyroid calibration created on March 16, 2011 for the Accuscan II counter is satisfactory for I-125 counting where the detectors are positioned in-accordance-with Reference 4 (center of the individual's throat). A summary of current calibration files for this counter is shown in Table 7.

The I-125 Thyroid.nlb nuclide library file is the default for thyroid counting of I-125. Non-systematic and systematic errors for the in vivo system are described and quantified in Reference 5 - TEV-1275 (INL In Vivo System Counting Uncertainty).

The overall percent (\%) counting uncertainty using the Accuscan II for I-125 counting is:

Total \% Uncertainty (Accuscan II) for I - $125=\sqrt{(\% \text { counting uncertainty })^{2}+(3.63)^{2}+(18.0)^{2}}$

Table 1 . Testing the $27 \mathrm{keV}$ energy line with a derived yield of $43.12 \%$.

Testing the $27 \mathrm{keV}$ energy line with a derived yield of $43.12 \%$

\begin{tabular}{|c|c|c|c|c|c|}
\hline $\mathrm{g} / \mathrm{sec}$ & $\begin{array}{l}\text { Adjusted Yield=43.12\% } \\
\text { Activity (nCi) }\end{array}$ & $\begin{array}{l}\text { Maximum } \\
50 \% \\
\text { Limit }\end{array}$ & $\begin{array}{l}\text { Minımum } \\
-25 \% \\
\text { Limit }\end{array}$ & $\begin{array}{l}\% \text { Bias relative } \\
\text { to activity }\end{array}$ & $\begin{array}{l}\text { Test } \\
\text { Date }\end{array}$ \\
\hline 1730.08 & 108.4390513 & 163.95 & 81.975 & -0.788 & $3 / 17 / 2011$ \\
\hline 1728.91 & 108.3657173 & 163.95 & 81.975 & -0.855 & $3 / 17 / 2011$ \\
\hline 1741.08 & 109.1285163 & 163.95 & 81.975 & -0.157 & $3 / 17 / 2011$ \\
\hline 1717.89 & 107.6749987 & 163.95 & 81.975 & -1.487 & $3 / 17 / 2011$ \\
\hline 1783.97 & 111.8168029 & 163.95 & 81.975 & 2.303 & $3 / 17 / 2011$ \\
\hline 1737.93 & 108.9310786 & 163.95 & 81.975 & -0.338 & $3 / 17 / 2011$ \\
\hline 1789.18 & 112.1433586 & 163.95 & 81.975 & 2.601 & $3 / 17 / 2011$ \\
\hline 1710.19 & 107.1923733 & 163.95 & 81.975 & -1.928 & $3 / 17 / 2011$ \\
\hline 1705.97 & 106.9278694 & 163.95 & 81.975 & -2.17 & $3 / 17 / 2011$ \\
\hline 1728 & 108.3086797 & 163.95 & 81.975 & -0.907 & $3 / 17 / 2011$ \\
\hline
\end{tabular}

3/17/2011 Source Activity (nCi)

109.3 
Table 2. Testing the $27 \mathrm{keV}$ energy line with a derived yield of $38.25 \%$.

\begin{tabular}{|c|c|c|c|c|c|}
\hline gammas/sec & $\begin{array}{l}\text { Adjusted Yield=38.25\% } \\
\text { Activity (nCi) }\end{array}$ & $\begin{array}{c}\text { Maximum } \\
50 \% \\
\text { Limit }\end{array}$ & $\begin{array}{c}\text { Minimum } \\
-25 \% \\
\text { Limit }\end{array}$ & $\begin{array}{l}\text { \% Bias relative } \\
\text { to activity }\end{array}$ & $\begin{array}{l}\text { Test } \\
\text { Date }\end{array}$ \\
\hline 1439.96 & 101.7459813 & 153 & 76.5 & -0.249 & $3 / 23 / 2011$ \\
\hline 1443.81 & 102.018018 & 153 & 76.5 & 0.018 & $3 / 23 / 2011$ \\
\hline 1438.86 & 101.6682565 & 153 & 76.5 & -0.325 & $3 / 23 / 2011$ \\
\hline 1469.61 & 103.8410175 & 153 & 76.5 & 1.805 & $3 / 23 / 2011$ \\
\hline 1427.96 & 100.8980745 & 153 & 76.5 & -1.08 & $3 / 23 / 2011$ \\
\hline 1487 & 105.0697757 & 165.9 & 82.95 & -5 & $3 / 16 / 2011$ \\
\hline 1489.57 & 105.251369 & 165.9 & 82.95 & -4.836 & $3 / 16 / 2011$ \\
\hline 1498.2 & 105.8611553 & 165.9 & 82.95 & -4.285 & $3 / 16 / 2011$ \\
\hline 1445.48 & 102.1360184 & 165.9 & 82.95 & -7.653 & $3 / 16 / 2011$ \\
\hline 1515.23 & 107.0644762 & 165.9 & 82.95 & -3.197 & $3 / 16 / 2011$ \\
\hline $3 / 16 / 2011$ & Source Activity (nCi) & 110.6 & & & \\
\hline $3 / 23 / 2011$ & Source Activity (nCi) & 102 & & & \\
\hline
\end{tabular}

Table 3. Testing the $27 \mathrm{keV}$ energy line with a derived yield of $40.5 \%$.

Testing the I-125 $27 \mathrm{keV}$ Energy Line Response to an Adjusted Yield of 40.25\%

\begin{tabular}{cccc} 
gammas/sec & $\mathrm{nCi}$ & Test Date & \\
\hline 1730.08 & 116.1712271 & $3 / 17 / 2011$ & 6.287 \\
1728.91 & 116.0926641 & $3 / 17 / 2011$ & 6.215 \\
1741.08 & 116.909854 & $3 / 17 / 2011$ & 6.962 \\
1717.89 & 115.3526943 & $3 / 17 / 2011$ & 5.538 \\
1783.97 & 119.7898271 & $3 / 17 / 2011$ & 9.597 \\
1737.93 & 116.6983381 & $3 / 17 / 2011$ & 6.769 \\
1789.18 & 120.1396676 & $3 / 17 / 2011$ & 9.917 \\
1710.19 & 114.8356555 & $3 / 17 / 2011$ & 5.065 \\
1705.97 & 114.5522914 & $3 / 17 / 2011$ & 4.805 \\
1728 & 116.0315595 & $3 / 17 / 2011$ & 6.159 \\
1439.96 & 96.69028034 & $3 / 23 / 2011$ & -5.206 \\
1443.81 & 96.94879973 & $3 / 23 / 2011$ & -4.952 \\
1438.86 & 96.61641766 & $3 / 23 / 2011$ & -5.278 \\
1469.61 & 98.68121538 & $3 / 23 / 2011$ & -3.254 \\
1427.96 & 95.88450562 & $3 / 23 / 2011$ & -5.996 \\
1487 & 99.84891724 & $3 / 16 / 2011$ & -9.721 \\
1489.57 & 100.0214873 & $3 / 16 / 2011$ & -9.565 \\
1498.2 & 100.6009736 & $3 / 16 / 2011$ & -9.041 \\
1445.48 & 97.06093671 & $3 / 16 / 2011$ & -12.241 \\
1515.23 & 101.7445023 & $3 / 16 / 2011$ & -8.007 \\
$3 / 17 / 2011$ & & \\
$3 / 16 / 2011$ & Source Activity $(\mathrm{nCi})$ & 109.3 & \\
$3 / 23 / 2011$ & Source Activity $(\mathrm{nCi})$ & 110.6 & \\
& & 102 &
\end{tabular}


Table 4 . Testing the $31 \mathrm{keV}$ energy line with a derived yield of $13.12 \%$.

Testing the $31 \mathrm{keV}$ energy line with a derived yield of $13.12 \%$

\begin{tabular}{|c|c|c|c|c|c|}
\hline $\mathrm{g} / \mathrm{sec}$ & $\begin{array}{l}\text { Adjusted Yield=13.12\% } \\
\text { Activity (nCi) }\end{array}$ & $\begin{array}{c}\text { Maximum } \\
50 \% \\
\text { Limit }\end{array}$ & $\begin{array}{c}\text { Minimum } \\
-25 \% \\
\text { Limit }\end{array}$ & $\begin{array}{l}\% \text { Bias relative } \\
\text { to activity }\end{array}$ & $\begin{array}{l}\text { Test } \\
\text { Date }\end{array}$ \\
\hline 510.36 & 105.1334871 & 163.95 & 81.975 & -3.812 & $3 / 17 / 2011$ \\
\hline 542.8 & 111.8160844 & 163.95 & 81.975 & 2.302 & $3 / 17 / 2011$ \\
\hline 538.41 & 110.9117502 & 163.95 & 81.975 & 1.475 & $3 / 17 / 2011$ \\
\hline 544.07 & 112.0777027 & 163.95 & 81.975 & 2.541 & $3 / 17 / 2011$ \\
\hline 537.45 & 110.7139914 & 163.95 & 81.975 & 1.294 & $3 / 17 / 2011$ \\
\hline 537.34 & 110.6913316 & 163.95 & 81.975 & 1.273 & $3 / 17 / 2011$ \\
\hline 556.63 & 114.6650461 & 163.95 & 81.975 & 4.909 & $3 / 17 / 2011$ \\
\hline 519.98 & 107.1151945 & 163.95 & 81.975 & -1.999 & $3 / 17 / 2011$ \\
\hline 503.42 & 103.7038563 & 163.95 & 81.975 & -5.12 & $3 / 17 / 2011$ \\
\hline 497.22 & 102.4266645 & 163.95 & 81.975 & -6.289 & $3 / 17 / 2011$ \\
\hline
\end{tabular}

3/17/2011 Source Activity (nCi)

109.3

Table 5. Testing the $31 \mathrm{keV}$ energy line with a derived yield of $12.0 \%$.

Testing the $31 \mathrm{keV}$ energy line with a derived yield of $12.0 \%$

\begin{tabular}{|c|c|c|c|c|c|}
\hline $\mathrm{g} / \mathrm{sec}$ & $\begin{array}{l}\text { Adjusted Yield }=12.0 \% \\
\text { Activity (nCi) }\end{array}$ & $\begin{array}{c}\text { Maximum } \\
50 \% \\
\text { Limit }\end{array}$ & $\begin{array}{l}\text { Minimum } \\
-25 \% \\
\text { Limit } \\
\end{array}$ & $\begin{array}{l}\% \text { Bias relative } \\
\text { to activity }\end{array}$ & $\begin{array}{l}\text { Test } \\
\text { Date }\end{array}$ \\
\hline 367.39 & 82.7454955 & 153 & 76.5 & -18.877 & $3 / 23 / 2011$ \\
\hline 480.42 & 108.2027027 & 153 & 76.5 & 6.081 & $3 / 23 / 2011$ \\
\hline 459.42 & 103.472973 & 153 & 76.5 & 1.444 & $3 / 23 / 2011$ \\
\hline 439.25 & 98.93018018 & 153 & 76.5 & -3.01 & $3 / 23 / 2011$ \\
\hline 466.55 & 105.0788288 & 153 & 76.5 & 3.018 & $3 / 23 / 2011$ \\
\hline 517.79 & 116.6193694 & 153 & 76.5 & 14.333 & $3 / 23 / 2011$ \\
\hline 418.26 & 94.2027027 & 153 & 76.5 & -7.644 & $3 / 23 / 2011$ \\
\hline 451.73 & 101.740991 & 153 & 76.5 & -0.254 & $3 / 23 / 2011$ \\
\hline 378.9 & 85.33783784 & 153 & 76.5 & -16.335 & $3 / 23 / 2011$ \\
\hline 508.73 & 114.5788288 & 153 & 76.5 & 12.332 & $3 / 23 / 2011$ \\
\hline $3 / 23 / 2011$ & ource Activity (nCi) & 102 & & & \\
\hline
\end{tabular}


Table 6. Testing the $31 \mathrm{keV}$ energy line with a derived yield of $12.5 \%$.

Testing the I-125 $31 \mathrm{keV}$ Energy Line Response to an Adjusted Yield of 12.5\%

\begin{tabular}{cccc} 
gammas/sec & $\mathrm{nCi}$ & Test Date & \\
\hline 510.36 & 110.3481081 & $3 / 17 / 2011$ & Bias $\%$ \\
542.8 & 117.3621622 & $3 / 17 / 2011$ & 7.959 \\
538.41 & 116.412973 & $3 / 17 / 2011$ & 6.508 \\
544.07 & 117.6367568 & $3 / 17 / 2011$ & 7.627 \\
537.45 & 116.2054054 & $3 / 17 / 2011$ & 6.318 \\
537.34 & 116.1816216 & $3 / 17 / 2011$ & 6.296 \\
556.63 & 120.3524324 & $3 / 17 / 2011$ & 10.112 \\
519.98 & 112.4281081 & $3 / 17 / 2011$ & 2.862 \\
503.42 & 108.8475676 & $3 / 17 / 2011$ & -0.414 \\
497.22 & 107.507027 & $3 / 17 / 2011$ & -1.64 \\
367.39 & 79.43567568 & $3 / 23 / 2011$ & -22.122 \\
480.42 & 103.8745946 & $3 / 23 / 2011$ & 1.838 \\
459.42 & 99.33405405 & $3 / 23 / 2011$ & -2.614 \\
439.25 & 94.97297297 & $3 / 23 / 2011$ & -6.889 \\
466.55 & 100.8756757 & $3 / 23 / 2011$ & -1.102 \\
517.79 & 111.9545946 & $3 / 23 / 2011$ & 9.759 \\
418.26 & 90.43459459 & $3 / 23 / 2011$ & -11.339 \\
451.73 & 97.67135135 & $3 / 23 / 2011$ & -4.244 \\
378.9 & 81.92432432 & $3 / 23 / 2011$ & -19.682 \\
508.73 & 109.9956757 & $3 / 23 / 2011$ & 7.839 \\
& & & \\
$3 / 17 / 2011$ & Source Activity (nCi) & 109.3 & \\
$3 / 23 / 2011$ & Source Activity (nCi) & 102 &
\end{tabular}

Table 7. Calibration summary table.

\begin{tabular}{lcc}
\hline $\begin{array}{l}\text { Facility: } \\
\text { Counting System: } \\
\text { Counter Arrangement: }\end{array}$ & $\begin{array}{l}\text { Idaho National Laboratory, Building HPDL CFA-1618 } \\
\text { Accuscan II Thyroid Stationary Position } \\
\text { Accuscan II for I-125 }\end{array}$ \\
\hline \multicolumn{1}{c}{ Detector } & Energy Calibration Date & Time \\
\hline Accuscan II BE Detectors & $03 / 16 / 2011$ & $3: 02$ PM \\
Efficiency Calibration: & Energy Calibration Date & Time \\
\hline \multicolumn{1}{c}{ Detector } & $03 / 16 / 2011$ & $4: 31$ PM \\
\hline Accuscan II BE Detectors & & \\
\hline
\end{tabular}




\section{REFERENCES}

1. American National Standard, American National Standards Institute/Health Physics Society ANSI/HPS N13.30-1996, "Performance Criteria for Radiobioassay," 1996.

2. Idaho National Laboratory, Management Control Procedure MCP-3336, “Accuscan II Calibration, Proficiency Testing, and Reporting," Health Physics Dosimetry Laboratory, Revision 0, March 2011.

3. Canberra Industries Inc., 70655586, V.1, “Apex-InVivo Productivity Software User’s Manual.

4. Idaho National Laboratory, Basis Document TEV-1273, "Basis for Positioning of the In Vivo Accuscan II Detectors ”, 2011.

5. Idaho National Laboratory, Basis Document TEV-1275, "INL In Vivo System Counting Uncertainty", 2011. 
Appendix A

\section{Source Certificate}




\section{Appendix A \\ Source Certificate}

BEA Am-241/Eu-152 RMC II-1

\section{Certified גieference Atateríal}

Certificate of Traceability

\section{U.S. Department of Energy \\ Radiological and Environmental Sciences Laboratory}

Standard Identification: BEA Am-241/Ea-152 RMCII-1

Radionuclide

Activity
Eu- 152
$(1.799 \pm 0.015) \mathrm{E} 2 \mathrm{nCi}$
An-241
(2.10 10.04$) \mathrm{F} 2 \mathrm{nCi}$

This Certified Referenee Material (CRM) eontains a known quantity of radionuelides in a stable and honogeneous matrix. This material is intended for the calibration of instruments and for the verification of the accuracy and precision of analytical radiochem istry measurements. The activities listed for ench radionuclide are traceable to NIST through an un broken chain of comparisons, all having stated encertaint ies $(\mathrm{k}=1)$ calculated according to ISO and NIST Guidelines.

This material was prepared by the Radiological and Environmental Seiences Laboratory (RESL). RESL maintains direct traceability to NIST through successful participation in the NIST/RESL Radiological Traceability Program and is aceredited to ISO 17043 as a Performance Testing Provider and ISO 17025 as a Chemical Testing Laboratory by The Anerican Association for Laboratory Accrediation.

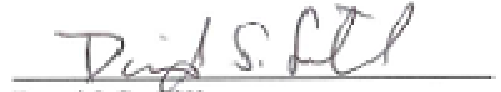

David S. Sill

Senior Technical Manager - Chemistry

March 14, 2011

Reference Date

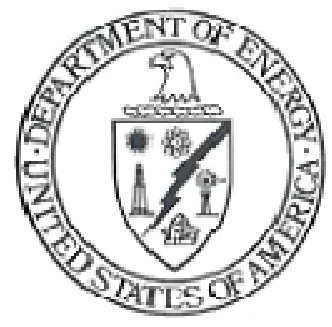


Supplemental Information

\section{BEA Am-241/Eu-152 RMCII-1}

Final weight of vial with s.icker and tape: 38.790 grams

Weight of solution in vial: $19.599+/-0.002$ grams

Solution in vial: Solidified with 0.369 grams of WaterWorks Crystal SP400.

Half life Am-241: $4.327 E+02$ years

Half life Eu-152: 1.348E+01 years 
DOE Source Certificate I-125 BEA Thyroid 002

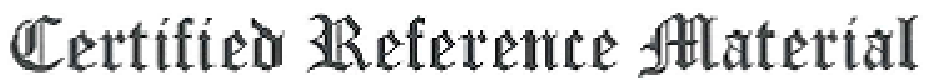

Certificate of Traceability

\section{U.S. Department of Energy \\ Radiological and Environmental Sciences Laboratory}

Standard Identification: I-125 BEA Thyroid 002

Radionuclide

Activity

$\mathrm{I}=125$

$(1.33 \pm 0.02) \mathrm{E} 2 \mathrm{nCi}$

This Certified Reference Material (CRM) contains a known quantity of radionuclides in a stable and homogeneous matrix. This material is intended for the ealibration of instruments and for the verification of the accuracy and precision of analytical radiochemisiry measurements. The activities listed for each radionuclide ate traceable to NIST" through an unoroken chain of conparisons, all having stated tincertaintics (k=1) calculated according to ISO and NIST Guidelines.

This matcrial was prepared by the Radiologieal and Environmental Scienecs Laboratory (RESSL.). RESL. maintains direct traceability to NIST through snecessful participation in the NIST/RESL Radiological Traceability Progiam and is accredited to ISO 17043 as a Per formance Testing Provider and ISO 17025 as a Chemical Testing Laboratory by The American Association for Laboratory Aecreditation.

David S. Sill

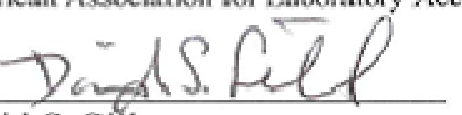

Senior Technical Manager - Chenistry

February 28,2011

Reference Date

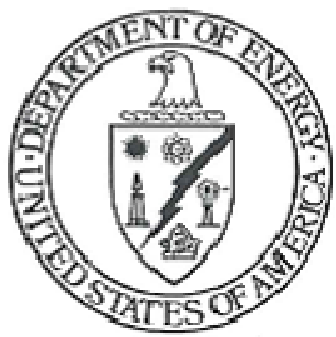




\section{Supplemental Information}

\section{I-125 BEA Thyroid 002}

Final weight of vial with sticker and tape: 38.885 grams

Weight of solution in vial: $29.955+/-0.002$ grams

Solution Used: I-125-80943-370

Solution in vial: Solidified with 0.432 grams of WaterWorks Crystal SP400.

Half life: 59.40 days

Certificate and dilution information attached.

Prepared by Guy Backstrom, 2/28/2011 


\section{Appendix B}

\section{Source Decay}




\section{Appendix B Source Decay}

\section{BEA Am-241/Eu-152 RMC II-1 Source Decay}

\begin{tabular}{|c|c|c|c|c|c|c|c|c|}
\hline Am241/Eu152 R & 1C II-1 & & & & & & & \\
\hline Reference Date & $3 / 14 / 2011$ & & & & & & & \\
\hline Current Date & $3 / 16 / 2011$ & & & & & & & \\
\hline Elapsed Time & 2 & & & & & & & \\
\hline & & Reference & & & & & & Current \\
\hline Identity & Energy (keV) & Activity (nCi) & Yield \% & $\begin{array}{l}\mathrm{T} 1 / 2 \\
\text { Days }\end{array}$ & $\mathrm{G} / \mathrm{nCi}$ & & $\begin{array}{l}\text { Reference } \\
\text { GPS }\end{array}$ & $\begin{array}{l}\text { Activity } \\
\text { (nCi) }\end{array}$ \\
\hline & 13.93 & 210 & 24.4 & 157835.5 & & 37 & $1.90 \mathrm{E}+03$ & $2.10 \mathrm{E}+02$ \\
\hline & 17.51 & 210 & 30.5 & 157835.5 & & 37 & $2.37 \mathrm{E}+03$ & $2.10 \mathrm{E}+02$ \\
\hline & 20.98 & 210 & 7.31 & 157835.5 & & 37 & $5.68 \mathrm{E}+02$ & $2.10 \mathrm{E}+02$ \\
\hline Am-241 & 26.34 & 210 & 2.4 & 157835.5 & & 37 & $1.86 \mathrm{E}+02$ & $2.10 \mathrm{E}+02$ \\
\hline & 59.54 & 210 & 35.7 & 157835.5 & & 37 & $2.77 E+03$ & $2.10 E+02$ \\
\hline Eu-152 & 121.783 & 179.9 & 28.4 & 4946.2 & & 37 & $1.89 \mathrm{E}+03$ & $1.80 \mathrm{E}+02$ \\
\hline & 244.699 & 179.9 & 7.51 & 4946.2 & & 37 & $5.00 E+02$ & $1.80 \mathrm{E}+02$ \\
\hline & 344.281 & 179.9 & 26.6 & 4946.2 & & 37 & $1.77 E+03$ & $1.80 \mathrm{E}+02$ \\
\hline
\end{tabular}




\section{DOE Manufactured Source \# I-125 BEA Thyroid 002 Decay}

I-125 BEA Thyroid 002

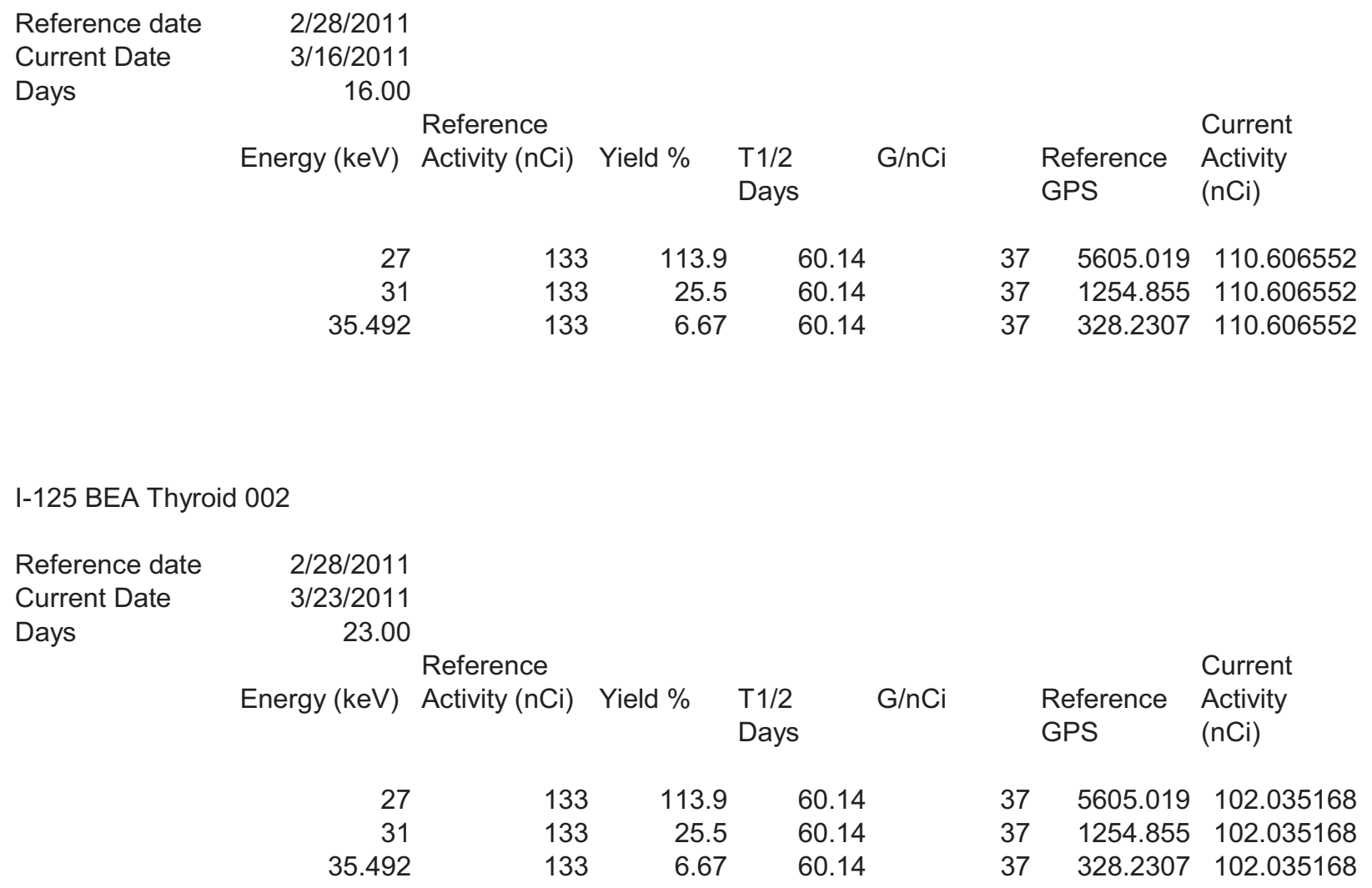


Appendix C

\section{Energy/FWHM and Low Tail Calibration Documentation}




\section{Appendix C \\ Energy/FWHM and Low Tail \\ Calibration Documentation}

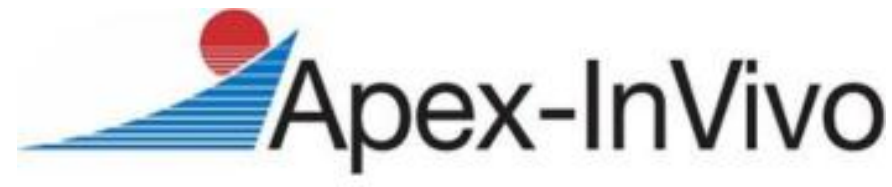

\section{Energy Calibration}

\section{Calibration Information}

$\begin{array}{ll}\text { Calibration Title } & \text { Energy Calibration (I-125) 3/16/2011 3:00 PM } \\ \text { Calibration Date } & 3 / 16 / 2011 \text { 3:01:08 PM } \\ \text { Performed By } & \text { David Georgeson } \\ \text { Count Used } & \text { Calibration Count 3/16/2011 12:56 PM } \\ \text { Is Default } & \text { Yes }\end{array}$

\section{Counter Information}

$\begin{array}{ll}\text { Counter Name } & \text { Accuscan II } \\ \text { Counter Location } & \text { Battelle }\end{array}$

\section{Detector Group Information}

Detector Group Name

Calibration Source

Analysis Sequence File

Nuclide Library

Peak Search Library
Left

Am241/Eu152 RMC II-1

Am-241/Eu-152.NLB

Am-241/Eu-152.NLB

\section{Energy Calibration Coefficients}

$$
\begin{aligned}
\text { Energy } & =-0.375273+0.462746 * \text { ch }-6.01373 \mathrm{e}-07 * \mathrm{ch} \wedge 2 \\
\text { FWHM } & =1.14092+0.0173155 * \mathrm{E}^{\wedge} 1 / 2 \\
\text { LOW TAIL } & =4.01565-0.012525 * \mathrm{E}
\end{aligned}
$$

\section{Energy Calibration Results Table}

\begin{tabular}{rrr} 
Centroid Channel & Centroid Error & Energy $(\mathbf{k e V})$ \\
\cline { 2 - 3 } 57.41 & 0.25 & 26.34 \\
\hline 129.51 & 0.01 & 59.54 \\
\hline 264.06 & 0.01 & 121.78 \\
\hline 530.03 & 0.02 & 244.70 \\
\hline 745.52 & 0.01 & 344.28
\end{tabular}

\section{Approved by:}

Date: 


\section{Energy}

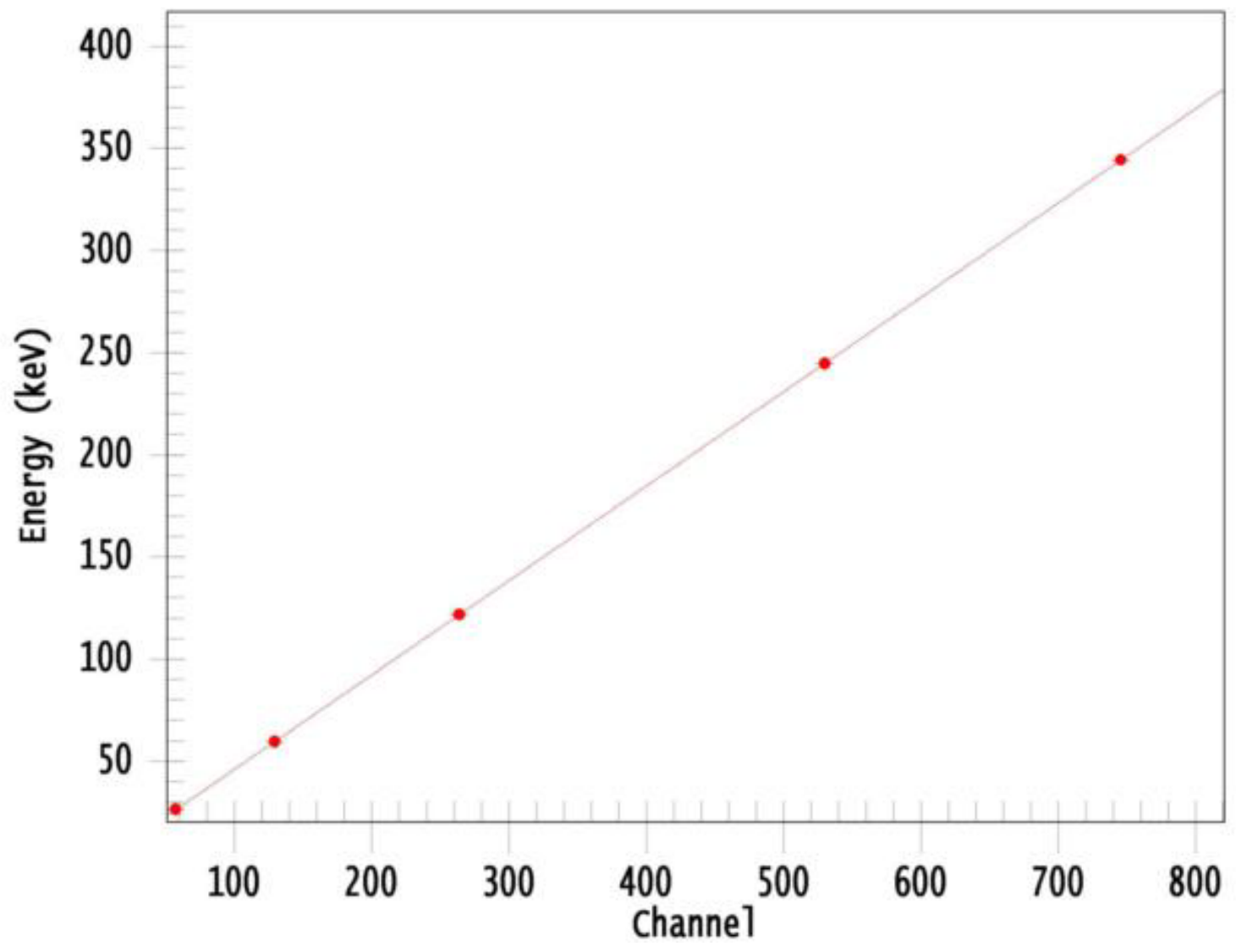

Energy Calibration Results Table

\begin{tabular}{rrr} 
Energy $(\mathbf{k e V})$ & FWHM Channels & FWHM Error \\
\cline { 2 - 3 } 26.34 & 2.24 & 0.54 \\
\hline 59.54 & 2.75 & 0.02 \\
\hline 121.78 & 2.89 & 0.02 \\
\hline 244.70 & 2.96 & 0.05 \\
\hline 344.28 & 3.17 & 0.03
\end{tabular}



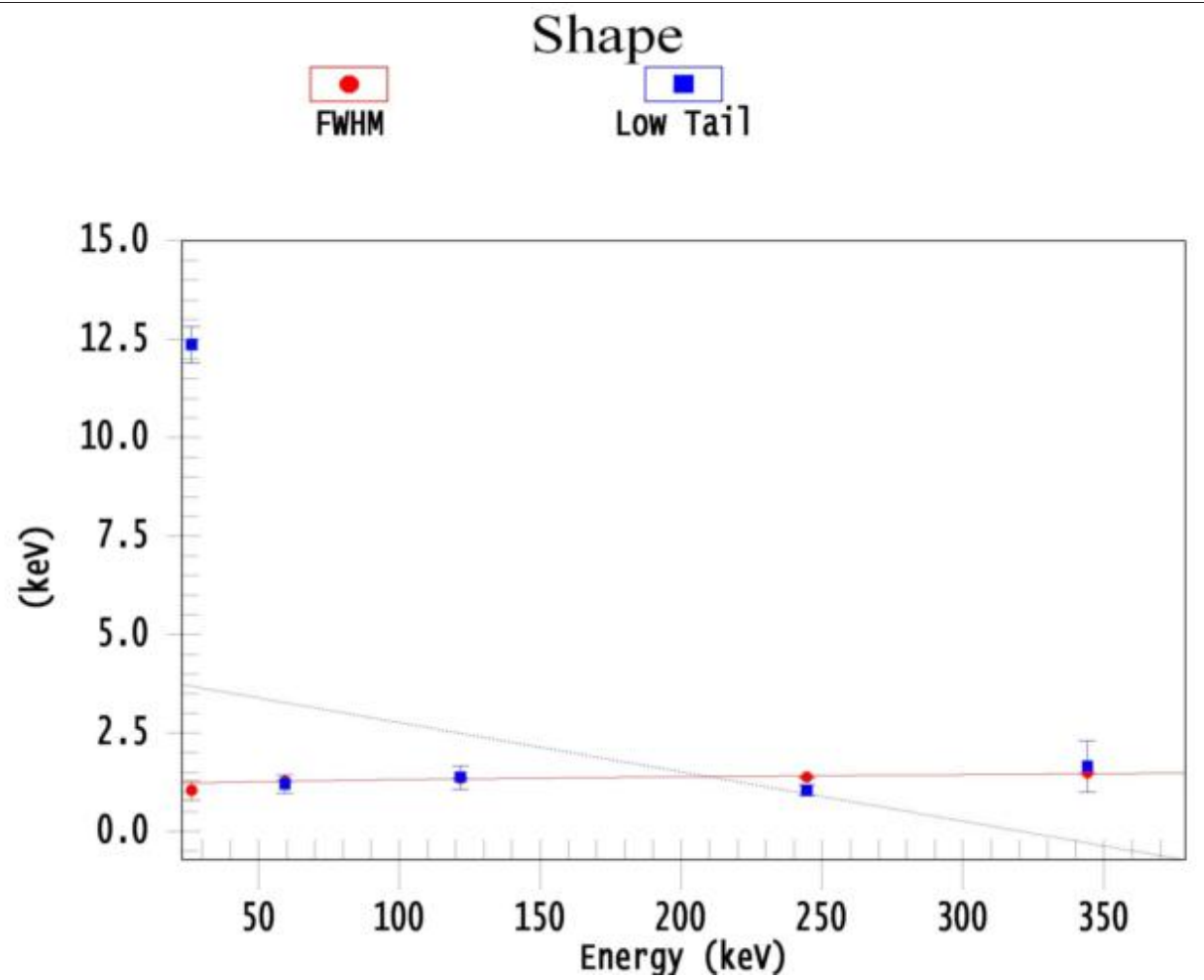

User: David Georgeson 


\section{Energy Calibration}

\section{Calibration Information}

\section{Calibration Title \\ Calibration Date \\ Performed By \\ Count Used \\ Is Default}

Energy Calibration (I-125)3/16/2011 3:02 PM

3/16/2011 3:02:15 PM

David Georgeson

Calibration Count 3/16/2011 12:56 PM

Yes

\section{Counter Information}

Counter Name

Counter Location

Accuscan II

Battelle

Detector Group Information

Detector Group Name
Calibration Source
Analysis Sequence File
Nuclide Library
Peak Search Library

Right

Am241/Eu152 RMC II-1

Am-241/Eu-152.NLB

Am-241/Eu-152.NLB

\section{Energy Calibration Coefficients}

$$
\begin{aligned}
\text { Energy } & =-0.346161+0.46233 * \text { ch }-2.30088 \mathrm{e}-07 * \mathrm{ch}^{\wedge} 2 \\
\text { FWHM } & =0.631027+0.0248655 * \mathrm{E}^{\wedge} 1 / 2 \\
\text { LOW TAIL } & =0.519347+0.00136678 * \mathrm{E}
\end{aligned}
$$

\section{Energy Calibration Results Table}

\begin{tabular}{rrr} 
Centroid Channel & Centroid Error & Energy $(\mathbf{k e V})$ \\
\cline { 2 - 3 } 57.52 & 0.12 & 26.34 \\
\hline 129.55 & 0.01 & 59.54 \\
\hline 264.19 & 0.01 & 121.78 \\
\hline 530.19 & 0.02 & 244.70 \\
\hline 745.69 & 0.01 & 344.28 \\
\hline
\end{tabular}

Approved by:

Date: 


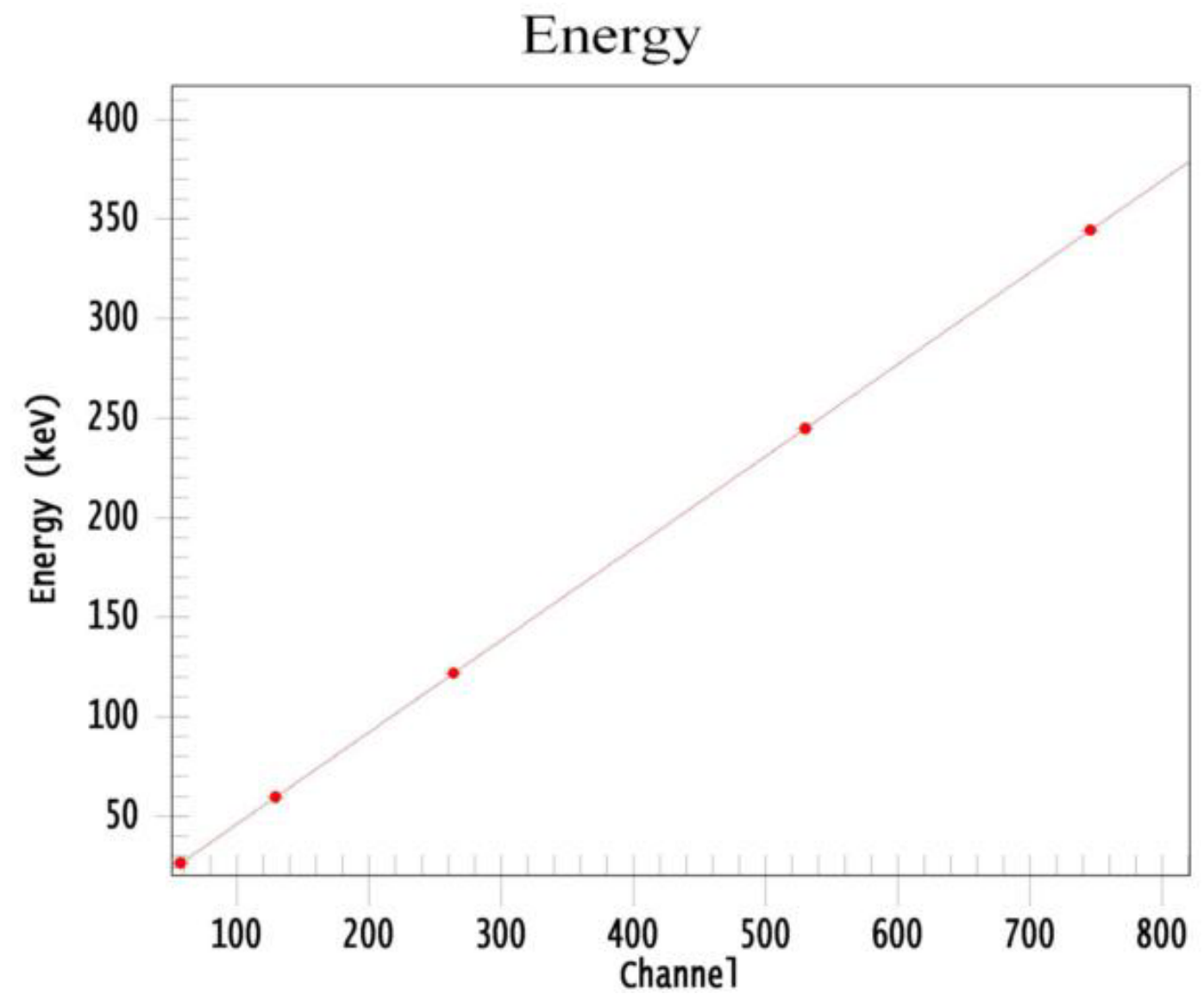

\section{Energy Calibration Results Table}

\begin{tabular}{|c|c|c|c|c|}
\hline Energy (keV) & FWHM Channels & FWHM Error & TAIL Channels & TAIL Error \\
\hline 26.34 & 1.38 & 0.71 & 0.53 & 0.60 \\
\hline 59.54 & 1.81 & 0.03 & 1.23 & 0.12 \\
\hline 121.78 & 1.94 & 0.02 & 1.76 & 0.18 \\
\hline 244.70 & 2.25 & 0.04 & 2.07 & 1.02 \\
\hline 344.28 & 2.36 & 0.02 & 2.05 & 0.21 \\
\hline
\end{tabular}




\section{Shape}
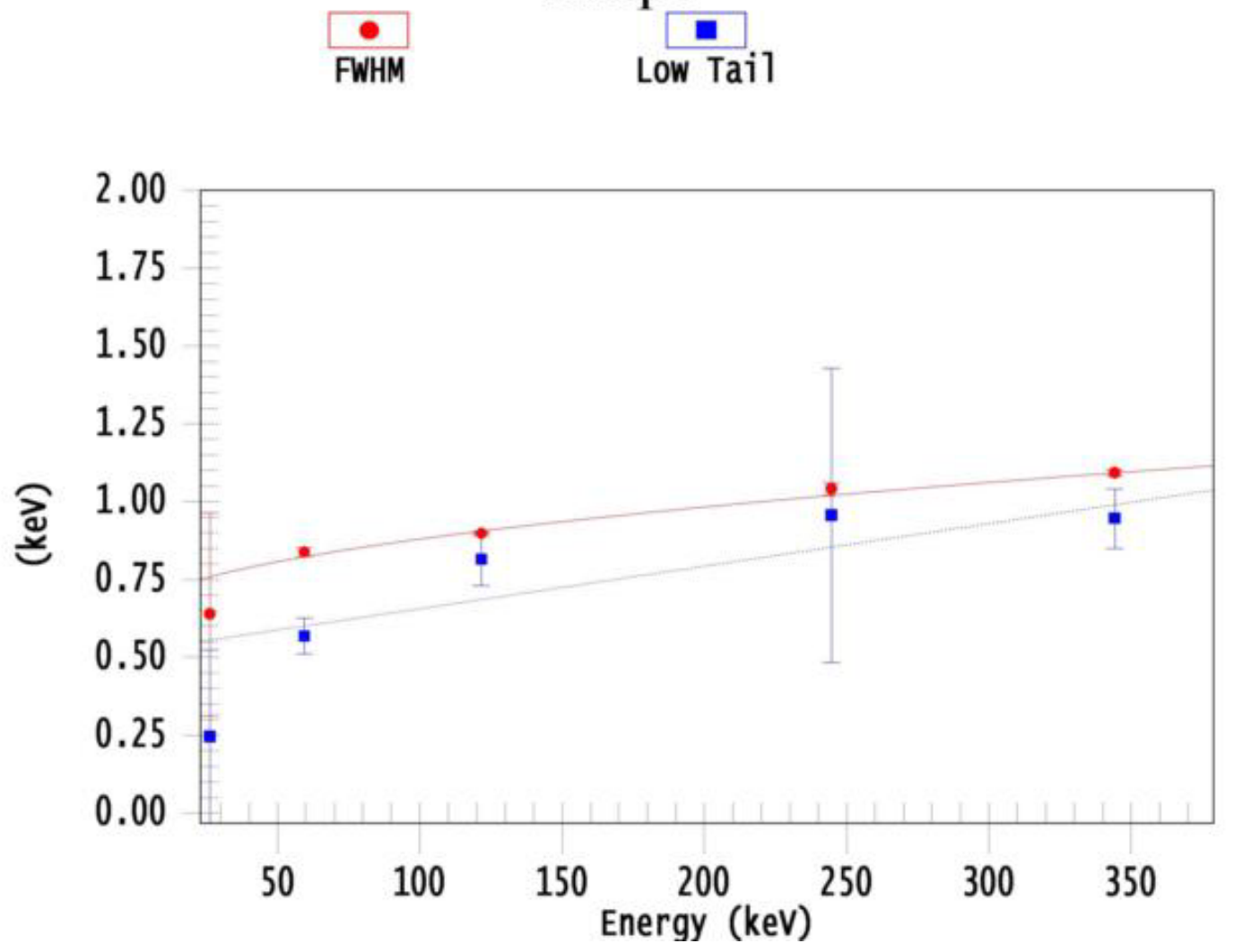


\section{Energy Calibration}

\section{Calibration Information}

Calibration Title

Calibration Date

Performed By

Count Used

Is Default
Energy Calibration (I-125) 3/16/2011 3:02 PM

3/16/2011 3:03:00 PM

David Georgeson

Calibration Count 3/16/2011 12:56 PM

Yes

\section{Counter Information}

Counter Name

Counter Location

Accuscan II

Battelle

Detector Group Information

\section{Detector Group Name}

Calibration Source

Analysis Sequence File

Nuclide Library

Peak Search Library

\section{Summed}

Am241/Eu152 RMC II-1

Am-241/Eu-152.NLB

Am-241/Eu-152.NLB

\section{Energy Calibration Coefficients}

$$
\begin{aligned}
\text { Energy } & =-0.365335+0.46262 * \mathrm{ch}-4.49624 \mathrm{e}-07 * \mathrm{ch}^{\wedge} 2 \\
\text { FWHM } & =0.848541+0.025188 * \mathrm{E}^{\wedge} 1 / 2 \\
\text { LOW TAIL } & =0.623077+0.00135443 * \mathrm{E}
\end{aligned}
$$

\section{Energy Calibration Results Table}

\begin{tabular}{rrr} 
Centroid Channel & Centroid Error & Energy $(\mathbf{k e V})$ \\
\cline { 2 - 3 } 57.52 & 0.17 & 26.34 \\
\hline 129.52 & 0.01 & 59.54 \\
264.08 & 0.02 & 121.78 \\
\hline 530.03 & 0.02 & 244.70 \\
\hline 745.53 & 0.01 & 344.28 \\
\hline
\end{tabular}

Approved by:

Date: 


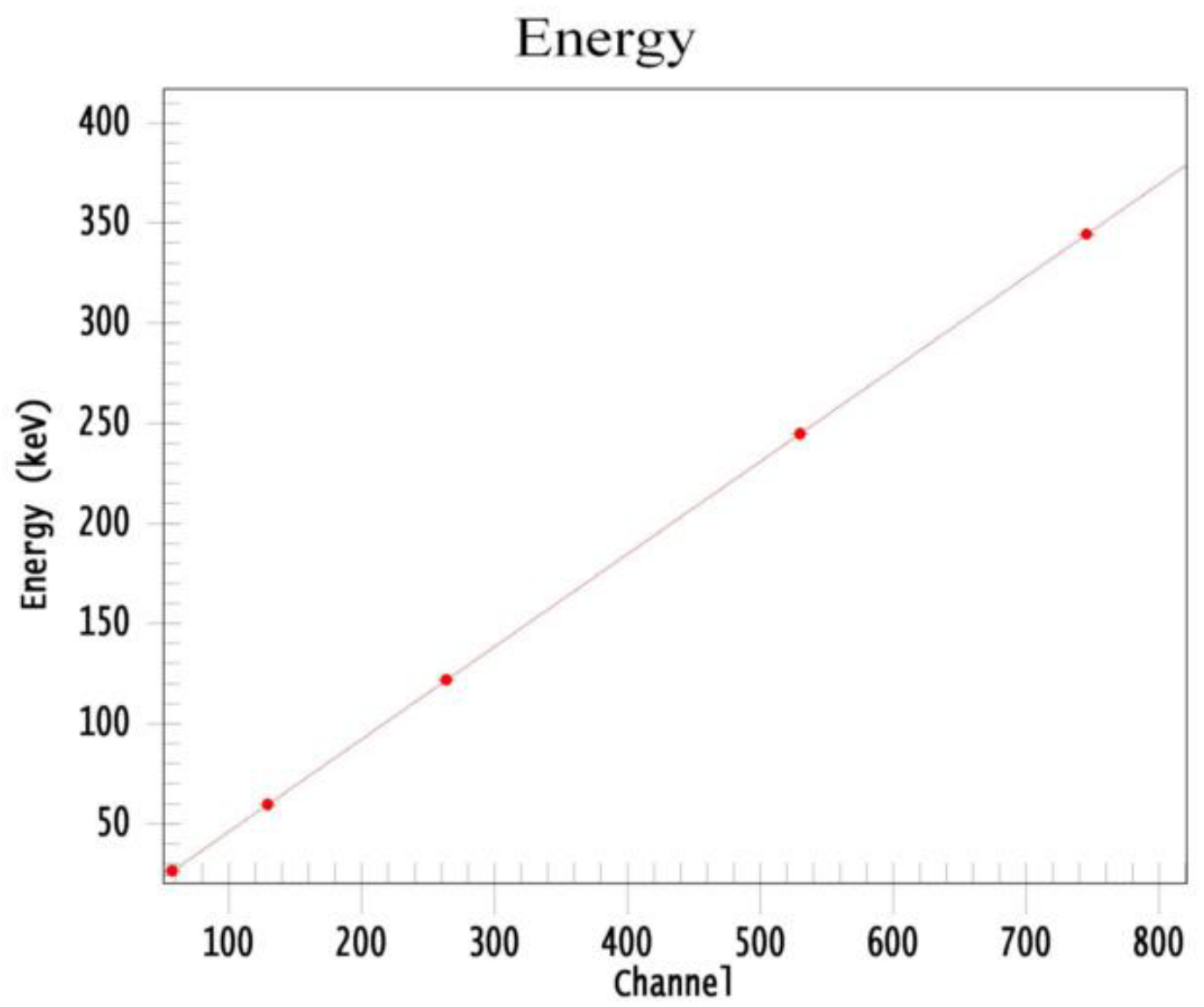

\section{Energy Calibration Results Table}

\begin{tabular}{|c|c|c|c|c|}
\hline Energy (keV) & FWHM Channels & FWHM Error & TAIL Channels & TAIL Error \\
\hline 26.34 & 1.76 & 0.52 & 0.69 & 0.57 \\
\hline 59.54 & 2.26 & 0.03 & 1.59 & 0.16 \\
\hline 121.78 & 2.43 & 0.04 & 1.70 & 0.18 \\
\hline 244.70 & 2.68 & 0.04 & 2.00 & 0.27 \\
\hline 344.28 & 2.85 & 0.02 & 2.64 & 1.00 \\
\hline
\end{tabular}



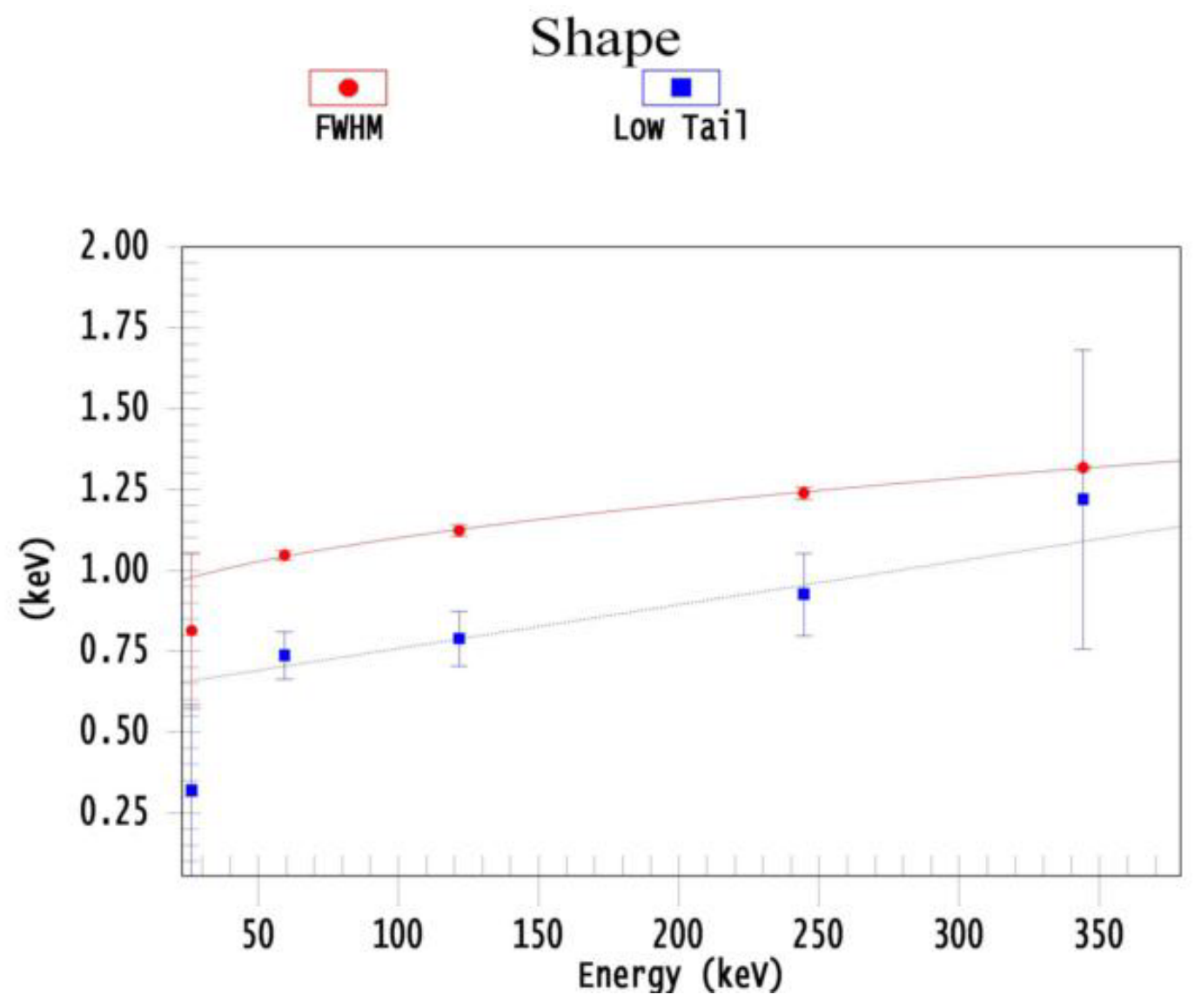

User: David Georgeson

Database: InVivo Server: 127.0.0.1 
Appendix D

\section{Efficiency Calibration Documentation}




\section{Appendix D \\ Efficiency Calibration Documentation}

Apex-InVivo

\section{Efficiency Calibration}

\section{Calibration Information}

\section{Calibration Title \\ Calibration Date \\ Performed By \\ Count Used \\ Approved \\ Is Default \\ Is Multi Curve}

\section{Counter Information}

\section{Counter Name}

Counter Location

Counter Configuration
Efficiency Calibration (I-125) 3/16/2011 4:31 PM

3/16/2011 4:29:50 PM

David Georgeson

Calibration Count 3/16/2011 12:56 PM

3/16/2011 4:31:59 PM by David Georgeson

Yes

No

Accuscan II

Battelle

Thyroid - I-125

\section{Detector Group Information}

Detector Group Name

Calibration Source

Analysis Sequence File

Nuclide Library

Peak Search Library
Summed

Am241/Eu152 RMC II-1

CALWBCGE for I-125.ASF

Am-241/Eu-152.NLB

Am-241/Eu-152.NLB

Approved by:

Date:

User: David Georgeson 
Efficiency Calibration

\section{Efficiency Triplets}

\begin{tabular}{|c|c|c|c|c|}
\hline Energy (keV) & Efficiency & Error & Dual & Difference \\
\hline $2.634 \mathrm{E}+001$ & $4.155 \mathrm{E}-003$ & $8.731 \mathrm{E}-005$ & 3.389E-003 & $18.43 \%$ \\
\hline $5.954 \mathrm{E}+001$ & $2.468 \mathrm{E}-003$ & $2.964 \mathrm{E}-005$ & $3.031 \mathrm{E}-003$ & $-22.83 \%$ \\
\hline $1.218 \mathrm{E}+002$ & $2.928 \mathrm{E}-003$ & $3.550 \mathrm{E}-005$ & $2.444 \mathrm{E}-003$ & $16.55 \%$ \\
\hline $2.447 \mathrm{E}+002$ & $1.883 \mathrm{E}-003$ & $4.280 \mathrm{E}-005$ & $1.782 \mathrm{E}-003$ & $5.38 \%$ \\
\hline $3.443 E+002$ & $1.377 \mathrm{E}-003$ & 1.824E-005 & $1.470 \mathrm{E}-003$ & $-6.78 \%$ \\
\hline
\end{tabular}

\section{Empirical}

$\ln ($ eff $)=-6.02946+0.756669 * x-0.579472 * x^{\wedge} 2-0.381448 * x^{\wedge} 3+0.283555 * x^{\wedge} 4$ where $x=\ln (185.311 / E)$

where $\mathrm{E}=$ Energy in keV

\section{Empirical}

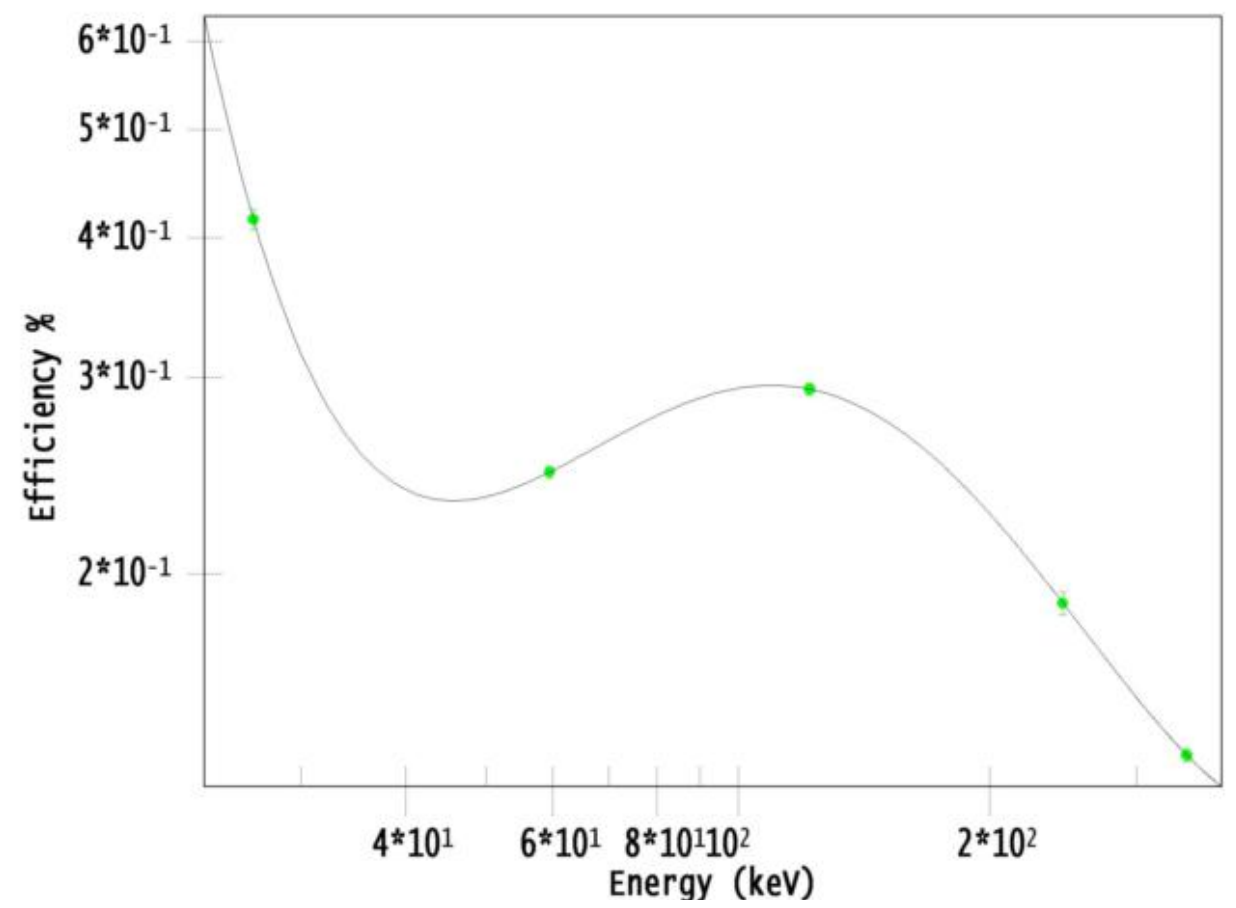

User: David Georgeson

Efficiency Calibration

\section{Dual}

$\ln ($ eff $)=-6.67176+0.651547 * \ln (E)-0.107164 *(\ln (E))^{\wedge} 2$

where $\mathrm{E}=$ Energy in keV

\section{Dual}

\section{$4 * 10-1$}


User: David Georgeson 
Efficiency Calibration

\section{Interpolated}

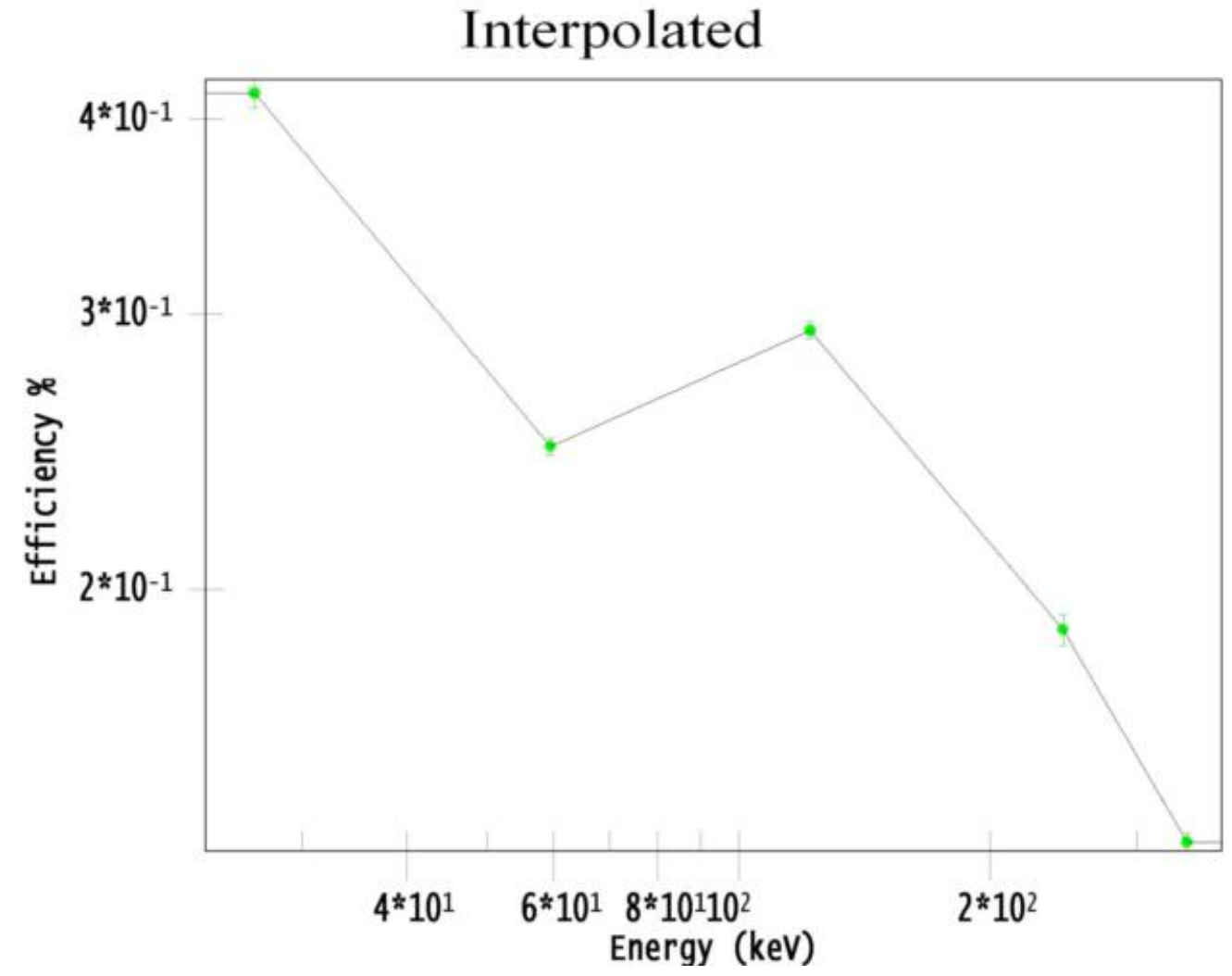

User: David Georgeson

Efficiency Calibration 


\section{Linear}

$\log ($ eff $)=-0.00100278 * E-2.50288+2.17845 / E$

where $\mathrm{E}=$ Energy in $\mathrm{keV}$

Linear

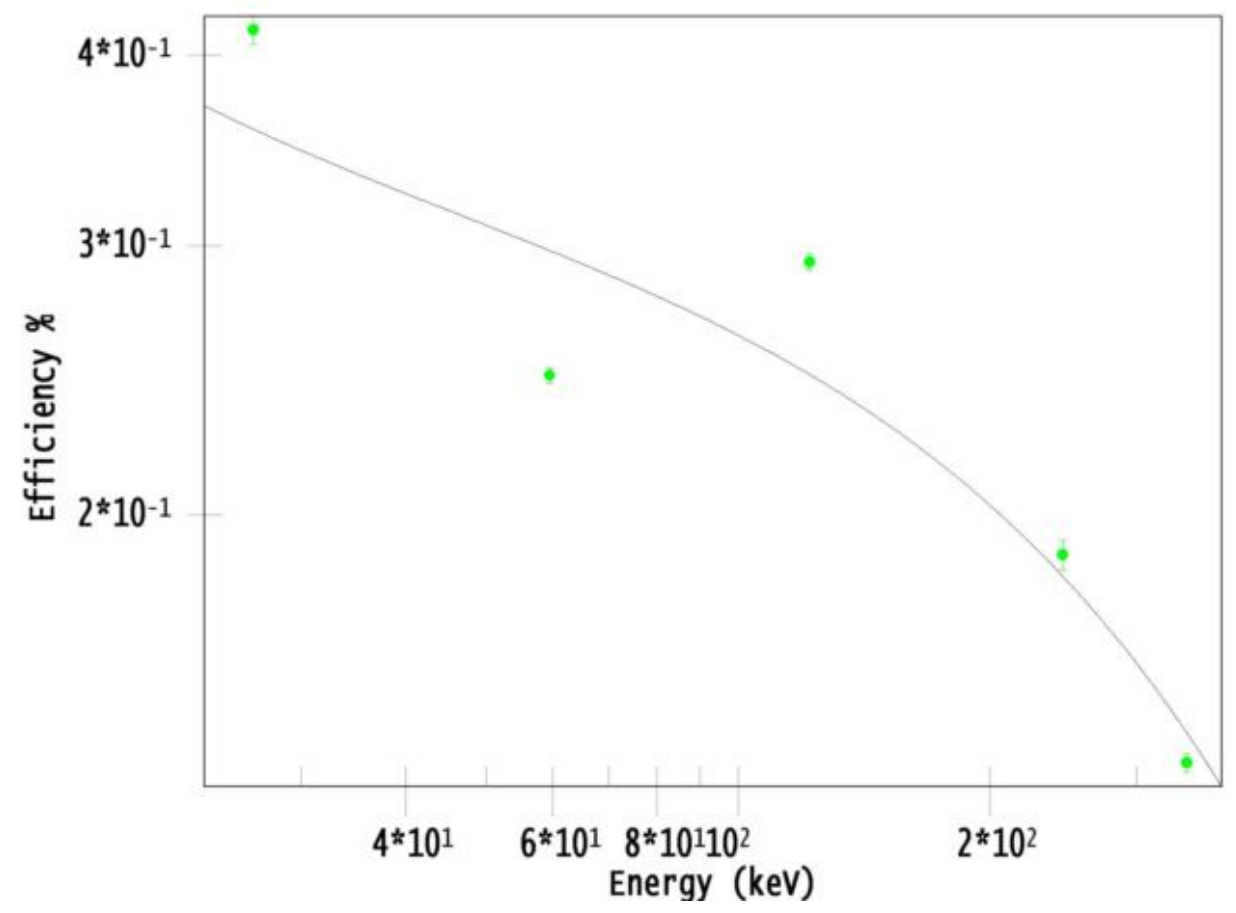

User: David Georgeson 
Appendix E

I-125 Calibration Count Reanalysis Summary 


\section{Appendix E \\ I-125 Calibration Count Reanalysis Summary}

Summary of the Reanalysis of the Efficiency Calibration Count For I-125 Calibration Using Am-241/Eu152

Arrangement/Geometry: Accuscan II

Analysis Library: Am-241/Eu-152 for I-125.NLB

Energy Calibration Time: 03/16/2011 3:02 PM

Efficiency Calibration Time: 03/16/2011 4:31 PM

Count Live Time: 7223 Seconds

Count Start Time: 3/16/2011 12:56 PM

\begin{tabular}{cccc}
$\begin{array}{c}\text { Nuclide/ } \\
\begin{array}{c}\text { Energy } \\
(\mathrm{keV})\end{array}\end{array}$ & $\begin{array}{c}\text { Expected } \\
\text { Activity } \\
(\mu \mathrm{Ci})\end{array}$ & $\begin{array}{c}\text { Observed } \\
\text { Activity } \\
(\mu \mathrm{Ci})\end{array}$ & $\begin{array}{c}\text { Relative } \\
\text { Bias }\end{array}$ \\
\hline Am-241/26.26 & 210 & 275.3 & 0.311 \\
Am-241/59.54 & 210 & 211.9 & 0.009 \\
Eu-152/121.8 & 180 & 180.400 & 0.002 \\
Eu-152/244.7 & 180 & 181.100 & 0.006 \\
Eu-152/344.3 & 180 & 178.200 & -0.010 \\
& & & \\
& & average & 0.064
\end{tabular}




\section{Analysis Report - Calibration Count 3/16/2011 12:56 PM}

\section{COUNTER INFORMATION}

Counter Name

Counter Location

Configuration Name
Accuscan II

Battelle

Thyroid - I-125

\section{COUNT INFORMATION}

$\begin{array}{ll}\begin{array}{l}\text { Count Operation } \\ \text { Comment } \\ \text { Intake Date }\end{array} & \text { Calibration Count } \\ \text { Acquisition Started } & \\ \text { Acquisition Time } & 3 / 16 / 2011 \quad 12: 56: 49 \text { PM } \\ \text { Operator Name } & 7223.15 \\ \text { Count Status } & \text { David Georgeson } \\ \text { Primary Review } & \text { Not Reviewed } \\ \text { Secondary Review } & \text { No } \\ \end{array}$

\section{DETECTOR COUNT RATE REPORT}

\begin{tabular}{|l|c|c|c|c|c|l|}
\hline \multicolumn{1}{|c|}{ Detector Name } & $\begin{array}{c}\text { Count } \\
\text { Rate }\end{array}$ & $\begin{array}{c}\text { Count } \\
\text { Rate }\end{array}$ & Live Time & $\begin{array}{c}\text { Analyze } \\
\text { d }\end{array}$ & Analyzed Date & File Name \\
\hline Left & 60.77 & $1-4096$ & 7200.0 & No & & b065b30a1256.cnf \\
\hline Right & 61.51 & $1-4096$ & 7200.0 & No & & 11 cc05321256.cnf \\
\hline Summed & 0.00 & $1-4096$ & 7200.0 & Yes & $4 / 27 / 2011$ 10:27:59 AM & 0d528c651256.cnf \\
\hline
\end{tabular}

Primary Review by:

Date:
Secondary Review by:

Date: 


\section{DETECTOR GROUP INFORMATION}

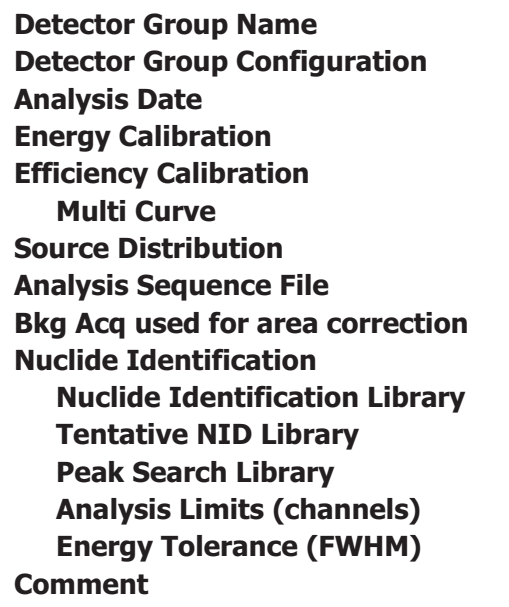

Summed

Thyroid Screening

4/27/2011 10:27:59 AM

Energy Calibration (I-125) 3/16/2011 3:02 PM

Efficiency Calibration (I-125) 3/16/2011 4:31 PM (Empirical)

No

Thyroid

Thyroid ASF for I-125.ASF

Not Performed

Am241/Eu152 for I-125

Am241/Eu152 for I-125

Am241/Eu152 for I-125

$20-4095$

1.20

PEAK SEARCH RESULTS FOR Accuscan II < Summed>

\begin{tabular}{|c|c|c|c|c|c|c|c|c|c|}
\hline \multicolumn{6}{|c|}{ Peak Analysis } & \multicolumn{4}{|c|}{ Nuclide Information } \\
\hline & No & \begin{tabular}{|l|} 
Peak \\
Centroid
\end{tabular} & $\begin{array}{l}\text { Energy } \\
(\mathrm{keV})\end{array}$ & \begin{tabular}{|l} 
Net Peak \\
Area
\end{tabular} & $\begin{array}{l}\text { Continuum } \\
\text { Counts }\end{array}$ & $\begin{array}{l}\text { Nuclide } \\
\text { Name }\end{array}$ & Activity (nCi) & $\begin{array}{l}\text { Error } \\
\text { (1SD) }\end{array}$ & $\begin{array}{l}\text { Yield } \\
(\%)\end{array}$ \\
\hline & 1 & 26.55 & 11.92 & $1.37 \mathrm{E}+003$ & $8.21 \mathrm{E}+003$ & Unknown & $5.788608 \mathrm{E}-003$ & & 100.00 \\
\hline M & 2 & 57.55 & 26.26 & 7.37E+003 & $7.18 \mathrm{E}+003$ & Am-241 & $2.752849 \mathrm{E}+002$ & $2.51 \%$ & 2.40 \\
\hline $\mathrm{m}$ & 3 & 65.54 & 29.95 & $4.19 E+003$ & $1.20 \mathrm{E}+004$ & Unknown & $4.971242 \mathrm{E}+000$ & & 100.00 \\
\hline $\mathrm{m}$ & 4 & 75.78 & 34.69 & $8.06 \mathrm{E}+003$ & $1.45 \mathrm{E}+004$ & Unknown & $1.158900 \mathrm{E}+001$ & & 100.00 \\
\hline $\mathrm{m}$ & 5 & 80.20 & 36.73 & $1.07 \mathrm{E}+004$ & $1.65 \mathrm{E}+004$ & Unknown & $1.607184 \mathrm{E}+001$ & & 100.00 \\
\hline $\mathrm{m}$ & 6 & 86.89 & 39.83 & $5.92 \mathrm{E}+004$ & $2.14 \mathrm{E}+004$ & Unknown & $9.303807 \mathrm{E}+001$ & & 100.00 \\
\hline M & 7 & 99.00 & 45.43 & $1.07 \mathrm{E}+004$ & $1.89 \mathrm{E}+004$ & Unknown & $1.733585 E+001$ & & 100.00 \\
\hline $\mathrm{m}$ & 8 & 107.88 & 49.54 & $5.45 E+002$ & 2.17E+004 & Unknown & 8.741777E-001 & & 100.00 \\
\hline & 9 & 129.50 & 59.54 & 4.97E+004 & $1.49 \mathrm{E}+004$ & Am-241 & $2.119438 \mathrm{E}+002$ & $1.43 \%$ & 35.70 \\
\hline & 10 & 264.08 & 121.77 & $4.00 \mathrm{E}+004$ & $9.77 \mathrm{E}+003$ & EU-152 & $1.804203 E+002$ & $1.44 \%$ & 28.40 \\
\hline & 11 & 530.03 & 244.71 & $6.81 E+003$ & $4.09 E+003$ & EU-152 & 1.811926E+002 & $3.58 \%$ & 7.49 \\
\hline & 12 & 640.53 & 295.77 & $3.66 \mathrm{E}+002$ & $2.31 \mathrm{E}+003$ & Unknown & $8.751176 \mathrm{E}-001$ & & 100.00 \\
\hline & 13 & 745.55 & 344.29 & $1.74 \mathrm{E}+004$ & $1.94 \mathrm{E}+003$ & EU-152 & $1.782479 \mathrm{E}+002$ & $1.68 \%$ & 26.60 \\
\hline & 14 & 796.32 & 367.74 & $3.29 \mathrm{E}+002$ & $1.65 \mathrm{E}+003$ & Unknown & $9.400238 \mathrm{E}-001$ & & 100.00 \\
\hline & 15 & 890.16 & 411.08 & $1.27 \mathrm{E}+003$ & $1.15 E+003$ & Unknown & $3.833098 \mathrm{E}+000$ & & 100.00 \\
\hline & 16 & 961.38 & 443.97 & $1.50 \mathrm{E}+003$ & $1.09 \mathrm{E}+003$ & Unknown & $4.647649 \mathrm{E}+000$ & & 100.00 \\
\hline & 17 & 1058.58 & 488.85 & $2.34 \mathrm{E}+002$ & $1.05 \mathrm{E}+003$ & Unknown & 7.219349E-001 & & 100.00 \\
\hline & 18 & 1106.81 & 511.12 & $7.33 \mathrm{E}+002$ & $1.08 \mathrm{E}+003$ & Unknown & $2.223503 E+000$ & & 100.00 \\
\hline & 19 & 1221.17 & 563.90 & $1.86 \mathrm{E}+002$ & $8.24 \mathrm{E}+002$ & Unknown & $5.279418 \mathrm{E}-001$ & & 100.00 \\
\hline & 20 & 1491.24 & 688.51 & $2.61 \mathrm{E}+002$ & $5.71 E+002$ & Unknown & 5.430381E-001 & & 100.00 \\
\hline & 21 & 1557.27 & 718.97 & $1.27 \mathrm{E}+002$ & $6.09 E+002$ & Unknown & 2.381721E-001 & & 100.00 \\
\hline & 22 & 1686.70 & 778.66 & $3.94 \mathrm{E}+003$ & $8.74 \mathrm{E}+002$ & Unknown & $5.839245 E+000$ & & 100.00 \\
\hline & 23 & 1878.37 & 867.02 & $1.16 \mathrm{E}+003$ & $7.36 \mathrm{E}+002$ & Unknown & $1.145140 \mathrm{E}+000$ & & 100.00 \\
\hline M & 24 & 1990.58 & 918.73 & $1.26 \mathrm{E}+002$ & $4.11 E+002$ & Unknown & $9.486010 \mathrm{E}-002$ & & 100.00 \\
\hline $\mathrm{m}$ & 25 & 2005.07 & 925.41 & $8.29 E+001$ & $4.16 \mathrm{E}+002$ & Unknown & 6.002627E-002 & & 100.00 \\
\hline
\end{tabular}




\begin{tabular}{|l|l|l|r|r|r||l|l|l|l|l|}
\hline & 26 & 2087.90 & 963.58 & $3.83 \mathrm{E}+003$ & $4.69 \mathrm{E}+002$ & Unknown & $2.239571 \mathrm{E}+000$ & & 100.00 \\
\hline & 27 & 2177.45 & 1004.84 & $1.38 \mathrm{E}+002$ & $3.31 \mathrm{E}+002$ & Unknown & $6.315312 \mathrm{E}-002$ & & 100.00 \\
\hline $\mathrm{M}$ & 28 & 2351.65 & 1085.07 & $2.39 \mathrm{E}+003$ & $3.56 \mathrm{E}+002$ & Unknown & $6.641177 \mathrm{E}-001$ & & 100.00 \\
\hline $\mathrm{m}$ & 29 & 2360.33 & 1089.07 & $3.33 \mathrm{E}+002$ & $4.61 \mathrm{E}+002$ & Unknown & $9.008405 \mathrm{E}-002$ & & 100.00 \\
\hline & 30 & 2408.52 & 1111.26 & $3.21 \mathrm{E}+003$ & $3.52 \mathrm{E}+002$ & Unknown & $7.509426 \mathrm{E}-001$ & & 100.00 \\
\hline & 31 & 2628.00 & 1212.29 & $2.69 \mathrm{E}+002$ & $3.15 \mathrm{E}+002$ & Unknown & $3.146647 \mathrm{E}-002$ & & 100.00 \\
\hline
\end{tabular}

User: David Georgeson

Database: InVivo Server: 127.0.0.1

Analysis Report - Calibration Count 3/16/2011 12:56 PM

Created 4/27/2011 10:28:10 AM Page 3 of 4

\section{PEAK SEARCH RESULTS FOR Accuscan II <Summed>}

\begin{tabular}{|c|c|c|c|c|c|c|c|c|c|}
\hline \multicolumn{6}{|c|}{ Peak Analysis } & \multicolumn{4}{|c|}{ Nuclide Information } \\
\hline & No & \begin{tabular}{|l} 
Peak \\
Centroid
\end{tabular} & $\begin{array}{l}\text { Energy } \\
(\mathrm{keV})\end{array}$ & $\begin{array}{l}\text { Net Peak } \\
\text { Area }\end{array}$ & $\begin{array}{l}\text { Continuum } \\
\text { Counts }\end{array}$ & $\begin{array}{l}\text { Nuclide } \\
\text { Name }\end{array}$ & Activity (nCi) & Error (1SD) & $\begin{array}{l}\text { Yield } \\
(\%)\end{array}$ \\
\hline & 32 & 3052.74 & 1407.70 & $4.05 E+003$ & $1.32 \mathrm{E}+002$ & Unknown & $1.092345 \mathrm{E}-$ & & 100.00 \\
\hline M & 33 & 3161.55 & 1457.74 & $3.17 \mathrm{E}+002$ & $7.80 \mathrm{E}+001$ & Unknown & $5.747485 \mathrm{E}-$ & & 100.00 \\
\hline $\mathrm{m}$ & 34 & 3167.10 & 1460.29 & $3.50 \mathrm{E}+002$ & $7.65 \mathrm{E}+001$ & Unknown & $6.232102 \mathrm{E}-$ & & 100.00 \\
\hline
\end{tabular}

For unknown peaks we assume yield to be $100 \%$ and no decay correction is performed.

$M=$ First peak in a multiplet region

$\mathrm{m}=$ Other peak in a multiplet region

$\mathrm{F}=$ Fitted singlet

NUCLIDE RESULTS FOR Accuscan II <Summed>

\begin{tabular}{|l|l|l|l|r|l|l|l|}
\hline & $\begin{array}{l}\text { Nuclide } \\
\text { Name }\end{array}$ & $\begin{array}{l}\text { Id } \\
\text { Confidence }\end{array}$ & $\begin{array}{l}\text { Wt Mean } \\
\text { Activity (nCi) }\end{array}$ & Error (1SD) & Action Level 1 & Action Level 2 & MDA (nCi) \\
\hline & EU-152 & 1.000 & $1.796259 \mathrm{E}+00$ & $1.05 \%$ & \multirow{2}{*}{ Not Performed } & Not Performed & $2.545 \mathrm{E}+000$ \\
\cline { 2 - 5 } & Am-241 & 1.000 & $2.221031 \mathrm{E}+00$ & $1.25 \%$ & & & $3.074 \mathrm{E}+000$ \\
\hline
\end{tabular}

$?=$ Nuclide is part of an undetermined solution

$\mathrm{X}=$ Nuclide rejected by the interference analysis

@ = Nuclide contains energy lines not used in Weighted Mean Activity

! = Nuclide was corrected for parent/daughter

NUCLIDE MDA RESULTS FOR Accuscan II <Summed>

\begin{tabular}{|c|c|c|c|c|c|c|}
\hline & Nuclide & Energy (keV) & Yield (\%) & Line MDA & Nuclide MDA & Activity (nCi) \\
\hline+ & \multirow[t]{3}{*}{ EU-152 } & 121.78 * & 28.40 & $2.5446 \mathrm{E}+000$ & \multirow{3}{*}{$2.5446 \mathrm{E}+000$} & $1.8042 \mathrm{E}+002$ \\
\hline+ & & 244.70 * & 7.49 & $1.0491 \mathrm{E}+001$ & & $1.8119 \mathrm{E}+002$ \\
\hline+ & & 344.28 * & 26.60 & $2.8828 E+000$ & & $1.7825 \mathrm{E}+002$ \\
\hline+ & \multirow[t]{2}{*}{ Am-241 } & 26.30 * & 2.40 & $1.0422 \mathrm{E}+001$ & \multirow{2}{*}{$3.0736 \mathrm{E}+000$} & $2.7528 \mathrm{E}+002$ \\
\hline+ & & 59.54 * & 35.70 & $3.0736 \mathrm{E}+000$ & & $2.1194 \mathrm{E}+002$ \\
\hline
\end{tabular}

$+=$ Nuclide Identified during the nuclide identification

* = Energy Line found in the spectrum 


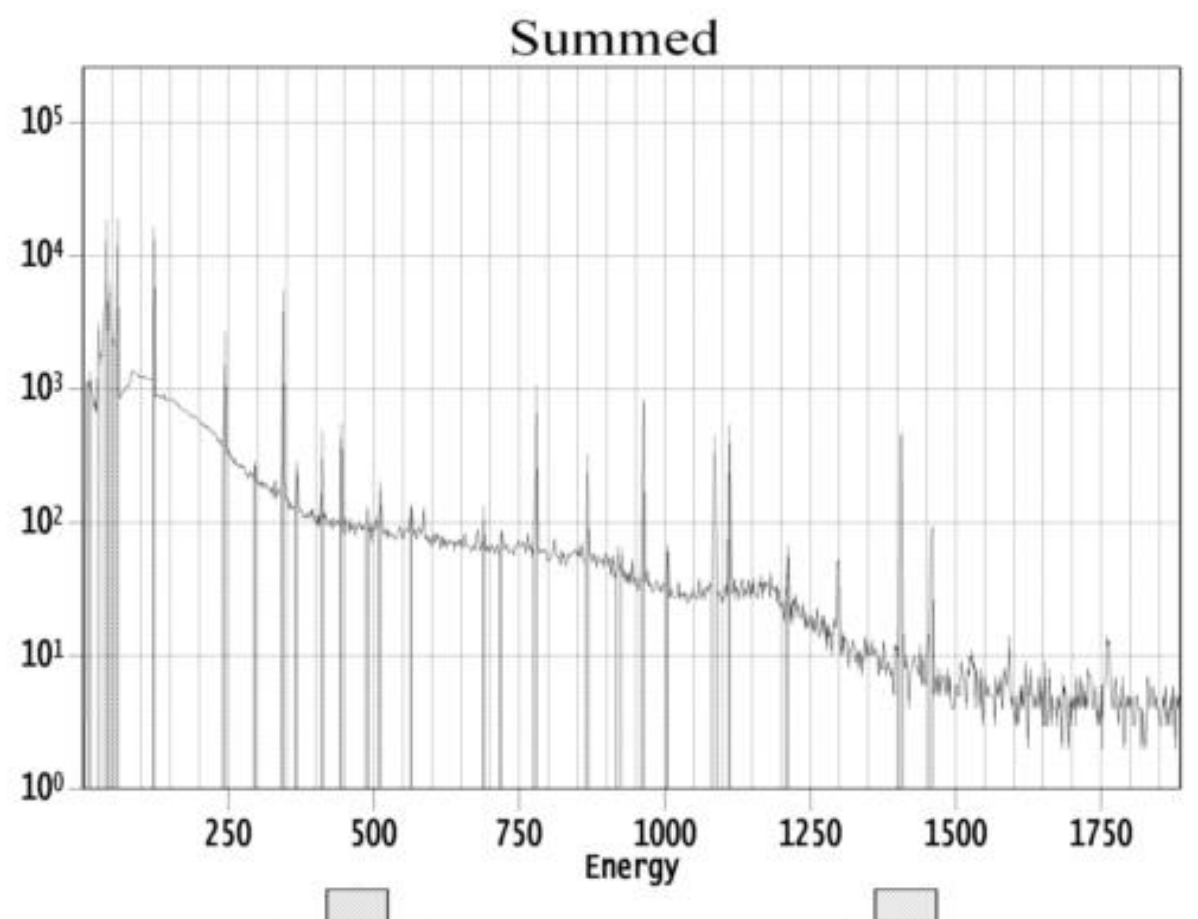

ROI Type: 1

ROI Type: 2 


\section{Appendix F}

\section{Accuscan II I-125 Calibration Verification}




\section{Appendix $\mathbf{F}$ \\ Accuscan II I-125 Calibration Verification}

Apex-InVivo

Page 1 of 2

Created 4/26/2011 5:22:10 PM

Idaho Falls Id.

Battelle Energy Alliance LLC

\section{Efficiency Verification}

\section{Calibration Information}

Calibration Title

Calibration Date

Operator Name

Detector Group

Detector Group

Source Distribution

\author{
Efficiency Calibration (I-125) 3/16/2011 4:31 PM \\ 3/16/2011 4:29:50 PM \\ David Georgeson \\ Summed \\ Thyroid Screening \\ Thyroid
}

\section{Counter Information}

$\begin{array}{ll}\text { Counter Name } & \text { Accuscan II } \\ \text { Counter Location } & \text { Battelle } \\ \text { Counter } & \text { Thyroid - I-125 }\end{array}$

\section{Count List}

\section{Num Date}

$13 / 16 / 2011$

$23 / 16 / 2011$

$3 \quad 3 / 16 / 2011$

$4 \quad 3 / 16 / 2011$

$53 / 16 / 2011$

\section{Name}

Verification Count 3/16/2011 3:13 PM (1/5)

Verification Count 3/16/2011 3:13 PM (2/5)

Verification Count 3/16/2011 3:13 PM (3/5)

Verification Count 3/16/2011 3:13 PM (4/5)

Verification Count 3/16/2011 3:13 PM (5/5) 


\begin{tabular}{|c|c|c|c|c|c|c|c|c|c|c|}
\hline User: Davi & 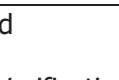 & & & & & & & & & Database \\
\hline Efficiency & Verificatio & & & & & & & Created & $4 / 26 / 2011$ & 22:10 PM Pag \\
\hline Source C & ertificate & Am241/ & U152 RMC I & & & & & & & \\
\hline Nuclide & Energy & $\begin{array}{c}\text { Certificate } \\
\text { Activity } \\
(\mathrm{g} / \mathrm{s})\end{array}$ & Half Life & Count 1 & Count 2 & Count 3 & Count 4 & Count 5 & $\begin{array}{l}\text { Relative } \\
\text { Bias (\%) }\end{array}$ & $\begin{array}{c}\text { Relative } \\
\text { Precision } \\
(\%)\end{array}$ \\
\hline$\Delta m-241$ & 1303 & 1900 ח & $43210 \times$ & 0.00 & 0.00 & 0.00 & 0.00 & 0.00 & - & - \\
\hline HIII-Z41 & נכ.ר. & 1900.00 & $452.19 y$ & 0.00 & 0.00 & 0.00 & 0.00 & 0.00 & - & \\
\hline Am-241 & 1751 & 237000 & $43219 v$ & 0.00 & 0.00 & 0.00 & 0.00 & 0.00 & - & - \\
\hline AIII-Z41 & $1 / .51$ & 2570.00 & $432.19 y$ & 0.00 & 0.00 & 0.00 & 0.00 & 0.00 & & \\
\hline Am-241 & 20.98 & 568.00 & $43219 \mathrm{v}$ & 0.00 & 0.00 & 0.00 & 0.00 & 0.00 & - & - \\
\hline & & & & 0.00 & 0.00 & 0.00 & 0.00 & 0.00 & & \\
\hline Am-241 & 26.34 & 186.00 & $43219 v$ & 0.00 & 163.88 & 165.68 & 189.54 & 0.00 & $-697 \%$ & $629 \%$ \\
\hline AIII-Z4I & 20.34 & 100.00 & & 0.00 & 186.00 & 186.00 & 186.00 & 0.00 & $-6.97 \%$ & $0.29 \%$ \\
\hline Am-241 & 59.54 & 277000 & $43219 \mathrm{v}$ & 2787.36 & 2734.35 & 2786.32 & 2727.81 & 3065.74 & $182 \%$ & $452 \%$ \\
\hline & & & & 2769.97 & 2769.97 & 2769.97 & 2769.97 & 2769.97 & & \\
\hline EU-152 & 121.78 & 1890.00 & $494621 d$ & 1796.42 & 1991.97 & 1855.09 & 1938.08 & 1902.02 & $039 \%$ & $356 \%$ \\
\hline & & & & 1889.30 & 1889.30 & 1889.30 & 1889.30 & 1889.30 & & \\
\hline FH-152 & 24470 & $500 \Omega 0$ & dיכ 4946 & 421.53 & 443.33 & 471.88 & 570.06 & 573.26 & $-076 \%$ & $1277 \%$ \\
\hline$[0-132$ & 244.70 & 500.00 & $4940.22 \mathrm{~d}$ & 499.82 & 499.81 & 499.81 & 499.81 & 499.81 & $-0.10 \%$ & $12.17 \%$ \\
\hline EU-152 & 344.28 & 1770.00 & $494622 d$ & 1783.19 & 1770.16 & 1635.56 & 1851.77 & 1785.85 & $-0.23 \%$ & $400 \%$ \\
\hline$[U-152$ & 344.28 & $17 / 0.00$ & $4946.22 \mathrm{a}$ & 1769.35 & 1769.34 & 1769.34 & 1769.34 & 1769.34 & $-0 . \angle 3 \%$ & $4.00 \%$ \\
\hline & & & & & & & & $|x b a r|=$ & $2.03 \%$ & $6.23 \%$ \\
\hline
\end{tabular}

For each count, the number on the top is the observed activity and the number on the bottom is the expected activity.

References:

Draft American National Standard, Performance Criteria for Radiobioassay, ANSI N13.30

Certificate of Calibration, Standard Radionuclide Source, Analytics 
Appendix G

\section{MDA Determination}




\title{
Appendix G \\ MDA Determination \\ MDA SUMMARY CALCULATIONS FROM COUNT DATA
}

\author{
MDA CALCULATION For I-125 \\ ACCUSCAN II COUNTER
}

MDA Calculations

Arrangement Geometry: Thyroid

Analysis Library: I-125 Thyroid.nlb

Energy Calibration Time: 3/16/2011 3:02 PM

Efficiency Calibration Time: 3/16/2011 4:31 PM

Count Type/Live Time: 600 Seconds

Count Start Times:

1. $3 / 23 / 114: 19 \mathrm{PM}$

2. $3 / 23 / 114: 32 \mathrm{PM}$

3. $3 / 23 / 114: 44 \mathrm{PM}$

$\mathrm{MDA}=(4.65 \mathrm{Sb}+3) / \mathrm{KT}$

Where:

$\mathrm{Sb}=$ Standard deviation of the blank phantom counts $=$ SQRT

(gross rate (CPS) $x$ routine counting time)

$\mathrm{K}=$ Efficiency $X$ Abundance $X 37 \mathrm{~Bq} / \mathrm{nCi}$

$\mathrm{T}=$ Routine counting time in seconds $=600$ seconds

Nuclide/Energy \#1 Count (MDA) \#2 Count (MDA) \#3 Count (MDA) Average

\begin{tabular}{lcccc} 
& $(\mathrm{nCi})$ & $(\mathrm{nCi})$ & $(\mathrm{nCi})$ & $(\mathrm{nCi})$ \\
\hline & & & & \\
$\mathrm{I}-125 / 27 \mathrm{keV}$ & $1.99 \mathrm{E}+00$ & $2.03 \mathrm{E}+00$ & $3.10 \mathrm{E}+00$ & $2.57 \mathrm{E}+00$ \\
$\mathrm{l}-125 / 31 \mathrm{keV}$ & $6.01 \mathrm{E}+00$ & $7.35 \mathrm{E}+00$ & $6.59 \mathrm{E}+00$ & $6.97 \mathrm{E}+00$
\end{tabular}




\section{Analysis Report - I-125 Validation Count [000-125]}

\section{SOURCE INFORMATION}

$\begin{array}{ll}\text { Primary ID } & 000-125 \\ \text { Last Name } & \text { Validation Count } \\ \text { First Name } & \text { I-125 } \\ \text { Height (in) } & \\ \text { Weight (Ib) } & \\ \text { Chest Wall Thickness (cm) } & \end{array}$

\section{COUNTER INFORMATION}

Counter Name

Counter Location

Configuration Name
Accuscan II

Battelle

Thyroid - I-125

\section{COUNT INFORMATION}

$\begin{array}{ll}\begin{array}{l}\text { Count Operation } \\ \text { Count Reason } \\ \text { Frequency } \\ \text { Comment }\end{array} & \begin{array}{l}\text { Individual } 10 \text { minutes } \\ \text { Routine operation }\end{array} \\ \text { Intake Date } & \\ \text { Acquisition Started } & \\ \text { Acquisition Time } & 3 / 23 / 20114: 19: 49 \text { PM } \\ \text { Operator Name } & 601.24 \\ \text { Count Status } & \text { David Georgeson } \\ \text { Primary Review } & \text { Not Reviewed } \\ \text { Secondary Review } & \text { No } \\ \end{array}$

\section{DETECTOR COUNT RATE REPORT}

\begin{tabular}{|l|r|r|r|c|}
\hline \multicolumn{1}{|c|}{ Detector Name } & $\begin{array}{c}\text { Count } \\
\text { Rate }\end{array}$ & $\begin{array}{c}\text { Count Rate } \\
\text { Range }\end{array}$ & Live Time & Analyzed \\
\hline Left & 13.30 & $1-4096$ & 600.0 & No \\
\hline Right & 14.40 & $1-4096$ & 600.0 & No \\
\hline Summed & 27.65 & $1-4096$ & 600.0 & Yes \\
\hline
\end{tabular}

Primary Review by:

Date: 


\section{DETECTOR GROUP INFORMATION}

Detector Group Name

Detector Group Configuration

Analysis Date

Energy Calibration

Efficiency Calibration

Multi Curve

Source Distribution

Analysis Sequence File

Bkg Acq used for area correction

Nuclide Identification

Nuclide Identification Library

Tentative NID Library

Peak Search Library

Analysis Limits (channels)

Energy Tolerance (FWHM)

Comment
Summed

Thyroid Screening

3/23/2011 4:19:39 PM

Energy Calibration (I-125) 3/16/2011 3:02 PM

Efficiency Calibration (I-125) 3/16/2011 4:31 PM (Empirical)

No

Thyroid

Thyroid ASF for I-125.ASF

Not Performed

I-125 Thyroid.nlb

I-125 Thyroid.nlb

I-125 Thyroid.nlb

$20-4095$

1.20

NUCLIDE MDA RESULTS FOR Accuscan II <Summed>

\begin{tabular}{|c|c|c|c|}
\hline \begin{tabular}{|l} 
Nuclide \\
Name
\end{tabular} & Energy (keV) & Yield (\%) & Line MDA (nCi) \\
\hline \multirow[t]{2}{*}{ I-125 } & 27.00 & 40.25 & $1.9877 \mathrm{E}+000$ \\
\hline & 31.00 & 12.50 & $6.0148 \mathrm{E}+000$ \\
\hline
\end{tabular}

* = Energy Line found in the spectrum

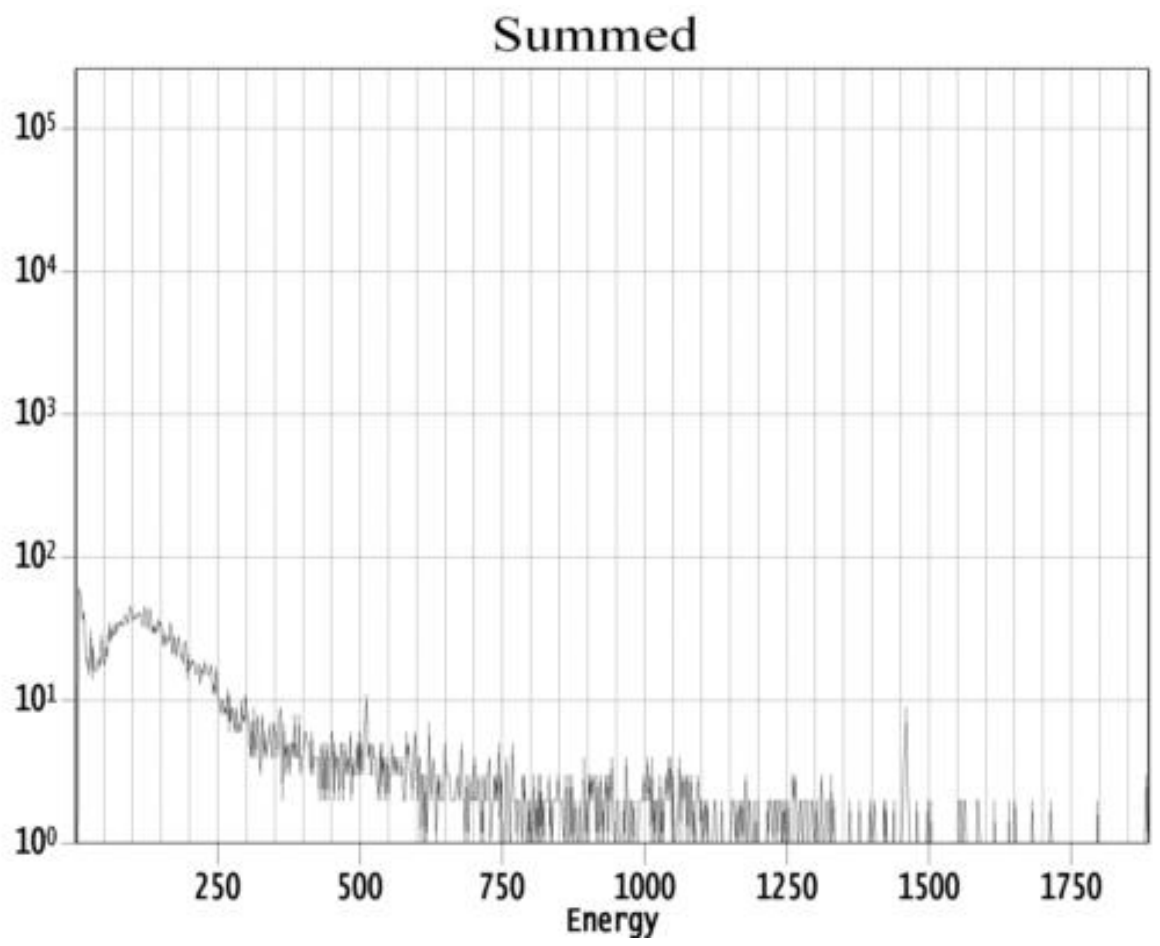

User: David Georgeson 


\section{Analysis Report - I-125 Validation Count [000-125]}

\section{SOURCE INFORMATION}

$\begin{array}{ll}\text { Primary ID } & 000-125 \\ \text { Last Name } & \text { Validation Count } \\ \text { First Name } & \text { I-125 } \\ \text { Height (in) } & \\ \text { Weight (Ib) } & \\ \text { Chest Wall Thickness (cm) } & \end{array}$

\section{COUNTER INFORMATION}

Counter Name

Counter Location

Configuration Name
Accuscan II

Battelle

Thyroid - I-125

\section{COUNT INFORMATION}

$\begin{array}{ll}\begin{array}{l}\text { Count Operation } \\ \text { Count Reason }\end{array} & \begin{array}{l}\text { Individual } 10 \text { minutes } \\ \text { Routine operation }\end{array} \\ \begin{array}{l}\text { Frequency } \\ \text { Comment }\end{array} & \\ \text { Intake Date } & \\ \text { Acquisition Started } & \\ \text { Acquisition Time } & 3 / 23 / 20114: 32: 40 \text { PM } \\ \text { Operator Name } & 601.35 \\ \text { Count Status } & \text { David Georgeson } \\ \text { Primary Review } & \text { Not Reviewed } \\ \text { Secondary Review } & \text { No } \\ \end{array}$

\section{DETECTOR COUNT RATE REPORT}

\begin{tabular}{|l|r|r|r|c|}
\hline \multicolumn{1}{|c|}{ Detector Name } & Count Rate & $\begin{array}{c}\text { Count Rate } \\
\text { Range }\end{array}$ & Live Time & $\begin{array}{c}\text { Analyze } \\
\text { d }\end{array}$ \\
\hline Right & 14.62 & $1-4096$ & 600.0 & No \\
\hline Left & 13.44 & $1-4096$ & 600.0 & No \\
\hline Summed & 27.98 & $1-4096$ & 600.0 & Yes \\
\hline
\end{tabular}

Primary Review by:

Date:

User: David Georgeson

Analysis Report - I-125 Validation Count [000-125] 


\section{DETECTOR GROUP INFORMATION}

\section{Detector Group Name \\ Detector Group Configuration \\ Analysis Date \\ Energy Calibration \\ Efficiency Calibration \\ Multi Curve}

Source Distribution

Analysis Sequence File

Bkg Acq used for area correction

Nuclide Identification

Nuclide Identification Library

Tentative NID Library

Peak Search Library

Analysis Limits (channels)

Energy Tolerance (FWHM)
Summed

Thyroid Screening

3/23/2011 4:32:13 PM

Energy Calibration (I-125) 3/16/2011 3:02 PM

Efficiency Calibration (I-125) 3/16/2011 4:31 PM (Empirical)

No

Thyroid

Thyroid ASF for I-125.ASF

Not Performed

I-125 Thyroid.nlb

I-125 Thyroid.nlb

I-125 Thyroid.nlb

$20-4095$

1.20

\section{Comment}

NUCLIDE MDA RESULTS FOR Accuscan II <Summed>

\begin{tabular}{|l|l|r|r|l|}
\hline & $\begin{array}{l}\text { Nuclide } \\
\text { Name }\end{array}$ & Energy (keV) & Yield (\%) & $\begin{array}{l}\text { Line MDA } \\
\text { (nCi) }\end{array}$ \\
\hline \multirow{2yyy}{*}{ I-125 } & 27.00 & 40.25 & $2.0337 \mathrm{E}+000$ \\
\cline { 3 - 5 } & 31.00 & 12.50 & $7.3530 \mathrm{E}+000$ \\
\hline
\end{tabular}

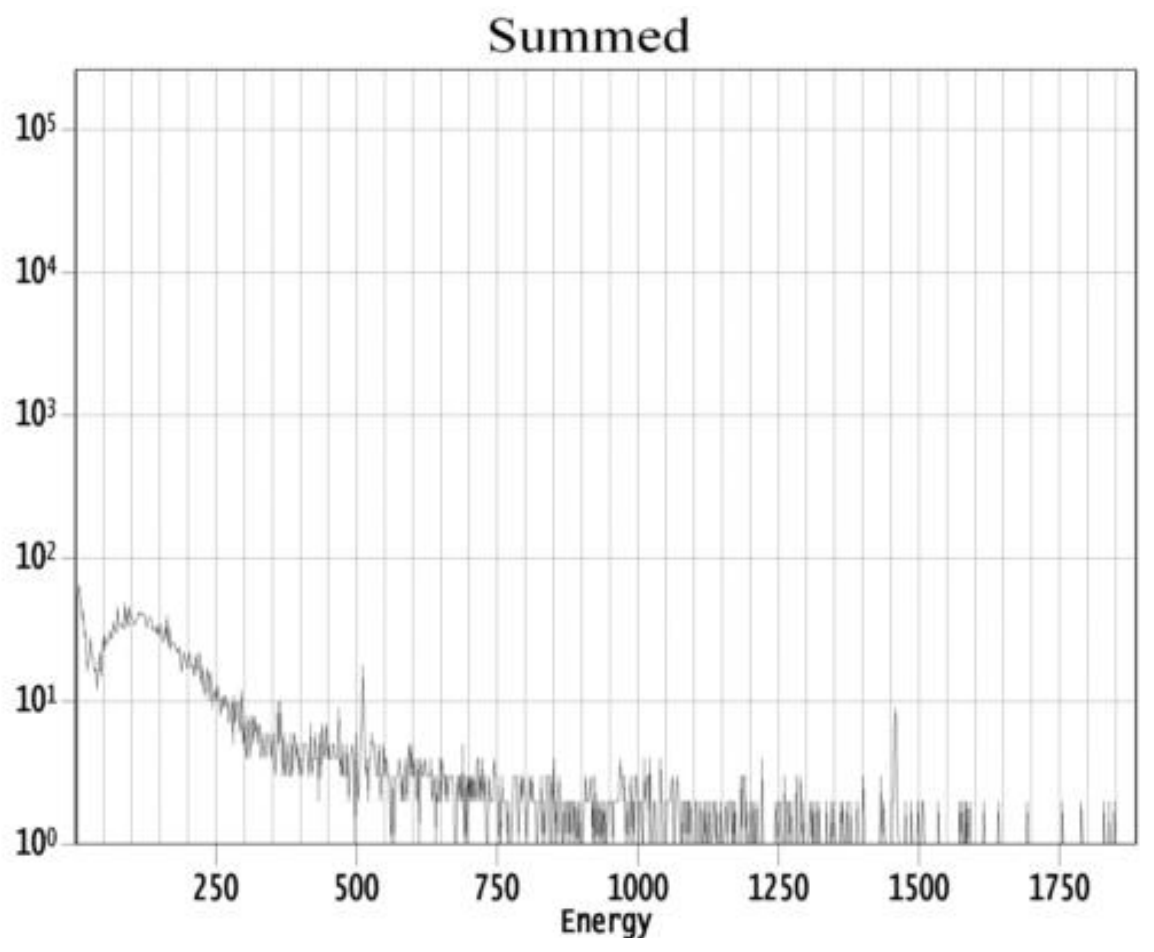

User: David Georgeson 


\section{Analysis Report - I-125 Validation Count [000-125]}

\section{SOURCE INFORMATION}

Primary ID

Last Name

First Name

Height (in)

Weight (lb)

Chest Wall Thickness (cm)
000-125

Validation Count

I-125

\section{COUNTER INFORMATION}

Counter Name

Counter Location

Configuration Name
Accuscan II

Battelle

Thyroid - I-125

\section{COUNT INFORMATION}

\section{Count Operation}

Count Reason

Frequency

Comment

Intake Date

Acquisition Started

Acquisition Time

Operator Name

Count Status

Primary Review

Secondary Review
Individual 10 minutes

Routine operation

3/23/2011 4:44:28 PM
601.4299999
David Georgeson
Not Reviewed
No
No

3/23/2011 4:44:28 PM

601.4299999

Not Reviewed

No

\section{DETECTOR COUNT RATE REPORT}

\begin{tabular}{|l|r|r|c|c|}
\hline \multicolumn{1}{|c|}{ Detector Name } & Count Rate & $\begin{array}{c}\text { Count Rate } \\
\text { Range }\end{array}$ & $\begin{array}{c}\text { Live } \\
\text { Time }\end{array}$ & Analyzed \\
\hline Left & 13.44 & $1-4096$ & 600.0 & No \\
\hline Right & 14.72 & $1-4096$ & 600.0 & No \\
\hline Summed & 28.11 & $1-4096$ & 600.0 & Yes \\
\hline
\end{tabular}

Primary Review by:

Date: 


\section{DETECTOR GROUP INFORMATION}

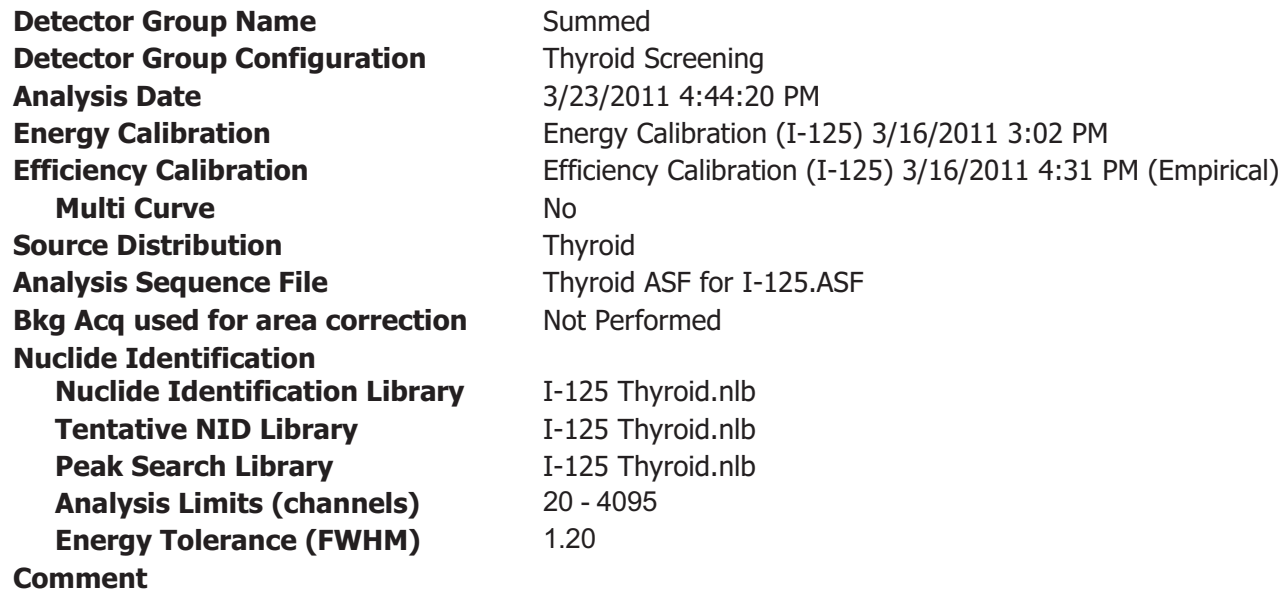

Summed

Thyroid Screening

3/23/2011 4:44:20 PM

Energy Calibration (I-125) 3/16/2011 3:02 PM

Efficiency Calibration (I-125) 3/16/2011 4:31 PM (Empirical)

No

Thyroid

Thyroid ASF for I-125.ASF

Not Performed

I-125 Thyroid.nlb

I-125 Thyroid.nlb

I-125 Thyroid.nlb

$20-4095$

1.20

PEAK SEARCH RESULTS FOR Accuscan II <Summed>

\begin{tabular}{|r|r|l|l|l|l||}
\hline \multicolumn{7}{|c|}{ Peak Analysis } \\
\hline \multirow{2}{*}{ No } & $\begin{array}{l}\text { Peak } \\
\text { Centroid }\end{array}$ & $\begin{array}{l}\text { Energy } \\
\text { (keV) }\end{array}$ & $\begin{array}{l}\text { Net Peak } \\
\text { Area }\end{array}$ & $\begin{array}{l}\text { Continuum } \\
\text { Counts }\end{array}$ \\
\hline & 1 & 57.75 & 26.35 & $7.98 \mathrm{E}+001$ & $1.06 \mathrm{E}+002$ \\
\hline
\end{tabular}

For unknown peaks we assume yield to be $100 \%$ and no decay correction is performed

$\mathrm{M}=$ First peak in a multiplet region

$\mathrm{m}=$ Other peak in a multiplet region

$\mathrm{F}=$ Fitted singlet

NUCLIDE RESULTS FOR Accuscan II <Summed>

\begin{tabular}{|l|l|l|l|l|}
\hline & $\begin{array}{l}\text { Nuclide } \\
\text { Name }\end{array}$ & $\begin{array}{l}\text { Id } \\
\text { Confidence }\end{array}$ & $\begin{array}{l}\text { Wt Mean Activity } \\
\text { (nCi) }\end{array}$ & Error (1SD) \\
\hline $\mathrm{I}-125$ & 0.987 & $2.152364 \mathrm{E}+000$ & $32.42 \%$ \\
\hline
\end{tabular}

$?=$ Nuclide is part of an undetermined solution

$X=$ Nuclide rejected by the interference analysis

@ = Nuclide contains energy lines not used in Weighted Mean Activity

! = Nuclide was corrected for parent/daughter

\section{NUCLIDE MDA RESULTS FOR Accuscan II <Summed>}

\begin{tabular}{|c|l|r|r|r|}
\hline & $\begin{array}{l}\text { Nuclide } \\
\text { Name }\end{array}$ & Energy (keV) & \multicolumn{1}{|l|}{ Yield (\%) } & Line MDA (nCi) \\
\hline+ & $\mathrm{I}-125$ & $27.00 *$ & 40.25 & $3.1088 \mathrm{E}+000$ \\
\hline+ & & 31.00 & 12.50 & $6.5948 \mathrm{E}+000$ \\
\cline { 2 - 4 } & &
\end{tabular}

$+=$ Nuclide Identified during the nuclide identification

$*$ = Energy Line found in the spectrum 


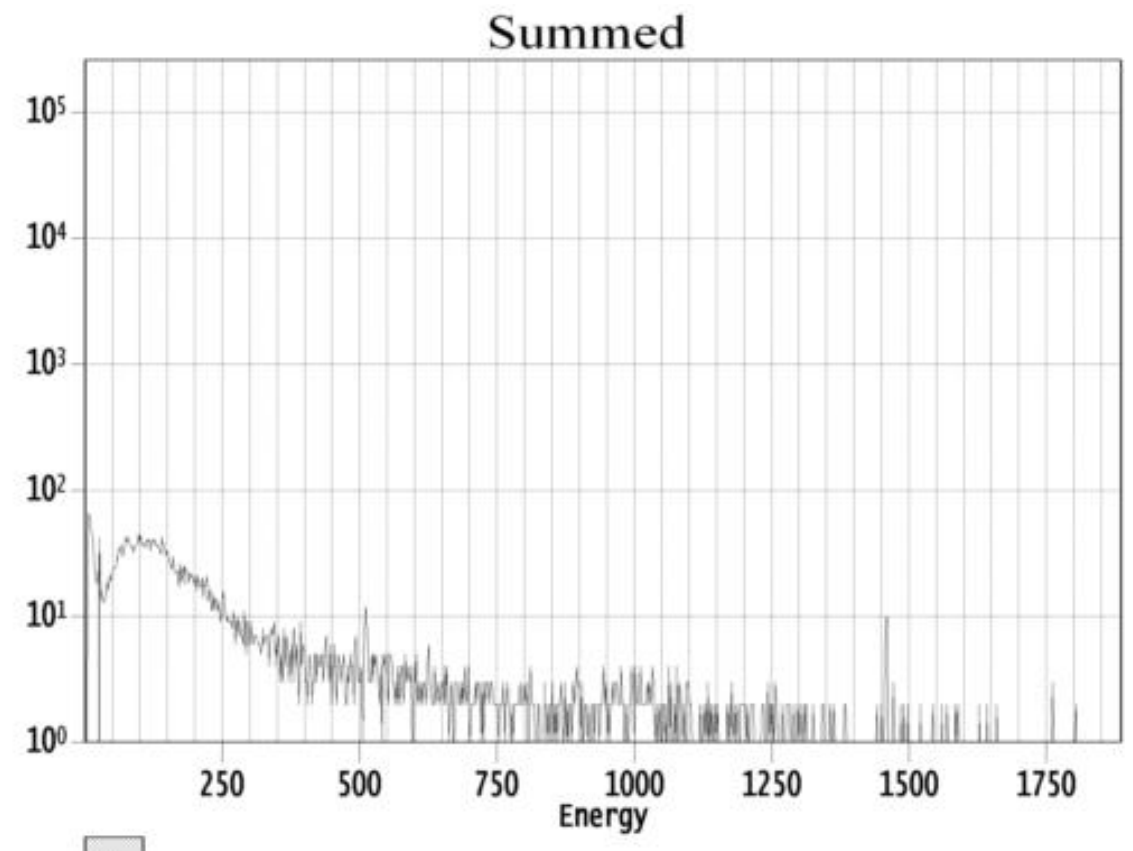

ROI Type: 1 


\section{Appendix $\mathrm{H}$}

\section{Calibration Validation using I-125}




\section{Appendix $\mathrm{H}$ \\ Calibration Validation using I-125 \\ Summary}

Calibration Validation Counts

Accuscan II Calibration Validation for I-125 Thyroid

Arrangement/Geometry: Thyroid

Analysis Library: I-125 Thyroid.nlb

Energy Calibration Date: 3/16/2011 3:02 PM

Efficiency Calibration Date: 3/16/2011 4:31 P

Count Live Time: 600 Seconds

Count Start Times:

Count \# 1 - 03/23/2011 3:09 PM

Count \# 2 - 03/23/2011 3:25 PM

Count \# 3 - 03/23/2011 3:41 PM

Count \# 4 - 03/23/2011 3:53 PM

Count \# 5 - 03/23/2011 4:05 PM

\begin{tabular}{ccccccccc}
$\begin{array}{c}\text { Nuclide/ } \\
\text { Energy } \\
(\mathrm{keV})\end{array}$ & $\begin{array}{c}\text { Expected } \\
\text { Activity } \\
(\mathrm{nCi})\end{array}$ & $\# 1$ & $\# 2$ & $\# 3$ & $\# 4$ & $\# 5$ & $\begin{array}{c}\text { Relative } \\
\text { Bias }\end{array}$ & $\begin{array}{c}\text { Relative } \\
\text { Precision }\end{array}$ \\
\hline $\mathrm{I}-125 / 27$ & 102.0000 & 98.600 & 97.800 & 95.600 & 97.300 & 96.700 & -0.047 & 0.011 \\
$\mathrm{I}-125 / 31$ & 102.0000 & 102.600 & 105.100 & 101.900 & 98.000 & 86.200 & -0.032 & 0.073
\end{tabular}

Average $\quad-0.040 \quad 0.042$ 


\section{COUNT 1}

\section{Apex-InVivo}

\section{Analysis Report - I-125 Validation Count [000-125]}

\section{SOURCE INFORMATION}

Primary ID

Last Name

First Name

Height (in)

Weight (Ib)

Chest Wall Thickness (cm)
000-125

Validation Count

I-125

\section{COUNTER INFORMATION}

\section{Counter Name \\ Counter Location \\ Configuration Name}

Accuscan II

Battelle

Thyroid - I-125

\section{COUNT INFORMATION}

\begin{tabular}{ll}
$\begin{array}{l}\text { Count Operation } \\
\text { Count Reason } \\
\text { Frequency } \\
\text { Comment } \\
\text { Intake Date }\end{array}$ & $\begin{array}{l}\text { Individual } 10 \text { minutes } \\
\text { Routine operation }\end{array}$ \\
Acquisition Started & \\
Acquisition Time & \\
Operator Name & $3 / 23 / 20113: 09: 07$ PM \\
Count Status & 601.5 \\
Primary Review & David Georgeson \\
Secondary Review & Not Reviewed \\
\hline
\end{tabular}

DETECTOR COUNT RATE REPORT

\begin{tabular}{|l|c|c|c|c|c|l|}
\hline \multicolumn{1}{|c|}{ Detector Name } & $\begin{array}{c}\text { Count } \\
\text { Rate }\end{array}$ & $\begin{array}{c}\text { Count } \\
\text { Rate }\end{array}$ & Live Time & Analyzed & Analyzed Date & File Name \\
\hline Left & 18.69 & $1-4096$ & 600.0 & No & & 9dab9d661508.cnf \\
\hline Right & 20.50 & $1-4096$ & 600.0 & No & & 0cbf77fc1508.cnf \\
\hline Summed & 39.07 & $1-4096$ & 600.0 & Yes & $3 / 23 / 20113: 08: 58$ PM & 03ddb8681508.cnf \\
\hline
\end{tabular}

Primary Review by:

Date:

\section{Secondary Review}

Date: 


\section{DETECTOR GROUP INFORMATION}

Detector Group Name
Detector Group Configuration
Analysis Date
Energy Calibration
Efficiency Calibration
Multi Curve
Source Distribution
Analysis Sequence File
Bkg Acq used for area correction
Nuclide Identification
Nuclide Identification Library
Tentative NID Library
Peak Search Library
Analysis Limits (channels)
Energy Tolerance (FWHM)
Comment

Summed

Thyroid Screening

3/23/2011 3:08:58 PM

Energy Calibration (I-125) 3/16/2011 3:02 PM

Efficiency Calibration (I-125) 3/16/2011 4:31 PM (Empirical)

No

Thyroid

Thyroid ASF for I-125.ASF

Not Performed

I-125 Thyroid.nlb

I-125 Thyroid.nlb

I-125 Thyroid.nlb

$20-4095$

1.20

PEAK SEARCH RESULTS FOR Accuscan II <Summed>

\begin{tabular}{|c|c|c|c|c|c|c|c|c|c|}
\hline \multicolumn{6}{|c|}{ Peak Analysis } & \multicolumn{4}{|c|}{ Nuclide Information } \\
\hline & No & \begin{tabular}{|l} 
Peak \\
Centroid
\end{tabular} & $\begin{array}{l}\text { Energy } \\
(\mathrm{keV})\end{array}$ & $\begin{array}{l}\text { Net Peak } \\
\text { Area }\end{array}$ & \begin{tabular}{|l} 
Continuum \\
Counts
\end{tabular} & \begin{tabular}{|l} 
Nuclide \\
Name
\end{tabular} & Activity (nCi) & $\begin{array}{l}\text { Error } \\
\text { (1SD) }\end{array}$ & $\begin{array}{l}\text { Yield } \\
(\%)\end{array}$ \\
\hline M & 1 & 59.77 & 27.28 & $3.36 \mathrm{E}+003$ & $9.29 \mathrm{E}+002$ & $\mathrm{I}-125$ & $9.859657 \mathrm{E}+001$ & $3.75 \%$ & 40.25 \\
\hline $\mathrm{m}$ & 2 & 67.89 & 31.04 & $8.51 \mathrm{E}+002$ & $5.16 \mathrm{E}+002$ & $\mathrm{I}-125$ & $1.026382 \mathrm{E}+002$ & $9.08 \%$ & 12.50 \\
\hline $\mathrm{m}$ & 3 & 77.23 & 35.36 & $2.48 \mathrm{E}+002$ & $2.24 \mathrm{E}+002$ & Unknown & $4.359149 \mathrm{E}+000$ & & 100.00 \\
\hline
\end{tabular}

For unknown peaks we assume yield to be $100 \%$ and no decay correction is performed.

$M=$ First peak in a multiplet region

$\mathrm{m}=$ Other peak in a multiplet region

$\mathrm{F}=$ Fitted singlet

NUCLIDE RESULTS FOR Accuscan II <Summed>

\begin{tabular}{|l|l|l|l|r|r|r|r|}
\hline & $\begin{array}{l}\text { Nuclide } \\
\text { Name }\end{array}$ & $\begin{array}{l}\text { Id } \\
\text { Confidence }\end{array}$ & $\begin{array}{l}\text { Wt Mean } \\
\text { Activity (nCi) }\end{array}$ & Error (1SD) & Action Level 1 & Action Level 2 & MDA (nCi) \\
\hline $\mathrm{I}-125$ & 0.997 & $9.914710 \mathrm{E}+001$ & $3.47 \%$ & Not Performed & Not Performed & $5.971 \mathrm{E}+000$ \\
\hline
\end{tabular}

$?=$ Nuclide is part of an undetermined solution

$\mathrm{X}=$ Nuclide rejected by the interference analysis

@ = Nuclide contains energy lines not used in Weighted Mean Activity

! = Nuclide was corrected for parent/daughter

\section{NUCLIDE MDA RESULTS FOR Accuscan II <Summed>}

\begin{tabular}{|c|c|c|c|c|c|c|}
\hline & Nuclide & Energy (keV) & Yield (\%) & Line MDA & Nuclide MDA & Activity (nCi) \\
\hline+ & $1-125$ & 27.00 * & 40.25 & $5.9705 E+000$ & $5.9705 E+000$ & $9.8597 \mathrm{E}+001$ \\
\hline+ & & 31.00 * & 12.50 & $1.8374 \mathrm{E}+001$ & & $1.0264 \mathrm{E}+002$ \\
\hline
\end{tabular}




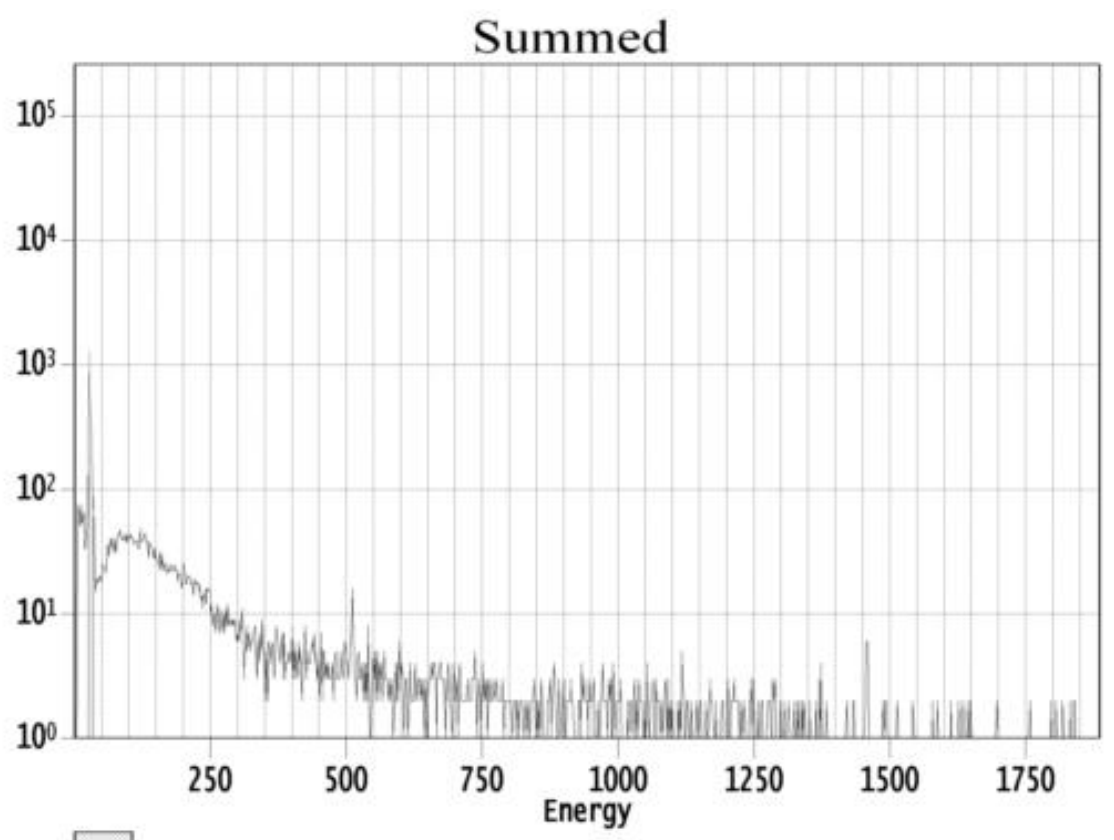

ROI Type: 2 


\section{COUNT 2}

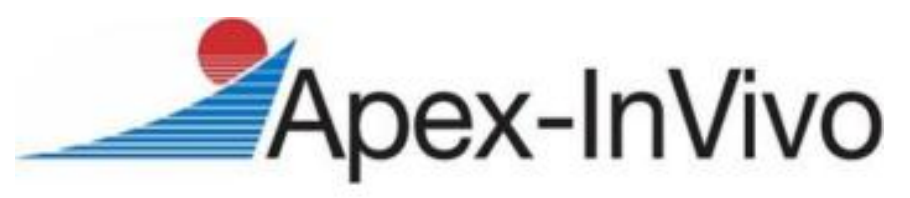

\section{Analysis Report - I-125 Validation Count [000-125]}

\section{SOURCE INFORMATION}

Primary ID

Last Name

000-125

First Name

Validation Count

Height (in)

I-125

Weight (lb)

Chest Wall Thickness (cm)

\section{COUNTER INFORMATION}

Counter Name

Counter Location

Configuration Name
Accuscan II

Battelle

Thyroid - I-125

\section{COUNT INFORMATION}

Count Operation

Count Reason

Frequency

Comment

Intake Date

Acquisition Started

Acquisition Time

Operator Name

Count Status

Primary Review

Secondary Review
Individual 10 minutes

Routine operation

3/23/2011 3:25:54 PM

601.42

David Georgeson

Not Reviewed

No

No

\section{DETECTOR COUNT RATE REPORT}

\begin{tabular}{|l|r|r|r|c|}
\hline \multicolumn{1}{|c|}{ Detector Name } & $\begin{array}{c}\text { Count } \\
\text { Rate }\end{array}$ & $\begin{array}{c}\text { Count } \\
\text { Rate }\end{array}$ & Live Time & Analyzed \\
\hline Right & 20.18 & $1-4096$ & 600.0 & No \\
\hline Left & 18.42 & $1-4096$ & 600.0 & No \\
\hline Summed & 38.58 & $1-4096$ & 600.0 & Yes \\
\hline
\end{tabular}

Primary Review by:

Date:

User: David Georgeson 


\section{DETECTOR GROUP INFORMATION}

$\begin{array}{ll}\text { Detector Group Name } & \text { Summed } \\ \text { Detector Group Configuration } & \text { Thyroid Screening } \\ \text { Analysis Date } & \text { 3/23/2011 3:25:12 PM } \\ \text { Energy Calibration } & \text { Energy Calibration (I-125) 3/16/2011 3:02 PM } \\ \text { Efficiency Calibration } & \text { Efficiency Calibration (I-125) 3/16/2011 4:31 PM (Empirical) } \\ \quad \text { Multi Curve } & \text { No } \\ \text { Source Distribution } & \text { Thyroid } \\ \text { Analysis Sequence File } & \text { Thyroid ASF for I-125.ASF } \\ \text { Bkg Acq used for area correction } & \text { Not Performed } \\ \text { Nuclide Identification } & \\ \quad \text { Nuclide Identification Library } & \text { I-125 Thyroid.nlb } \\ \quad \text { Tentative NID Library } & \text { I-125 Thyroid.nlb } \\ \quad \text { Peak Search Library } & \text { I-125 Thyroid.nlb } \\ \quad \text { Analysis Limits (channels) } & \text { Energy Tolerance (FWHM) } \\ \quad \text { Comment } & 1.20\end{array}$

\section{PEAK SEARCH RESULTS FOR Accuscan II <Summed>}

\begin{tabular}{|r|r|r|r|r|r||}
\hline \multicolumn{7}{|c|}{ Peak Analysis } \\
\hline & No & $\begin{array}{l}\text { Peak } \\
\text { Centroid }\end{array}$ & $\begin{array}{l}\text { Energy } \\
\text { (keV) }\end{array}$ & $\begin{array}{l}\text { Net Peak } \\
\text { Area }\end{array}$ & \multicolumn{2}{l|}{$\begin{array}{l}\text { Continuum } \\
\text { Counts }\end{array}$} \\
\hline & 1 & 38.01 & 17.22 & $1.76 \mathrm{E}+002$ & $2.72 \mathrm{E}+002$ \\
\hline $\mathrm{M}$ & 2 & 59.80 & 27.30 & $3.33 \mathrm{E}+003$ & $8.10 \mathrm{E}+002$ \\
\hline $\mathrm{m}$ & 3 & 67.75 & 30.98 & $8.74 \mathrm{E}+002$ & $4.47 \mathrm{E}+002$ \\
\hline $\mathrm{m}$ & 4 & 77.55 & 35.51 & $2.67 \mathrm{E}+002$ & $1.90 \mathrm{E}+002$ \\
\hline
\end{tabular}

For unknown peaks we assume yield to be $100 \%$ and no decay correction is performed.

$M=$ First peak in a multiplet region

$\mathrm{m}=$ Other peak in a multiplet region

$\mathrm{F}=$ Fitted singlet

NUCLIDE RESULTS FOR Accuscan II <Summed>

\begin{tabular}{|l|l|l|l|r|}
\hline $\begin{array}{l}\text { Nuclide } \\
\text { Name }\end{array}$ & $\begin{array}{l}\text { Id } \\
\text { Confidence }\end{array}$ & $\begin{array}{l}\text { Wt Mean } \\
\text { Activity (nCi) }\end{array}$ & Error (1SD) \\
\hline I-125 & 0.997 & $9.878515 \mathrm{E}+001$ & $3.47 \%$ \\
\hline
\end{tabular}

\section{NUCLIDE MDA RESULTS FOR Accuscan II <Summed>}

\begin{tabular}{|c|c|c|c|c|}
\hline & Nuclide & Energy (keV) & Yield (\%) & Line MDA \\
\hline+ & \multirow[t]{2}{*}{ |-125 } & 27.00 * & 40.25 & $5.5885 E+000$ \\
\hline+ & & 31.00 * & 12.50 & $1.7075 \mathrm{E}+001$ \\
\hline
\end{tabular}

$+=$ Nuclide Identified during the nuclide identification

* = Energy Line found in the spectrum 


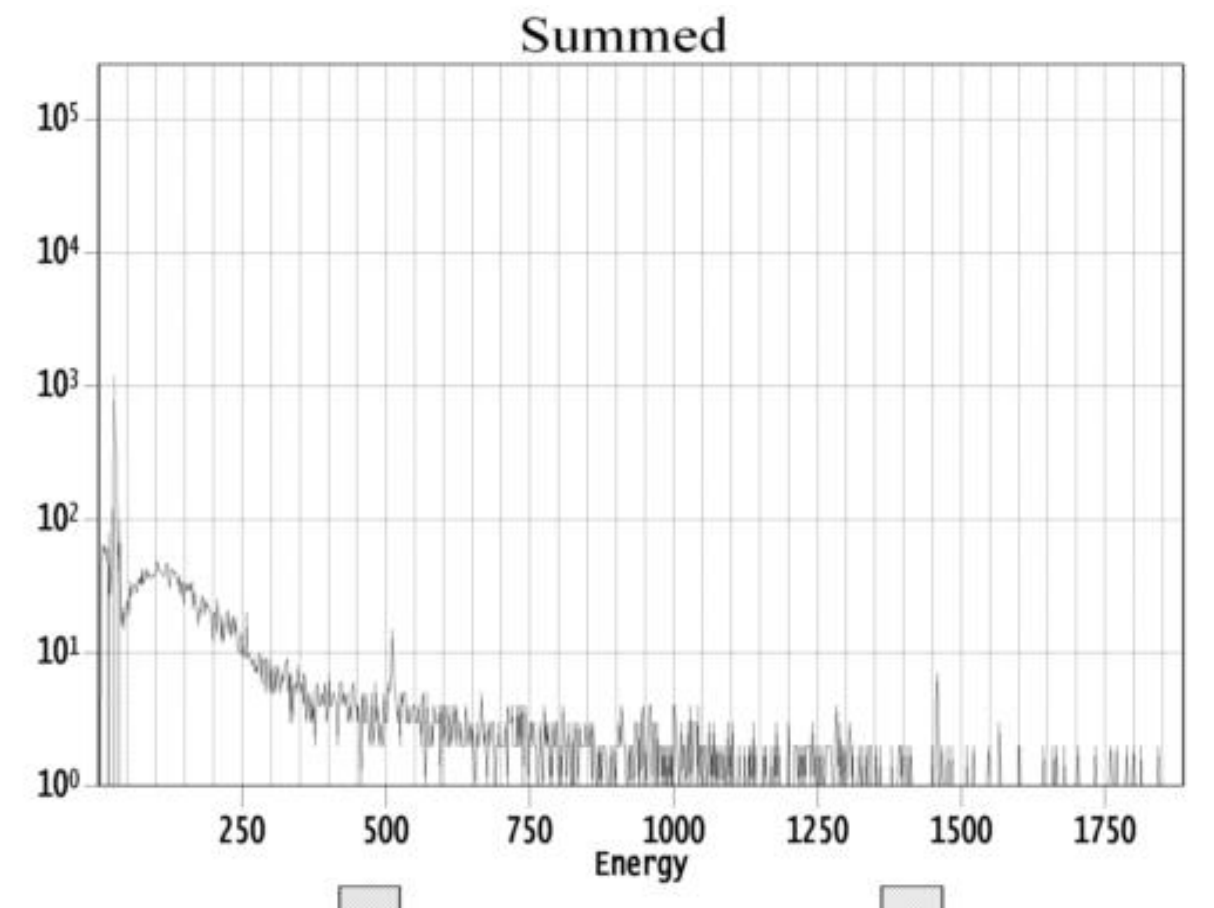

ROI Type: 1

ROI Type: 2

User: David Georgeson 


\section{COUNT 3}

\section{Apex-InVi}

\section{Analysis Report - I-125 Validation Count [000-125]}

\section{SOURCE INFORMATION}

Primary ID

Last Name

First Name

Height (in)

Weight (Ib)

Chest Wall Thickness $(\mathrm{cm})$
000-125

Validation Count

I-125

\section{COUNTER INFORMATION}

Counter Name

Counter Location

Configuration Name
Accuscan II

Battelle

Thyroid - I-125

\section{COUNT INFORMATION}

\section{Count Operation}

Count Reason

Frequency

Comment

Intake Date

Acquisition Started

Acquisition Time

Operator Name

Count Status

Primary Review

Secondary Review
Individual 10 minutes

Routine operation

$$
\begin{aligned}
& \text { 3/23/2011 3:41:45 PM } \\
& 601.39 \\
& \text { David Georgeson } \\
& \text { Not Reviewed } \\
& \text { No } \\
& \text { No }
\end{aligned}
$$

\section{DETECTOR COUNT RATE REPORT}

\begin{tabular}{|l|c|c|c|c|l|l|}
\hline \multicolumn{1}{|c|}{ Detector Name } & $\begin{array}{c}\text { Count } \\
\text { Rate }\end{array}$ & $\begin{array}{c}\text { Count } \\
\text { Rate }\end{array}$ & Live Time & $\begin{array}{c}\text { Analyze } \\
\text { d }\end{array}$ & Analyzed Date & File Name \\
\hline Left & 18.73 & $1-4096$ & 600.0 & No & & 88d02fc81541.cnf \\
\hline Right & 19.87 & $1-4096$ & 600.0 & No & & $348 \mathrm{~d} 689 \mathrm{~d} 1541 . \mathrm{cnf}$ \\
\hline Summed & 38.53 & $1-4096$ & 600.0 & Yes & $3 / 23 / 20113: 41: 36$ & $644 \mathrm{e} 5 \mathrm{f} 9 \mathrm{c} 1541 . \mathrm{cnf}$ \\
\hline
\end{tabular}

Primary Review by:

Date:
Secondary Review by:

Date: 


\section{DETECTOR GROUP INFORMATION}

Detector Group Name

Detector Group Configuration

Analysis Date

Energy Calibration

Efficiency Calibration

Multi Curve

Source Distribution

Analysis Sequence File

Bkg Acq used for area correction

Nuclide Identification

Nuclide Identification Library

Tentative NID Library

Peak Search Library

Analysis Limits (channels)

Energy Tolerance (FWHM)

Comment
Summed

Thyroid Screening

3/23/2011 3:41:36 PM

Energy Calibration (I-125) 3/16/2011 3:02 PM

Efficiency Calibration (I-125) 3/16/2011 4:31 PM (Empirical)

No

Thyroid

Thyroid ASF for I-125.ASF

Not Performed

I-125 Thyroid.nlb

I-125 Thyroid.nlb

I-125 Thyroid.nlb

$20-4095$

1.20

PEAK SEARCH RESULTS FOR Accuscan II <Summed>

\begin{tabular}{|c|c|c|c|c|c|c|c|c|c|}
\hline \multicolumn{6}{|c|}{ Peak Analysis } & \multicolumn{4}{|c|}{ Nuclide Information } \\
\hline & No & \begin{tabular}{|l} 
Peak \\
Centroid
\end{tabular} & $\begin{array}{l}\text { Energy } \\
(\mathrm{keV})\end{array}$ & $\begin{array}{l}\text { Net Peak } \\
\text { Area }\end{array}$ & $\begin{array}{l}\text { Continuum } \\
\text { Counts }\end{array}$ & $\begin{array}{l}\text { Nuclide } \\
\text { Name }\end{array}$ & Activity (nCi) & Error (1SD) & $\begin{array}{l}\text { Yield } \\
(\%)\end{array}$ \\
\hline M & 1 & 59.79 & 27.29 & $3.26 \mathrm{E}+003$ & $8.19 E+002$ & I-125 & $9.564766 \mathrm{E}+0$ & $3.76 \%$ & 40.25 \\
\hline $\mathrm{m}$ & 2 & 67.83 & 31.01 & $8.46 \mathrm{E}+002$ & $6.11 \mathrm{E}+002$ & I-125 & $1.018599 \mathrm{E}+0$ & $9.10 \%$ & 12.50 \\
\hline $\mathrm{m}$ & 3 & 77.51 & 35.49 & $2.19 \mathrm{E}+002$ & $2.87 E+002$ & Unknown & $3.853039 \mathrm{E}+0$ & & 100.00 \\
\hline
\end{tabular}

For unknown peaks we assume yield to be $100 \%$ and no decay correction is performed.

$M=$ First peak in a multiplet region

$\mathrm{m}=$ Other peak in a multiplet region

$\mathrm{F}=$ Fitted singlet

NUCLIDE RESULTS FOR Accuscan II <Summed>

\begin{tabular}{|l|l|l|l|r|l|l|l|}
\hline & $\begin{array}{l}\text { Nuclide } \\
\text { Name }\end{array}$ & $\begin{array}{l}\text { Id } \\
\text { Confidence }\end{array}$ & $\begin{array}{l}\text { Wt Mean } \\
\text { Activity (nCi) }\end{array}$ & Error (1SD) & Action Level 1 & Action Level 2 & MDA (nCi) \\
\hline $\mathrm{I}-125$ & 0.997 & $9.645958 \mathrm{E}+001$ & $3.47 \%$ & Not Performed & Not Performed & $5.614 \mathrm{E}+000$ \\
\hline
\end{tabular}

$?=$ Nuclide is part of an undetermined solution

$\mathrm{X}=$ Nuclide rejected by the interference analysis

@ = Nuclide contains energy lines not used in Weighted Mean Activity

! = Nuclide was corrected for parent/daughter

\section{NUCLIDE MDA RESULTS FOR Accuscan II <Summed>}

\begin{tabular}{|c|c|c|c|c|c|c|}
\hline & $\begin{array}{l}\text { Nuclide } \\
\text { Name }\end{array}$ & Energy (keV) & Yield (\%) & $\begin{array}{l}\text { Line MDA } \\
(\mathrm{nCi})\end{array}$ & $\begin{array}{l}\text { Nuclide MDA } \\
\text { (nCi) }\end{array}$ & Activity (nCi) \\
\hline+ & \multirow[t]{2}{*}{$\mid-125$} & $27.00 *$ & 40.25 & $5.6137 \mathrm{E}+000$ & \multirow[t]{2}{*}{$5.6137 \mathrm{E}+000$} & $9.5648 \mathrm{E}+001$ \\
\hline+ & & $31.00 *$ & 12.50 & $1.9942 \mathrm{E}+001$ & & $1.0186 \mathrm{E}+002$ \\
\hline
\end{tabular}

$+=$ Nuclide Identified during the nuclide identification

* = Energy Line found in the spectrum 


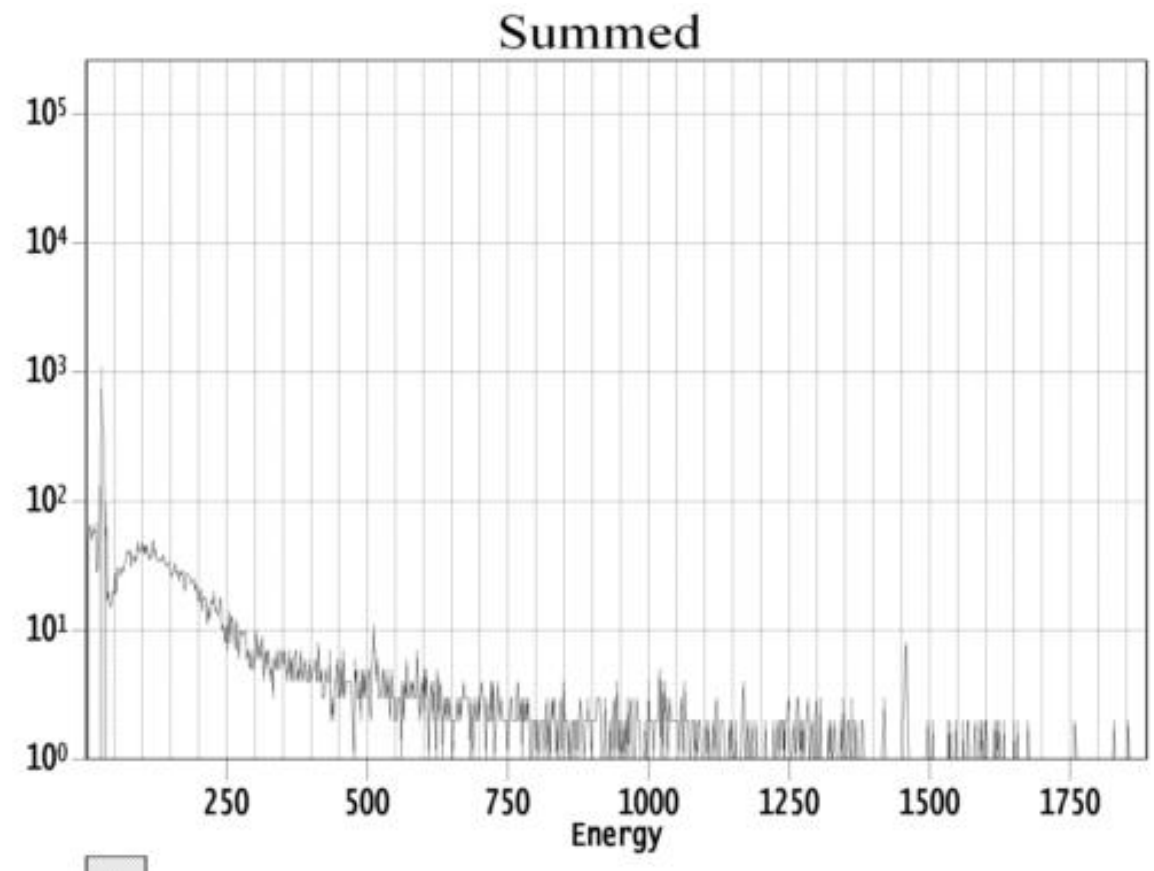

ROI Type: 2 


\section{Analysis Report - I-125 Validation Count [000-125]}

\section{SOURCE INFORMATION}

Primary ID

Last Name

First Name

Height (in)

Weight (lb)

Chest Wall Thickness (cm)
000-125

Validation Count

I-125

\section{COUNTER INFORMATION}

\section{Counter Name \\ Counter Location \\ Configuration Name}

Accuscan II

Battelle

Thyroid - I-125

\section{COUNT INFORMATION}

\begin{tabular}{ll}
$\begin{array}{l}\text { Count Operation } \\
\text { Count Reason } \\
\text { Frequency } \\
\text { Comment } \\
\text { Intake Date }\end{array}$ & $\begin{array}{l}\text { Individual } 10 \text { minutes } \\
\text { Routine operation }\end{array}$ \\
Acquisition Started & \\
Acquisition Time & \\
Operator Name & $3 / 23 / 20113: 53: 09$ PM \\
Count Status & 601.41 \\
Primary Review & David Georgeson \\
Secondary Review & Not Reviewed \\
& No \\
\hline
\end{tabular}

DETECTOR COUNT RATE REPORT

\begin{tabular}{|l|c|c|c|c|l|l|}
\hline Detector Name & $\begin{array}{c}\text { Count } \\
\text { Rate }\end{array}$ & $\begin{array}{c}\text { Count } \\
\text { Rate }\end{array}$ & Live Time & Analyzed & Analyzed Date & File Name \\
\hline Left & 18.83 & $1-4096$ & 600.0 & No & & $5 a 8847391553 . c n f$ \\
\hline Right & 19.97 & $1-4096$ & 600.0 & No & & $6 f 5 c 18931553 . c n f$ \\
\hline Summed & 38.48 & $1-4096$ & 600.0 & Yes & $3 / 23 / 20113: 53: 00$ & b314935e1553.cnf \\
\hline
\end{tabular}

Primary Review by:

Date:
Secondary Review by:

Date: 


\section{DETECTOR GROUP INFORMATION}

$\begin{array}{ll}\text { Detector Group Name } & \text { Summed } \\ \text { Detector Group Configuration } & \text { Thyroid Screening } \\ \text { Analysis Date } & 3 / 23 / 2011 \text { 3:53:00 PM } \\ \text { Energy Calibration } & \text { Energy Calibration (I-125) 3/16/2011 3:02 PM } \\ \text { Efficiency Calibration } & \text { Efficiency Calibration (I-125) 3/16/2011 4:31 PM (Empirical) } \\ \quad \text { Multi Curve } & \text { No } \\ \text { Source Distribution } & \text { Thyroid } \\ \text { Analysis Sequence File } & \text { Thyroid ASF for I-125.ASF } \\ \text { Bkg Acq used for area correction } & \text { Not Performed } \\ \text { Nuclide Identification } & \\ \quad \text { Nuclide Identification Library } & \text { I-125 Thyroid.nlb } \\ \quad \text { Tentative NID Library } & \text { I-125 Thyroid.nlb } \\ \quad \text { Peak Search Library } & \text { I-125 Thyroid.nlb } \\ \quad \text { Analysis Limits (channels) } & 20-4095 \\ \quad \text { Energy Tolerance (FWHM) } & 1.20 \\ \text { Comment } & \end{array}$

PEAK SEARCH RESULTS FOR Accuscan II <Summed>

\begin{tabular}{|c|c|c|c|c|c|c|c|c|c|}
\hline \multicolumn{6}{|c|}{ Peak Analysis } & \multicolumn{4}{|c|}{ Nuclide Information } \\
\hline & No & \begin{tabular}{|l} 
Peak \\
Centroid
\end{tabular} & \begin{tabular}{|l|} 
Energy \\
(keV)
\end{tabular} & \begin{tabular}{|l} 
Net Peak \\
Area
\end{tabular} & \begin{tabular}{|l|} 
Continuum \\
Counts
\end{tabular} & $\begin{array}{l}\text { Nuclide } \\
\text { Name }\end{array}$ & Activity (nCi) & Error (1SD) & $\begin{array}{l}\text { Yield } \\
(\%)\end{array}$ \\
\hline & 1 & 38.53 & 17.46 & $8.02 \mathrm{E}+001$ & $2.98 \mathrm{E}+002$ & Unknown & $1.426229 \mathrm{E}-$ & & 100.00 \\
\hline M & 2 & 59.82 & 27.31 & $3.31 \mathrm{E}+003$ & $7.88 \mathrm{E}+002$ & $\mathrm{I}-125$ & $9.731969 \mathrm{E}+0$ & $3.74 \%$ & 40.25 \\
\hline $\mathrm{m}$ & 3 & 67.75 & 30.97 & $8.15 E+002$ & $5.12 \mathrm{E}+002$ & $\mathrm{I}-125$ & $9.802611 \mathrm{E}+0$ & $9.08 \%$ & 12.50 \\
\hline $\mathrm{m}$ & 4 & 77.34 & 35.41 & $2.74 \mathrm{E}+002$ & $1.99 \mathrm{E}+002$ & Unknown & $4.817942 \mathrm{E}+0$ & & 100.00 \\
\hline
\end{tabular}

For unknown peaks we assume yield to be $100 \%$ and no decay correction is performed.

$M=$ First peak in a multiplet region

$\mathrm{m}=$ Other peak in a multiplet region

$\mathrm{F}=$ Fitted singlet

NUCLIDE RESULTS FOR Accuscan II <Summed>

\begin{tabular}{|c|c|c|c|c|c|c|}
\hline \begin{tabular}{|l|}
$\begin{array}{l}\text { Nuclide } \\
\text { Name }\end{array}$ \\
\end{tabular} & \begin{tabular}{|l} 
Id \\
Confidence
\end{tabular} & $\begin{array}{l}\text { Wt Mean } \\
\text { Activity (nCi) }\end{array}$ & Error (1SD) & Action Level 1 & Action Level 2 & MDA (nCi) \\
\hline $\mathrm{I}-125$ & 0.997 & $9.742112 \mathrm{E}+00$ & $3.46 \%$ & Not Performed & Not Performed & $5.516 \mathrm{E}+000$ \\
\hline
\end{tabular}

\section{NUCLIDE MDA RESULTS FOR Accuscan II <Summed>}

\begin{tabular}{|c|c|c|c|c|c|c|}
\hline & Nuclide & Energy (keV) & Yield (\%) & Line MDA & Nuclide MDA & Activity (nCi) \\
\hline+ & \multirow[t]{2}{*}{$\mid-125$} & 27.00 * & 40.25 & $5.5162 \mathrm{E}+000$ & \multirow[t]{2}{*}{$5.5162 E+000$} & $9.7320 \mathrm{E}+001$ \\
\hline+ & & 31.00 * & 12.50 & 1.8259E+001 & & $9.8026 \mathrm{E}+001$ \\
\hline
\end{tabular}




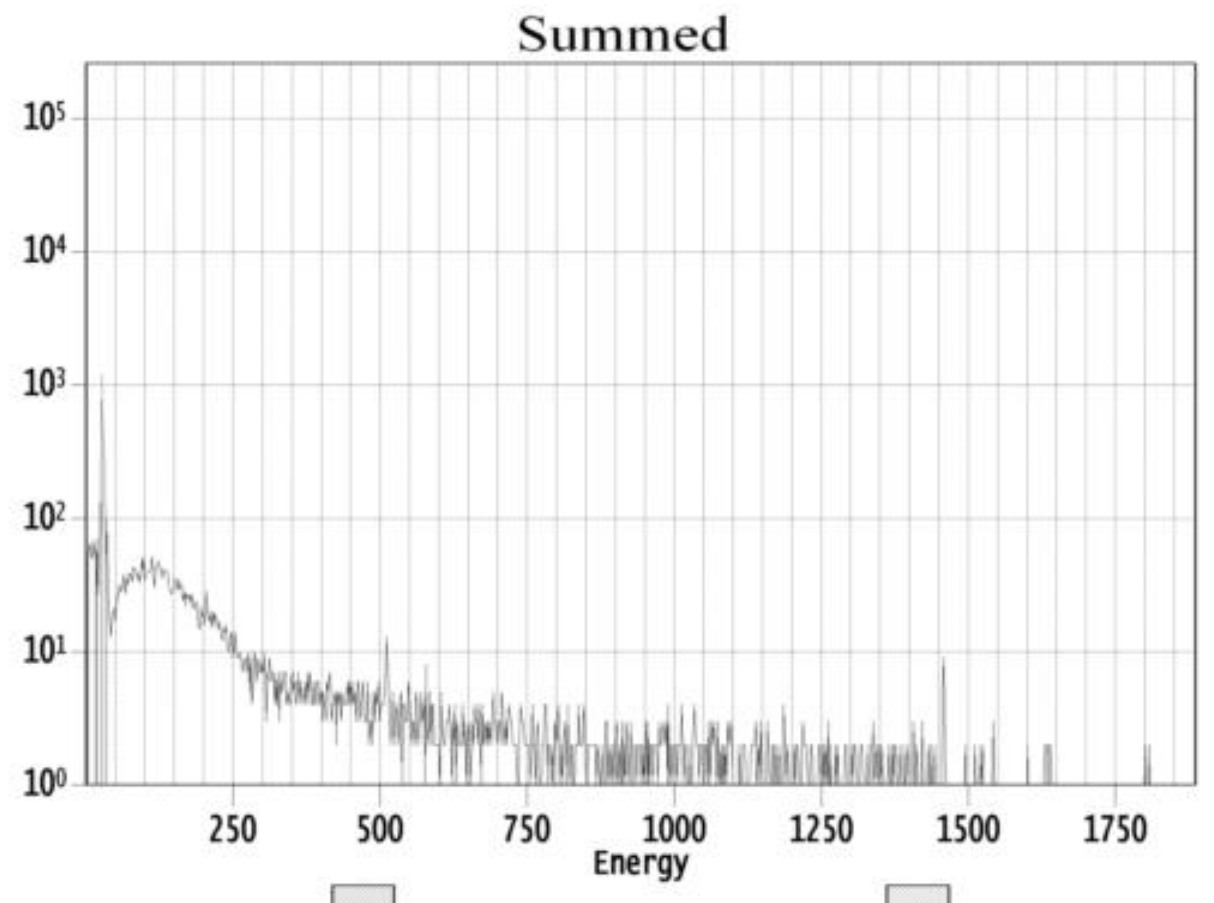

ROI Type: 1

ROI Type: 2 


\section{COUNT 5}

Apex-InVivo

\section{Analysis Report - I-125 Validation Count [000-125]}

\section{SOURCE INFORMATION}

Primary ID

Last Name

First Name

Height (in)

Weight (Ib)

Chest Wall Thickness (cm)
000-125

Validation Count

I-125

\section{COUNTER INFORMATION}

Counter Name

Counter Location

Configuration Name

\section{Accuscan II}

Battelle

Thyroid - I-125

\section{COUNT INFORMATION}

\section{Count Operation \\ Count Reason \\ Frequency \\ Comment \\ Intake Date \\ Acquisition Started \\ Acquisition Time \\ Operator Name \\ Count Status \\ Primary Review \\ Secondary Review}

Individual 10 minutes

Routine operation

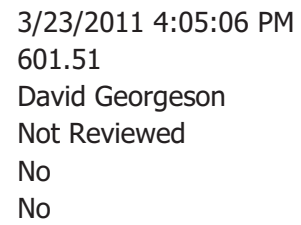

No

DETECTOR COUNT RATE REPORT

\begin{tabular}{|l|r|r|r|c|}
\hline \multicolumn{1}{|c|}{ Detector Name } & \multicolumn{1}{c|}{ Count Rate } & $\begin{array}{c}\text { Count Rate } \\
\text { Range }\end{array}$ & Live Time & Analyzed \\
\hline Left & 18.59 & $1-4096$ & 600.0 & No \\
\hline Right & 19.96 & $1-4096$ & 600.0 & No \\
\hline Summed & 38.39 & $1-4096$ & 600.0 & Yes \\
\hline
\end{tabular}

Primary Review by:

Date:

User: David Georgeson 


\section{DETECTOR GROUP INFORMATION}

\begin{tabular}{ll} 
Detector Group Name & Summed \\
Detector Group Configuration & Thyroid Screening \\
Analysis Date & $3 / 23 / 2011$ 4:04:58 PM \\
Energy Calibration & Energy Calibration (I-125) 3/16/2011 3:02 PM \\
Efficiency Calibration & Efficiency Calibration (I-125) 3/16/2011 4:31 PM (Empirical) \\
$\quad$ Multi Curve & No \\
Source Distribution & Thyroid \\
Analysis Sequence File & Thyroid ASF for I-125.ASF \\
Bkg Acq used for area correction & Not Performed \\
Nuclide Identification & \\
$\quad$ Nuclide Identification Library & I-125 Thyroid.nlb \\
$\quad$ Tentative NID Library & I-125 Thyroid.nlb \\
$\quad \begin{array}{l}\text { Peak Search Library } \\
\text { Analysis Limits (channels) } \\
\text { Energy Tolerance (FWHM) }\end{array}$ & I-125 Thyroid.nlb \\
Comment & $20-4095$ \\
\hline
\end{tabular}

PEAK SEARCH RESULTS FOR Accuscan II <Summed>

\begin{tabular}{|r|r|r|r|r|r||}
\hline \multicolumn{7}{|c||}{ Peak Analysis } \\
\hline & \multicolumn{1}{|c|}{ No } & Peak Centroid & Energy (keV) & Net Peak Area & \multicolumn{2}{l||}{ Continuum Counts } \\
\hline $\mathrm{M}$ & 1 & 59.81 & 27.30 & $3.29 \mathrm{E}+003$ & $7.58 \mathrm{E}+002$ \\
\hline $\mathrm{m}$ & 2 & 67.85 & 31.02 & $7.15 \mathrm{E}+002$ & $5.62 \mathrm{E}+002$ \\
\hline$m$ & 3 & 77.46 & 35.47 & $2.34 \mathrm{E}+002$ & $2.71 \mathrm{E}+002$ \\
\hline & 4 & 3165.65 & 1459.62 & $3.79 \mathrm{E}+001$ & $1.61 \mathrm{E}+001$ \\
\hline
\end{tabular}

For unknown peaks we assume yield to be $100 \%$ and no decay correction is performed.
$\mathrm{M}=$ First peak in a multiplet region
$\mathrm{m}=$ Other peak in a multiplet region
$\mathrm{F}=$ Fitted singlet

\section{NUCLIDE RESULTS FOR Accuscan II <Summed>}

\begin{tabular}{rl|l|l|l|}
\hline $\begin{array}{l}\text { Nuclide } \\
\text { Name }\end{array}$ & $\begin{array}{l}\text { Id } \\
\text { Confidence }\end{array}$ & Wt Mean Activity (nCi) & Error (1SD) \\
\hline I-125 & 0.997 & $9.487243 \mathrm{E}+001$ & $3.47 \%$ \\
\hline
\end{tabular}

\section{NUCLIDE MDA RESULTS FOR Accuscan II <Summed>}

\begin{tabular}{|c|c|c|c|c|}
\hline & Nuclide & Energy (keV) & Yield (\%) & Line MDA (nCi) \\
\hline+ & $\mid-125$ & 27.00 * & 40.25 & $5.4099 \mathrm{E}+000$ \\
\hline+ & & $31.00 *$ & 12.50 & $1.9153 \mathrm{E}+001$ \\
\hline
\end{tabular}

$+=$ Nuclide Identified during the nuclide identification

* = Energy Line found in the spectrum 


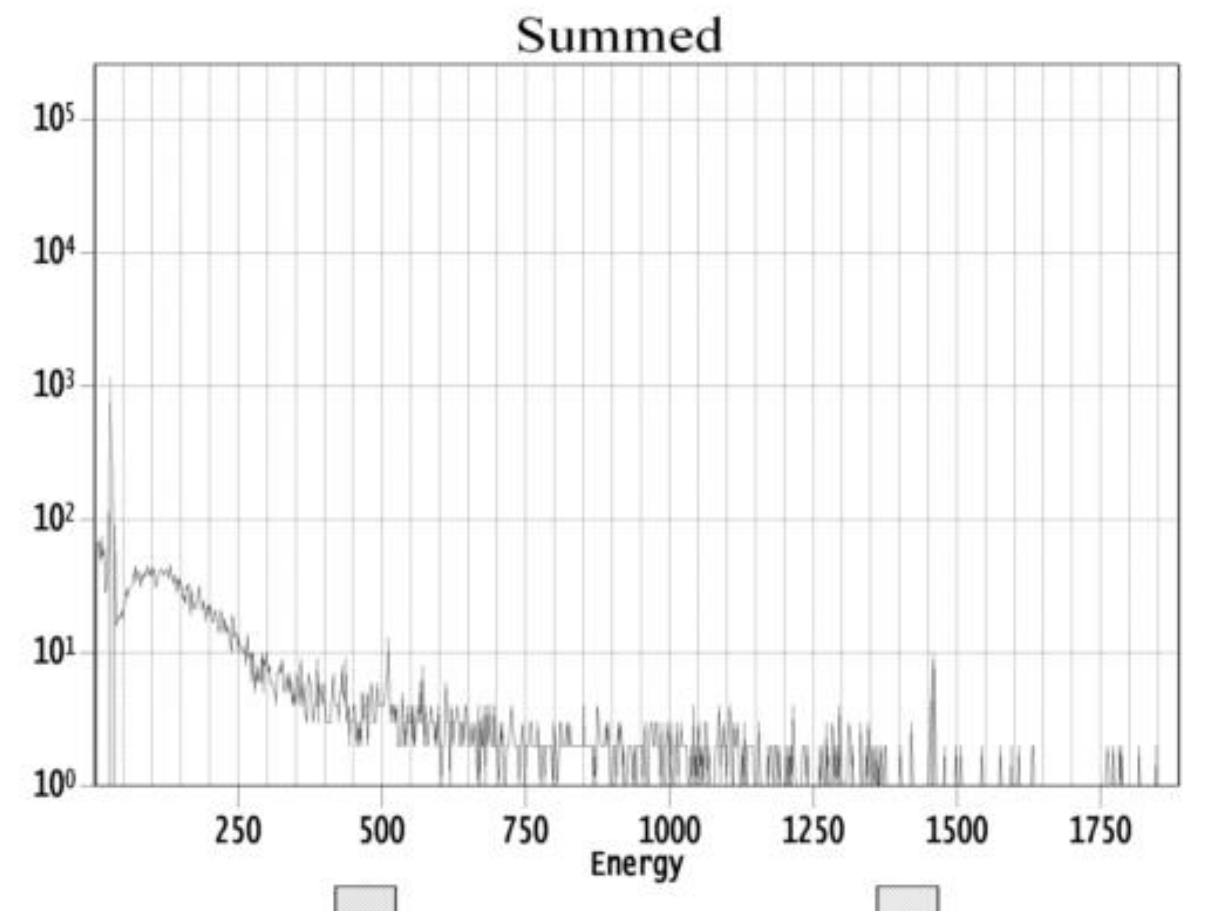

ROI Type: 1

ROI Type: 2 


\section{Appendix I}

ANSI N44.3 Phantom 


\section{Appendix I ANSI N44.3 Phantom}

Abetrast

\section{American National Standard Thyrold Radiolodine Uptake Measurements Using a Neck Phantom \\ Seretetarios}

thureav of Radislogical Mroth of the U.S. Publie Hanth Sovice

Aoprosd Angur 24, 1ara

Ameriean Notienal Standards Instituta, Inc.

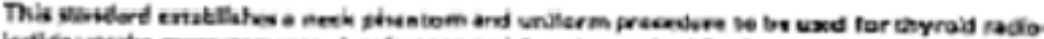

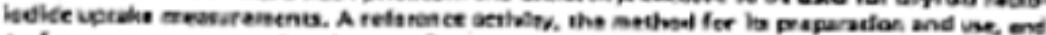

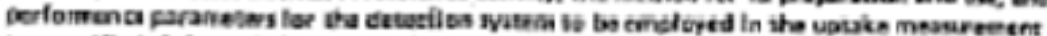

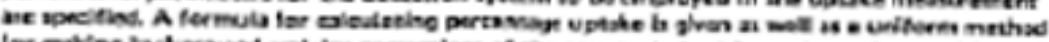

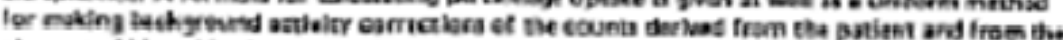

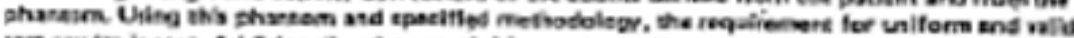

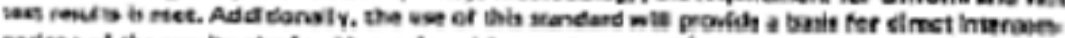

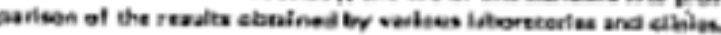

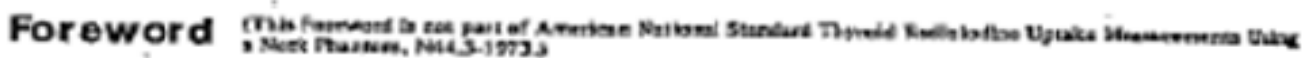

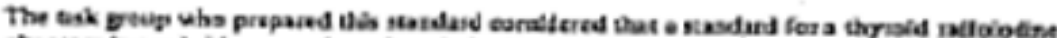

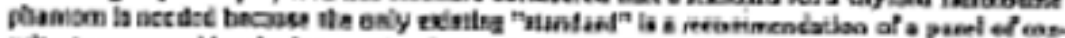

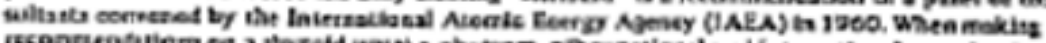

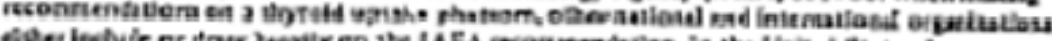

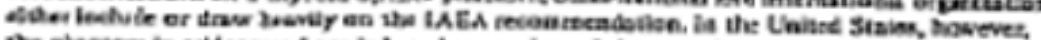

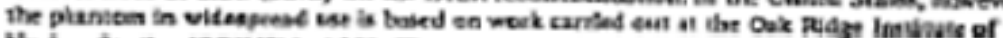

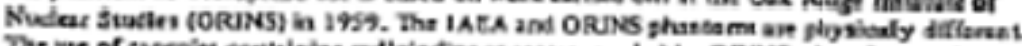

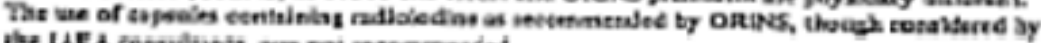
Lie LER cekouliahis, wa noi seconmesided.

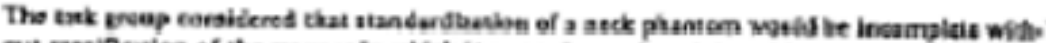

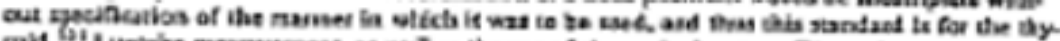

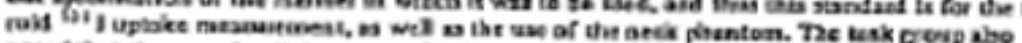

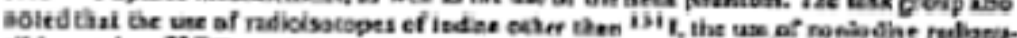

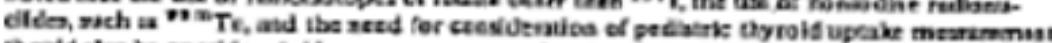

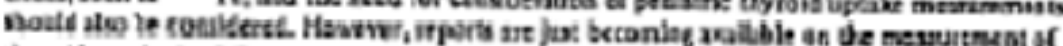

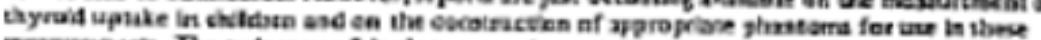

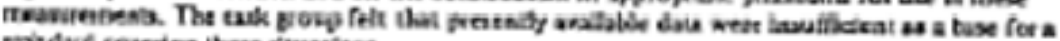
saidare ceverieg thas stuations.

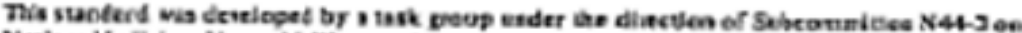

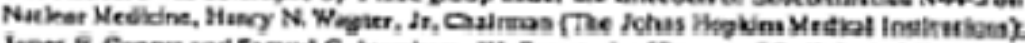

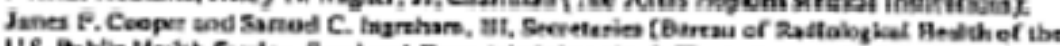

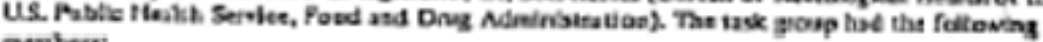
aumbers:

Froer nomarwedaks, Crarran

(Pverto Res Nadrar Cenier)

Ficterd C Keny, Socretury

(Ulelveratiy of Kenses Muaca!

Cancer) it Glane:

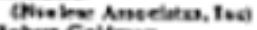

acber Cellewe.

Cibrentig of Teses

C. Cals thanis

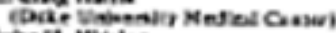

leno. Undy

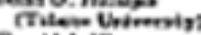

Goress 1. thes

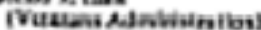

Ualle 1. Mathicis:

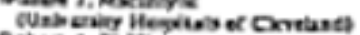
Ausea A. Pib

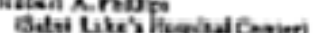
Truesere Vinfole

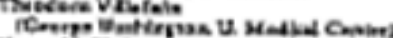

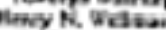

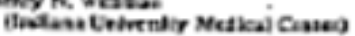




\section{American National Standard Thyroid $R$ adioiodine Uptake Measurements Using a Neck Phantom}

\begin{abstract}
I. Scope
This standard entabilabes the refrence activiny (usura)

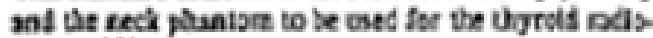

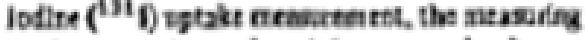
cquphest to be used, ane nose procedural appects of the sese of the neck phasason in te mtareremests that ace segulied to obtain vild lest rmalts. No ellairal

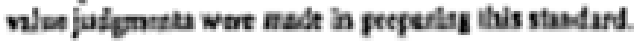

\section{Definftions}

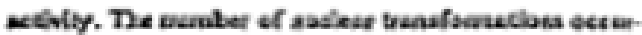
rins per unit time.

"B" titer. A lrad stceld das b gbeed apinst the neck

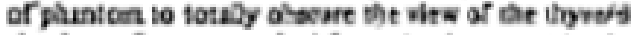
vasd er infereece anodand frem to deiester. The dt. meadous cf be "B" ftier as $10.2 \times 10.2 \times 13 \mathrm{cs}$ $(4 \times 4 \times 1 / 2 \mathrm{la})$.

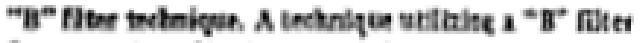
for correctica of backsound and exinaty reifl act. vity.

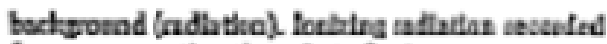
froen socices other the that of prirnary concers.

neferace astivity. De astivity esed in the setk plan.

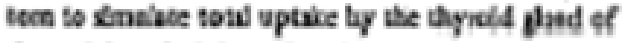
De ataivity afininisiered so the potlest.

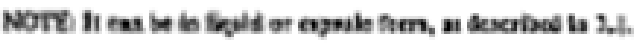
Ayroid ugtake. The tyrodt oplake (T), apiessed a o persenbie; thet is

$\mathrm{TU}=\frac{P-P_{3}}{S-S_{a}} \times 100 \times \mathrm{K}$

sherv

$P=$ couts per wait time fron the potien.

$P_{y}=$ eousts per unt tors from the posiest xith tha "irnitite is place

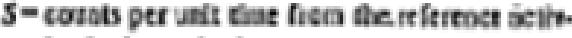
ity in the atck phastem

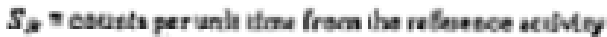
is tha arck ghenton with be " $B$ " SIIter is pias $K$ a ratio of referetce acilvery to the tothoy ad. mialtered ts the pulient
\end{abstract}

\section{Reference Aetivity and Nick Phanton}

\subsection{Referense Activing}

3.1.1 Tie pelerence setivity thal how the sane sctivity of ratoledie as a atminlseits is be potirzt

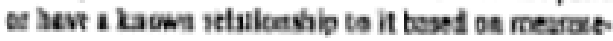
ernis.

3.1 .2 y a llyaid itfeitnse uctiky bs wed, ita rolamt that be $30 \mathrm{~m}$.

3.13 ar a apule referrose octivity is tard, If etall be eilher: (1) dhooluted in waier to make a liguld refer-

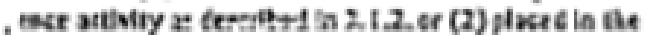
apeih solder (uet 3.2.4) fer Irstuba into the atk phattem.

3.1.4 Tevesul for the lieteid reiernin attivity

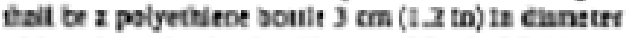

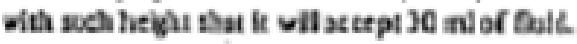

\subsection{Nuck Imaton}

32.1 Te nodk pianien, lliatrated in Fig. 1, shal be a righ cqlinder $12.7 \mathrm{~cm}$ (5 b) in dlameter and 12.7 an (5 in) in height tnd athall be male of polpmrityl

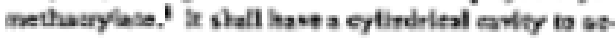
sept the refestoce activity cescoited ia 3.1 .

3.2.2 Te natial diatsnes fram the surfoce of the pheaien to the mefuce of the cavily ilal be $0.5 \mathrm{~cm}$. 0.2 in $)$ The tentrd wis of be cavity ahall be $2.0 \mathrm{~cm}$ (Q.8 in) frse the arlase of the phteism (see Fit 1).

3.2 .3 Teet ftal te a flat sarfate on the phatiom

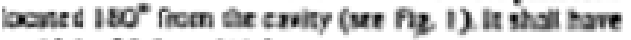
1 videt of $2.3 \mathrm{cn}(1 \mathrm{tn})$.

2.24 A reass dabl be providel to boll the ernier of the capeale referesca artility at ihe seometulad cenier of the liqutd tefereate attivity aite within 0.

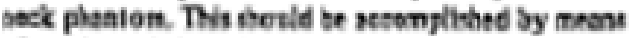
of a jolyme ityl methacrylace lolict of the dineaslots

\footnotetext{
Tor aunds, bedie is Rer tha.
} 

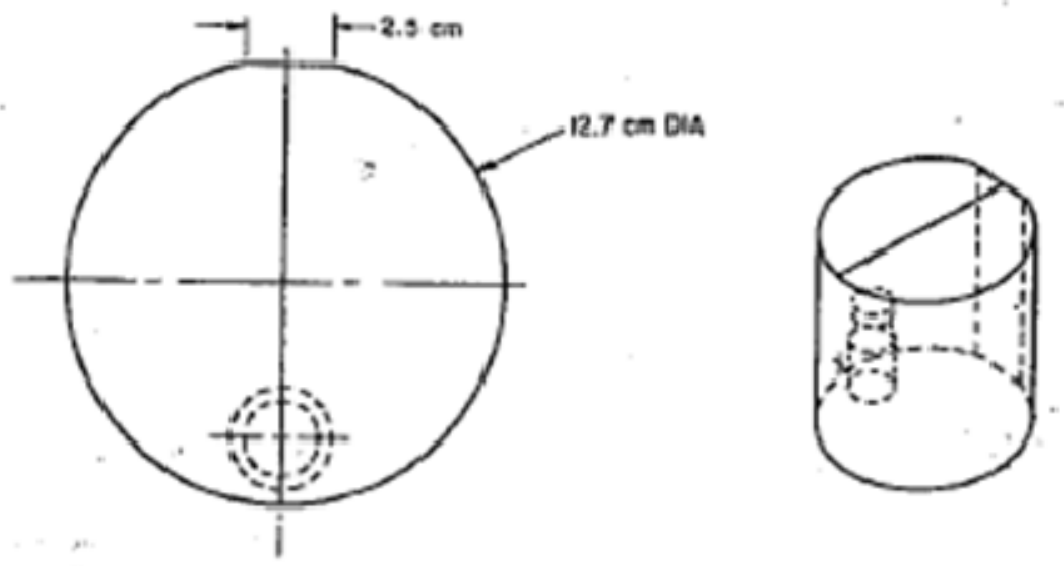

MATERIAL: POLYMETHFL NETHACRYLTE

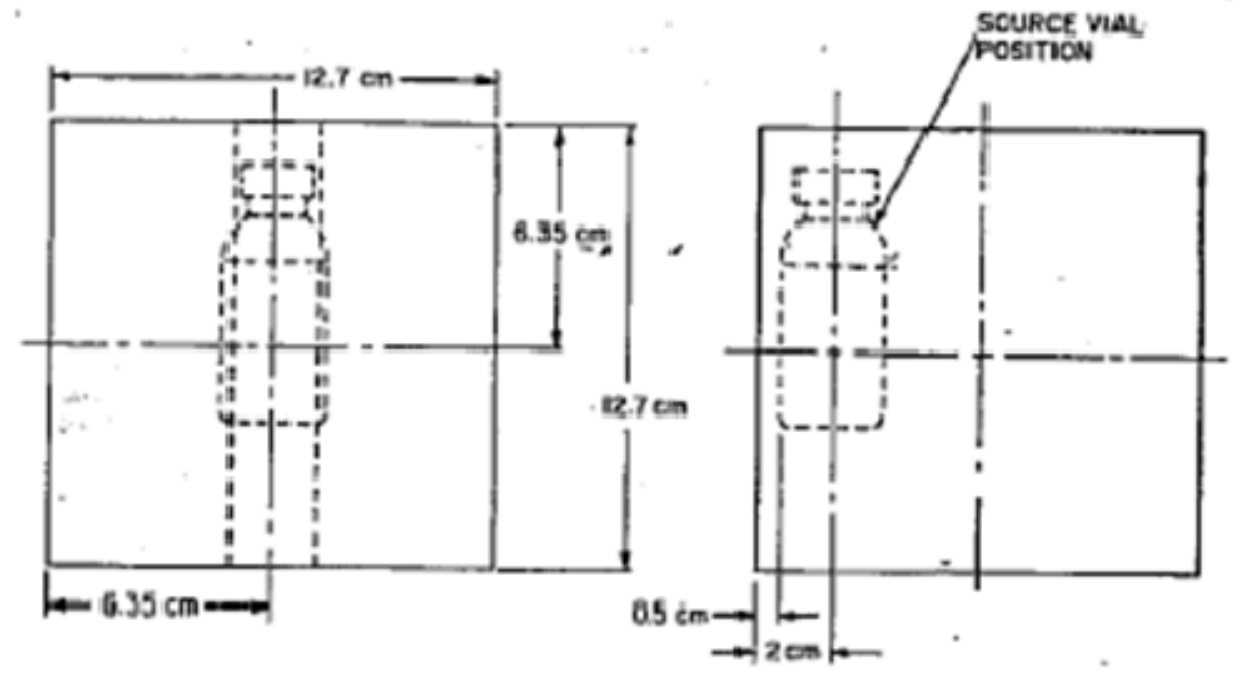

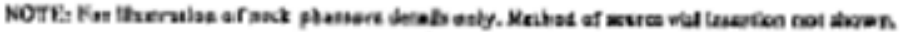

Fin. 1

Nock Fasilom fer Thyold tpiak:

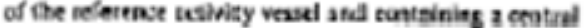
tele of eporcpaiat dtannter sad stepth.

3.23 The phentom shall contala narked cenirning Ines wi its suiface that carsspots to the cronterled

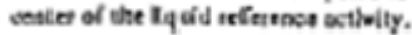

3.26 Tht phenion and any arsessy aw llary parts shall be des'catd io permit tha ligald refirtasn

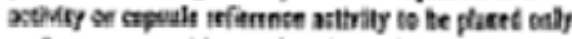
at the poser position withle the seck pleaton. 3.2 .7 A ncaus ball be previted lo minintir air 
ppe within tit seck phanien when eiber the liquid itfertice sttivity or tapule referenere activing is is alase.

\section{Eqaipment}

4.1 Detneter. The datetier stould te a $\mathrm{Nat}$ (TI) erys tal sot less tas $2.5 \mathrm{en}$ ( $\mathrm{t}$ in) in any dimeasien.

\section{4,2 Calinatse}

4.2.1 The collinator should bave a fielt of vitw at die selested pattent-destecter distarioe (tre 5.1.2) that will siequately eacempas the reglos of ate dlyroid thend, but aot intredsa bry regions of eatrethyrolat sctikty. $A$ Celd ef virw with adius $R$ at leas $6 \mathrm{~cm}$ and

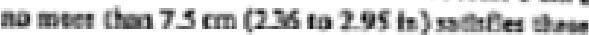
modintoes te mat cass. Mupite bis toeld of vikw, the

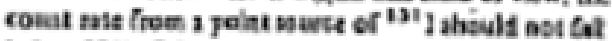
belew $50 \pi$ of the mavinun toupt ratt at tee cenier of the fivis.

4.2 .2 The couat min insuld fall to $50 x$ or lows as the diatarce foom the als lecreases to $1.2 R$ and to 52 or irin as thts dtstatce itrseasts to $1, A R$. For this ins suruest tha soure thul teltally le kealed on the

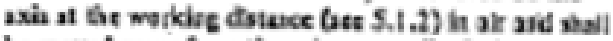

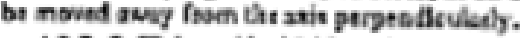

4.23 Sumititst side wiel ding should he provilad so that the couse nie eal fal yelew $1 \%$ af the ines.

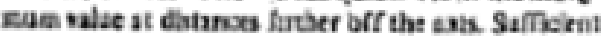

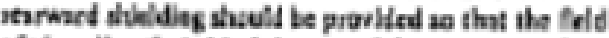
af Viru directly behlad tit cryatal don not excesd ir

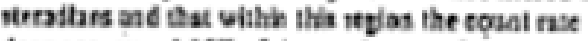
doer sot excetd ISA of tin maxirosan vatue.

\subsection{Cewatleg Eevipenax}

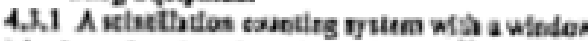
(shiptehumel analyzer) or a lower level discrimtator showis be used.

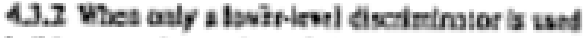
is abal be act no lewer der $250 \mathrm{xeV}$.

\section{Procedure}

\subsection{Datung tetweea racent ind Detector}

5.1.1 The Clstatce tesween the potinnt and the

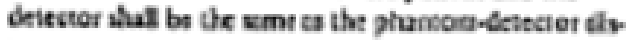
tater.
5.1.2 The Cintante that be neasurt frem tat mo face of the fritecior to tha thin overiping the ieferiat

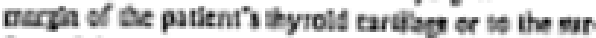
fuce of the natk phorsen. The daturce shuid se te twons 25 and $50 \mathrm{sm}(10$ io $20 \mathrm{in})$.

\subsection{Thot of Mosaicsincent}

S.2.1 A 24hoar apteke mesurentrt can yield sel.

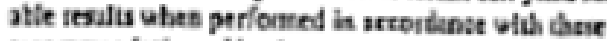
ieconetendations. Upcake mascarratents made carlie: thon $\$$ beva after aflriabtration of ralieiedine mag

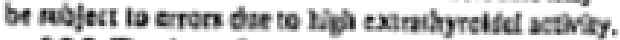

5.2.2 The tirce of aneauetrnens atouls be ledudrt is a staturunt of thyodid aptake; for esurigle, 24tr TU(S), 6te TU (5), th:

\subsection{Letisity Rykgone}

5.3.1 Vile radloctivity in the glent is teirg ceuted, batkgoand wasts tae so rotm baxkgroind. ptotass frús the potient that pans troust the deistior Alallits and ochir extachyroidal actolty within the field of wrw ofl be rtenidnd. These my te aurnod together by whe the "E" Btei technique and Aw pes vite a strde odntection for roon bockyeted and all oater exurathyreits activing.

5.3 .2 The " 6 " Nutr hall be s piece of lead 10.3 $X 10.2 \times 1.2 \mathrm{~cm}$ thick $14 \times+\times 1 / 2$ tel.

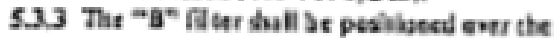

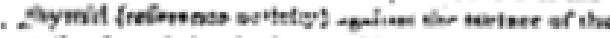

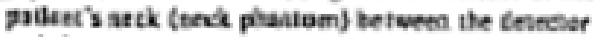
und the patient (resk phrsion).

5.4 Egutitu for Caleulados of Pertast Thyrold Up. the. Fercant ibyioit upate (TU) bial be caloubies fres the fellowing tqualion:

$\mathrm{TU}=\frac{r=r g}{s-3,} \times 100 \times K$

where

$P=$ cornts ger ualt tare foon the patien:

$P_{a}=$ couns atr usik tere fies the palltal wilk ibe "g" fliter is plast

$S$ - tounis per vea time from the velerene actinity It the nedk phenian

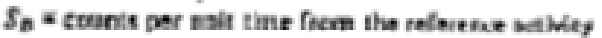
to the steck phanion with the " $\mathrm{B}$ " Gilier in piase

$Z$ * ntlo of reftiente activily to the activing atmin. ietered la the paitent

\section{NUCLEAD ASSOCIATES}

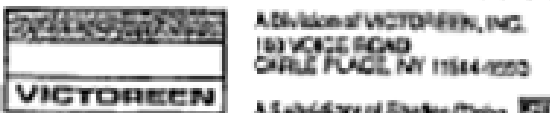

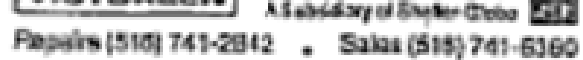



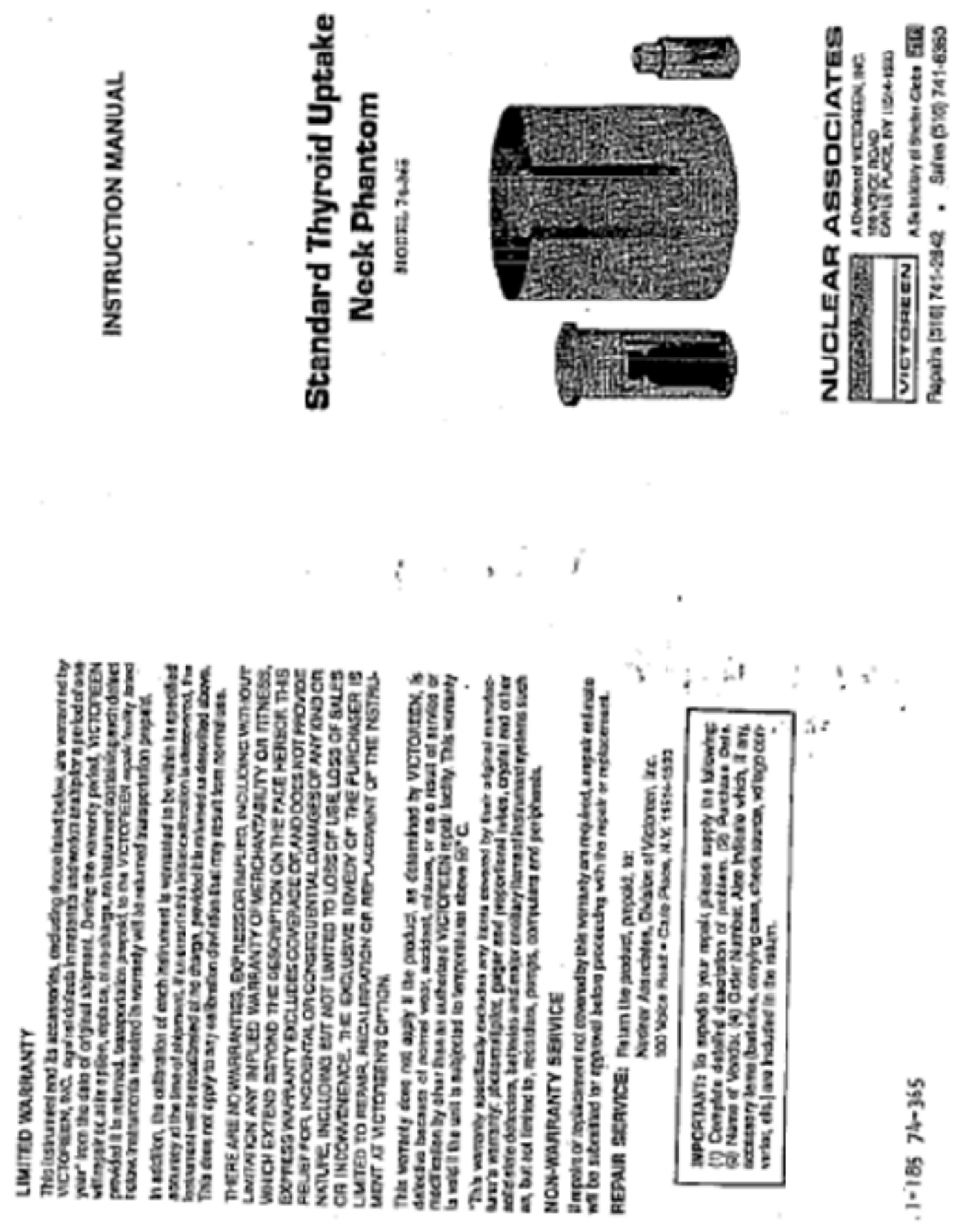

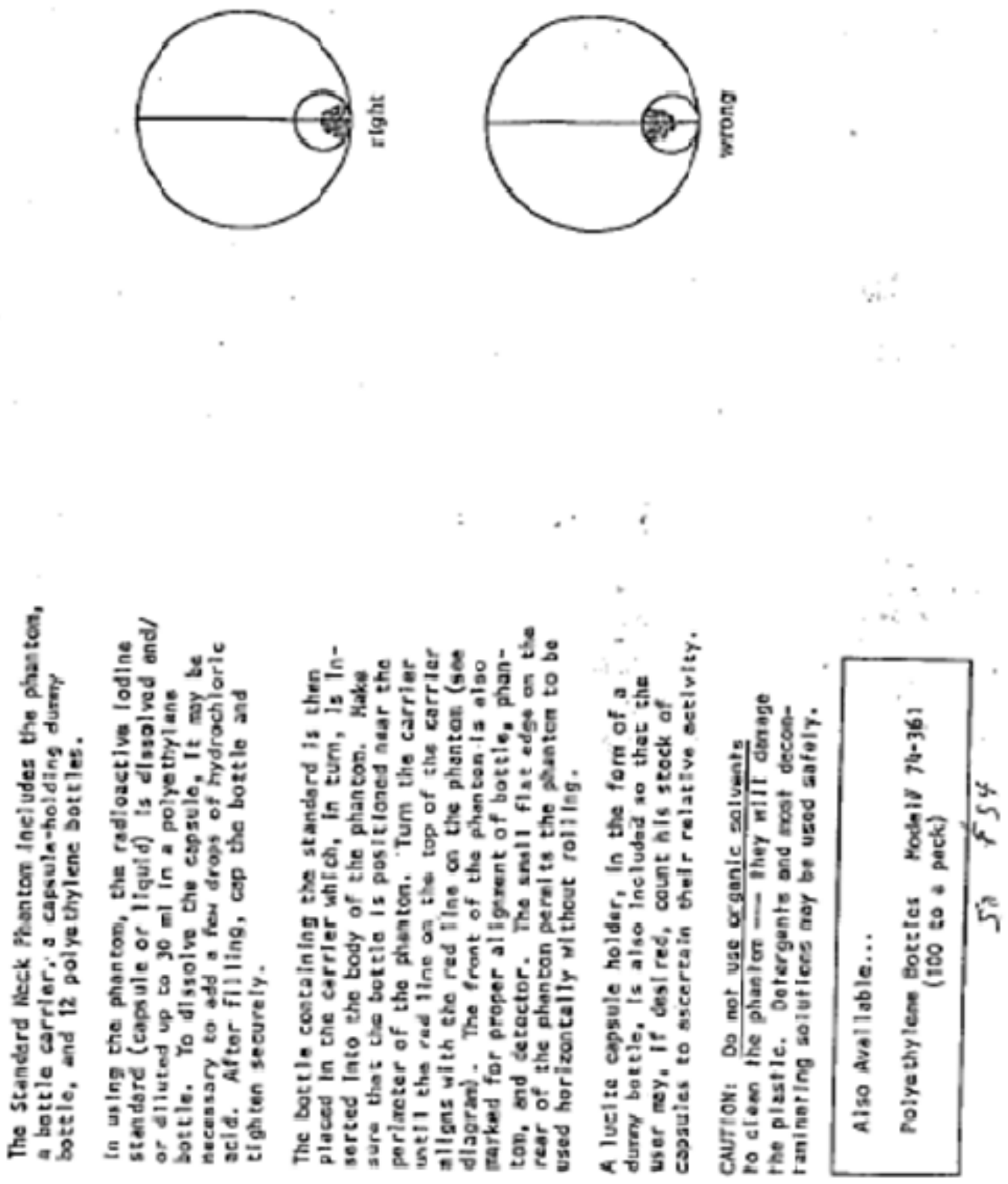
Appendix $\mathbf{J}$

RMC II Transfer Phantom 


\section{Appendix $\mathrm{J}$ RMC II Transfer Phantom}

The Canberra RMC-II

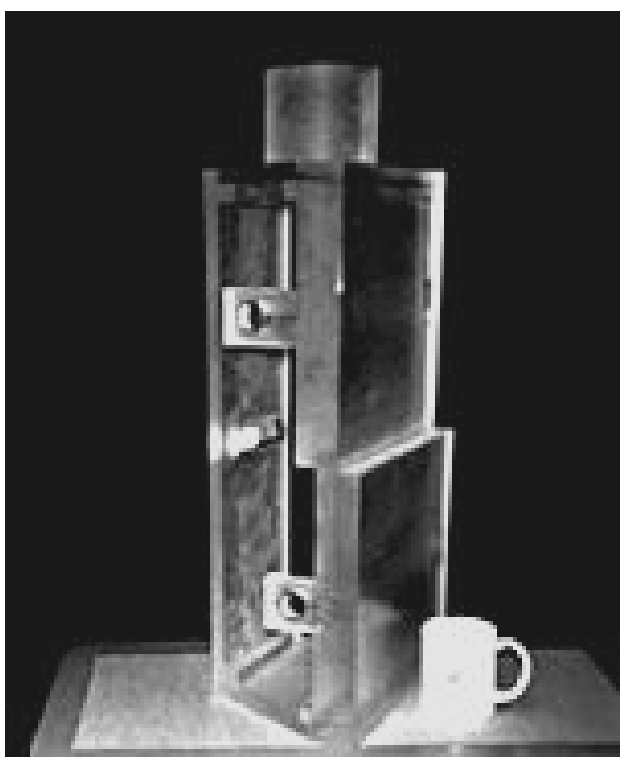

(Model 2257) Transfer Phantom

\section{J-1. CANBERRA RMC-II (MODEL 2257)TRANSFER PHANTOM}

This is an inexpensive and easy-to-use phantom that is intended for use during efficiency calibrations of Canberra's various "linear geometry" in vivo counters. This phantom has been designed to adequately duplicate the counting geometries of the ANSI N13.30 (later published as HPS N13.30-1996) reference phantom configurations when used to calibrate the standard Canberra Fastscan, Accuscan (Bed), and Accuscan-II counting systems. The reference phantom counting geometries duplicated by the Canberra Transfer Phantom include the Livermore Realistic torso lungs, the BOMAB total body, and the ANSI-N44.3 thyroid. The original test counts performed to verify the suitability of the Canberra Transfer Phantom are summarized in the following pages.

The Canberra Transfer Phantom can be used to accurately simulate lung, GI region, whole body, or thyroid source activity distributions. It is easy to use because it needs no assembly and requires only a single "mixed-gamma" source in a 20-ml liquid scintillation vial for all calibration geometries.

This phantom can provide accurate efficiency calibration results when properly used with standard Canberra Fastscan, Accuscan (Bed), and Accuscan-II counting systems, as well with standard Nuclear Data "People Mover" systems. This phantom is not appropriate for calibrations involving low-energy photon measurements (attempted quantification of nuclide activity based on detection of photons with energies less than $100 \mathrm{keV}$ ), or for counting geometries involving one or more detectors positioned behind the subject.

\section{J-2. VERIFICATION OF THE Canberra RMC-II CALIBRATION Phantom}

For proper calibration of whole body counter (WBC) systems, it is necessary to simulate the photon emanation and absorption characteristics of a human counting subject with internally deposited radioactive material. This is accomplished by loading standard nuclides of known activities in a 
"phantom" absorber matrix, placed at a designated position relative to the shield and detectors of the WBC system.

Canberra has developed an inexpensive and easy-to-handle phantom appropriate for calibrating linear-geometry fission and activation product WBC systems. This phantom closely approximates the source/absorber configurations provided by the more expensive and complex reference phantoms specified by ANSI N13.30 (later published as HPS N13.30-1996) and ANSI N44.3 as appropriate for WBC calibrations. This phantom is intended for use with small-volume radioactive sources (typically prepared in one-inch diameter liquid scintillation vials). These sources may be loaded in any one of four standard positions within the phantom, thereby simulating reference configurations for lung, thyroid, G.I. region, and whole body activity distributions. This phantom is hereafter referred to as the "RMC-II" phantom [with the "II" added to indicate a significant design change from the REMCAL Transfer Phantom previously developed by Radiation Management Corporation (RMC) prior to acquisition of RMC by Canberra].

The RMC-II phantom consists of two basic components: a "torso" section, and a "neck" section. The torso section is constructed from flat sheets of cast acrylic material (e.g., Lexan or Plexiglass), with the front sheet thickness chosen to provide the proper amount of absorber between the calibration source and the WBC system detector(s). Three interior source cavities provide lung-equivalent, G.I. region-equivalent, and whole body-equivalent configurations. The neck section is a cast acrylic cylinder with a thyroid-equivalent source cavity, with dimensions specified in ANSI N44.3. This configuration provides the ANSI-approved reference thyroid counting geometry.

The RMC-II phantom must be properly positioned in the WBC shield when performing calibration counts. The base of the torso section must be placed near "waist level" of a reference counting subject in the WBC shield. For Canberra Fastscan and Accuscan-II counters, the base of the torso section must be flat against the rear interior shield wall, centered between the molded guide ridges, at a height of 36 inches above the interior shield floor pad. For Canberra Accuscan (Bed) systems, the back of the phantom must be centered and flat on the bed pad, and the base of the torso section placed 36 inches from the foot plate.

\section{J-3. PRELIMINARY TESTING OF THE RMC-II PHANTOM}

The optimum values of cast acrylic sheet thickness and source cavity dimensions were determined by direct comparison of WBC system response using ANSI-specified reference phantoms and an adjustable RMC-II phantom prototype. To determine the efficiency vs. photon energy response for the reference lung configuration, a Livermore Realistic torso phantom was used (purchased by RMC from Humanoid Systems). Lung inserts simulating a uniform activity distribution of Eu-152 were loaded in the phantom, and three chest wall thickness values ranging from $16 \mathrm{~mm}$ to $39 \mathrm{~mm}$ were used.

Initially, test counts were performed using a standard Fastscan system assembled with two $4 " \times 4 " \times 16$ " NaI detectors). Efficiency values were calculated for six prominent Eu-152 photopeak energies, and smooth-curve functions were fitted to these measured efficiency values. The efficiency curve for the $22.5 \mathrm{~mm}$ chest thickness configuration was accepted as the reference "lung efficiency" response to be duplicated by the RMC-II phantom lung configuration.

Next, a series of test counts was performed using a Eu-152 point source and various thickness values of cast acrylic absorber. The source was mounted at a position near the midpoint of the lungs in a reference subject, 54 inches above the interior shield floor pad. The thickness of absorber material between the source and the detectors was varied in 0.25 -inch increments until the resulting efficiency values fit a smooth curve with shape similar to that of the reference lung efficiency curve. The cast acrylic sheets were sufficiently large to intercept all straight-line photon paths between the source and detectors. The distance between the source and the back wall of the Fastscan shield was then varied until the efficiency values calculated for the RMC-II phantom were in good agreement with those for the reference 
phantom. This process resulted in the selection of 1.85 inches of acrylic absorber and 3.5 inches distance from back of the phantom to source midline as the optimum dimensions for simulating the Livermore Realistic phantom lung configuration (with $22.5 \mathrm{~mm}$ chest wall thickness).

A second series of test counts was performed to simulate the reference "BOMAB" whole body phantom configuration. For this activity distribution, a 10-piece cylindrical and ellipsoidal polyethylene jug phantom was filled with uniform activity concentration Eu-152 solution (60 liters total volume). This 10-component phantom represents the head, neck, upper torso, arms, lower torso, thighs and lower legs of a counting subject. The phantom was supported in a standing configuration in the Fastscan shield, and efficiency values were determined for six photon energies as done previously for the reference lung configuration. Test counts performed with the adjustable RMC-II phantom prototype resulted in the selection of 1.85 inches of acrylic absorber, and 0.5 inches distance from the back of the phantom to the source midline, as the optimum dimensions for simulating the reference BOMAB whole body configuration.

A third series of test counts was performed to determine an appropriate G.I. region configuration for the RMC-II phantom. Because of the dynamic nature of the G.I. tract, and the lack of a specific ANSI recommendation for simulating this organ, defining a "reference" G.I. region phantom is somewhat problematic. Test counts were performed using the following three phantoms: (1) the Livermore Realistic torso phantom with 19 line-geometry sources loaded in the abdominal insert, (2) the Alderson Research Laboratories REMCAL phantom with a single line-geometry source loaded in the "G.I. cavity", and (3) the 10-piece BOMAB polyethylene jug body phantom with Eu-152 activity distributed in the lower torso component only. Efficiency values obtained with these three phantoms were fitted with smooth-curve functions.

Test counts were then performed with the adjustable RMC-II phantom prototype. The absorber thickness and source position was varied until the resulting efficiency values closely approximated the values obtained with the lower torso component of the 10-piece BOMAB phantom. (This also provided good agreement with the average of the Livermore Realistic abdominal cavity and REMCAL G.I. cavity efficiency values.) An absorber thickness of 1.85 inches and a 5.5-inch distance between the point source and back of the phantom were selected for the optimum RMC-II phantom G.I. region configuration, based on these comparative counts.

A production model of the RMC-II phantom was then prepared by Webb Plastics in Northbrook, Illinois. This production model of the RMC-II phantom was then tested using three standard Canberra linear-geometry WBC systems (Fastscan, Accuscan[Bed], and AccuScan-II) to confirm adequate similarity of the RMC-II phantom and the three reference phantoms, as explained below.

\section{J-4. VERIFICATION OF RMC-II PHANTOM DESIGN}

Final verification of the RMC-II phantom design was completed in February 1988. Comparative test counts were performed using three WBC systems at the Canberra Industries, Meriden, CT facility. The intent was to document RMC-II phantom and reference phantom equivalence for Fastscan systems, Accuscan Bed systems (when configured for horizontal scanning of the entire bed length) and AccuscanII systems (when configured for vertical scanning of the entire shield height).

The Livermore Realistic torso phantom, 10-piece BOMAB total body phantom, and lower torso component of the BOMAB phantom were again used as reference lung, whole body, and G.I. region phantom configurations, respectively. Eu-152 sources with activity distributions appropriate for each phantom were used in the comparative test counts. Efficiency values determined for the three counter types, using the reference phantoms and the RMC-II phantom production model, are summarized in Table J-1. Efficiency ratio values were calculated (RMC-II phantom efficiency divided by the corresponding reference phantom efficiency) and are also shown in Table J-1. 


\section{J-5. CONCLUSIONS}

Results of the verification test counts show acceptable agreement between the RMC-II phantom efficiency and the reference phantom efficiency for the three counter types tested. All efficiency ratio values listed in Table J-1 are within a range of 0.79-1.20, and the average ratio (all values weighted equally) is 1.02 .

When evaluating the performance of WBC systems, measured nuclide activity values will be inversely proportional to system efficiency values. If the RMC-II phantom is used for WBC system calibration, a bias in measured activity values will be expected, relative to those activity values that would have been calculated if the reference phantoms had been used for efficiency calibration. Table J-2 summarizes these relative bias values for each configuration and counter type tested.

The results shown in Table J-2 confirm that the RMC-II phantom provides an acceptable approximation of the ANSI-specified reference phantoms. All relative bias values shown are well within the WBC performance criteria limits for measurement accuracy specified in ANSI N13.30 (i.e., $-0.25<$ relative bias $<0.50$ ). Proper use of the RMC-II phantom will allow valid WBC system efficiency calibrations and ensure accurate WBC measurement results. A diagram showing the relative location of the four source cavities in the RMC-II phantom is shown in Figure J-1.

Table J-1. Summary of phantom comparison test count results.

\begin{tabular}{|c|c|c|c|c|c|c|c|}
\hline Geometry & Measured Response & $122 \mathrm{keV}$ & $245 \mathrm{keV}$ & $344 \mathrm{keV}$ & $779 \mathrm{keV}$ & $964 \mathrm{keV}$ & $1408 \mathrm{keV}$ \\
\hline \multirow[t]{3}{*}{ Lung $^{1}$} & Ref. Eff. ${ }^{4}(\% 2$ s.d.) & $8.21 \mathrm{E}-3(5.1)$ & 7.67E-3 (8.0) & $8.12 \mathrm{E}-3(2.4)$ & $5.95 \mathrm{E}-3(5.0)$ & 6.71E-3 (3.9) & $6.53 \mathrm{E}-3(1.9)$ \\
\hline & Test Eff. ( $\% 2$ s.d.) & $7.55 \mathrm{E}-3(5.0)$ & 7.98E-3 (8.4) & $7.52 \mathrm{E}-3(2.1)$ & 5.93E-3 (4.9) & $6.58 \mathrm{E}-3(3.2)$ & $6.32 \mathrm{E}-3(1.5)$ \\
\hline & Ratio +/- \% 2 s.d. & $0.92+/-7.1 \%$ & $1.04+/-12 \%$ & $0.93+/-3.2 \%$ & $1.00+/-7.1 \%$ & $0.98+/-5.0 \%$ & $0.97+/-2.4 \%$ \\
\hline \multirow[t]{3}{*}{ Total Body $^{2}$} & Ref. Eff. (\% 2 s.d.) & $5.16 \mathrm{E}-3(4.4)$ & $5.23 \mathrm{E}-3(8.9)$ & $5.57 \mathrm{E}-3(1.7)$ & $4.37 \mathrm{E}-3(4.6)$ & $4.81 \mathrm{E}-3(3.4)$ & $4.47 \mathrm{E}-3(1.5)$ \\
\hline & Test Eff. (\% 2 s.d.) & $5.56 \mathrm{E}-3(4.7)$ & $6.02 \mathrm{E}-3(5.3)$ & $5.73 \mathrm{E}-3(2.6)$ & $4.82 \mathrm{E}-3(5.0)$ & 5.37E-3 (2.6) & 4.83E-3 (1.2) \\
\hline & Ratio +/- \% 2 s.d. & $1.08+/-6.4 \%$ & $1.15+/-10 \%$ & $1.03+/-3.1 \%$ & $1.10+/-6.8 \%$ & $1.12+/-4.3 \%$ & $1.08+/-1.9 \%$ \\
\hline \multirow[t]{3}{*}{$\mathrm{GI}^{3}$} & Ref. Eff. (\% 2 s.d.) & 7.30E-3 (5.9) & $7.53 \mathrm{E}-3(9.6)$ & $8.11 \mathrm{E}-3(1.5)$ & $6.25 \mathrm{E}-3(5.4)$ & $6.77 \mathrm{E}-3(3.8)$ & $6.33 \mathrm{E}-3(1.4)$ \\
\hline & Test Eff. (\% 2 s.d.) & $7.43 \mathrm{E}-3(5.6)$ & 7.71E-3 (6.0) & $8.04 \mathrm{E}-3(2.5)$ & $6.83 \mathrm{E}-3(5.0)$ & $7.85 \mathrm{E}-3(2.8)$ & $7.15 \mathrm{E}-3(1.1)$ \\
\hline & Ratio +/- \% 2 s.d. & $1.02+/-8.1 \%$ & $1.02+/-11 \%$ & $0.99+/-2.9 \%$ & $1.09+/-7.4 \%$ & $1.16+/-4.7 \%$ & $1.13+/-1.8 \%$ \\
\hline \multirow[t]{3}{*}{ Lung } & Ref. Eff. (\% 2 s.d.) & 1.71 E-3 (9.2) & $2.04 \mathrm{E}-3(11.1)$ & $1.98 \mathrm{E}-3(3.4)$ & $1.38 \mathrm{E}-3(7.1)$ & $1.34 \mathrm{E}-3(7.6)$ & $1.44 \mathrm{E}-3(3.2)$ \\
\hline & Test Eff. (\% 2 s.d.) & 1.41 E-3 (10.3) & $1.95 \mathrm{E}-3$ (11.6) & $1.73 \mathrm{E}-3(3.9)$ & $1.34 \mathrm{E}-3(6.5)$ & $1.31 \mathrm{E}-3(5.7)$ & $1.37 \mathrm{E}-3(3.5)$ \\
\hline & Ratio $+/-\% 2$ s.d. & $0.82+/-14 \%$ & $0.96+/-16 \%$ & $0.87+/-5.2 \%$ & $0.97+/-9.6 \%$ & $0.98+/-9.5 \%$ & $0.95+/-4.7 \%$ \\
\hline \multirow[t]{3}{*}{ Total Body } & Ref. Eff. (\% 2 s.d.) & $1.51 \mathrm{E}-3(6.4)$ & $1.73 \mathrm{E}-3(7.7)$ & $1.69 \mathrm{E}-3(2.9)$ & $1.25 \mathrm{E}-3(6.0)$ & $1.27 \mathrm{E}-3(3.8)$ & $1.24 \mathrm{E}-3(2.0)$ \\
\hline & Test Eff. (\% 2 s.d.) & $1.46 \mathrm{E}-3(8.6)$ & $2.02 \mathrm{E}-3(6.3)$ & $1.70 \mathrm{E}-3(3.2)$ & $1.31 \mathrm{E}-3(5.6)$ & $1.30 \mathrm{E}-3(4.6)$ & $1.24 \mathrm{E}-3(2.4)$ \\
\hline & Ratio +/- \% 2 s.d. & $0.97+/-11 \%$ & $1.17+/-9.9 \%$ & $1.01+/-4.3 \%$ & $1.05+/-8.2 \%$ & $1.02+/-6.0 \%$ & $1.00+/-3.1 \%$ \\
\hline \multirow[t]{3}{*}{ GI } & Ref. Eff. (\% 2 s.d.) & $1.46 \mathrm{E}-3(8.3)$ & $1.69 \mathrm{E}-3(9.8)$ & 1.69E-3 (2.8) & $1.30 \mathrm{E}-3(4.8)$ & $1.28 \mathrm{E}-3(4.3)$ & $1.28 \mathrm{E}-3(2.3)$ \\
\hline & Test Eff. (\% 2 s.d.) & $1.66 \mathrm{E}-3(9.0)$ & $1.97 \mathrm{E}-3(9.1)$ & 2.02E-3 (2.5) & $1.55 \mathrm{E}-3(5.0)$ & 1.47E-3 (4.6) & $1.47 \mathrm{E}-3(2.1)$ \\
\hline & Ratio +/- \% 2 s.d. & $1.14+/-12 \%$ & $1.17+/-13 \%$ & $1.20+/-3.8 \%$ & $1.19+/-6.9 \%$ & $1.15+/-6.3 \%$ & $1.15+/-3.1 \%$ \\
\hline \multirow[t]{3}{*}{ Lung } & Ref. Eff. (\% 2 s.d.) & $2.57 \mathrm{E}-4(2.8)$ & $2.33 \mathrm{E}-4(6.5)$ & $1.94 \mathrm{E}-4(3.1)$ & $1.24 \mathrm{E}-4(5.1)$ & $1.11 \mathrm{E}-4(4.9)$ & $9.66 \mathrm{E}-5(4.4)$ \\
\hline & Test Eff. (\% 2 s.d.) & $2.15 \mathrm{E}-4(6.6)$ & 2.09E-4 (14.2) & $1.83 \mathrm{E}-4(5.7)$ & 1.03E-4 (11.5) & $1.01 \mathrm{E}-4(10.6)$ & $8.81 \mathrm{E}-5(8.6)$ \\
\hline & Ratio +/- \% 2 s.d. & $0.84+/-7.2 \%$ & $0.90+/-16 \%$ & $0.94+/-6.5$ & $0.83+/-13 \%$ & $0.91+/-12 \%$ & $0.91+/-9.7 \%$ \\
\hline \multirow[t]{3}{*}{ Total Body } & Ref. Eff. (\% 2 s.d.) & $2.52 \mathrm{E}-4(4.3)$ & 2.03E-4 (8.5) & $1.80 \mathrm{E}-4(3.7)$ & $1.21 \mathrm{E}-4(6.6)$ & $9.52 \mathrm{E}-5(8.2)$ & $8.09 \mathrm{E}-5(6.0)$ \\
\hline & Test Eff. (\% 2 s.d.) & $1.98 \mathrm{E}-4(4.0)$ & $1.94 \mathrm{E}-4(8.5)$ & $1.52 \mathrm{E}-4(4.1)$ & $1.07 \mathrm{E}-4(7.4)$ & $9.41 \mathrm{E}-5(7.4)$ & $7.75 \mathrm{E}-5(6.3)$ \\
\hline & Ratio +/- \% 2 s.d. & $0.79+/-5.9 \%$ & $0.96+/-12 \%$ & $0.84+/-5.5 \%$ & $0.88+/-9.9 \%$ & $0.99+/-11 \%$ & $0.96+/-8.7 \%$ \\
\hline \multirow[t]{3}{*}{ GI } & Ref. Eff. (\% 2 s.d.) & $2.59 \mathrm{E}-4(3.3)$ & $2.21 \mathrm{E}-3(7.4)$ & $1.84 \mathrm{E}-3(3.4)$ & $1.21 \mathrm{E}-3(6.2)$ & $1.05 \mathrm{E}-4(7.2)$ & $8.93 \mathrm{E}-5(5.4)$ \\
\hline & Test Eff. (\% 2 s.d.) & $2.59 \mathrm{E}-4(3.8)$ & $2.32 \mathrm{E}-3(7.8)$ & $2.07 \mathrm{E}-3(3.5)$ & $1.24 \mathrm{E}-3(6.6)$ & $1.22 \mathrm{E}-4(6.1)$ & $1.06 \mathrm{E}-4(5.2)$ \\
\hline & Ratio +/- \% 2 s.d. & $1.00+/-5.0 \%$ & $1.05+/-11 \%$ & $1.12+/-4.9 \%$ & $1.02+/-9.1 \%$ & $1.16+/-9.4 \%$ & $1.19+/-7.5 \%$ \\
\hline
\end{tabular}


Table J-2. Comparison of RMC-II and ANSI N13.30 reference phantoms efficiency ratio values and corresponding relative bias values.

\begin{tabular}{|c|c|c|c|}
\hline Counter Configuration & Source Type & $\begin{array}{l}\text { Range of Efficiency } \\
\text { Ratio Value } 00 \mathrm{~s}^{\mathrm{a}}\end{array}$ & $\begin{array}{c}\text { Range of Measured } \\
\text { Activity } \\
\text { Relative Bias Values }^{\mathrm{b}}\end{array}$ \\
\hline \multirow[t]{3}{*}{ Fastscan } & Lung & 0.92 to 1.04 & -0.04 to 0.09 \\
\hline & Total Body & 1.03 to 1.15 & -0.13 to -0.03 \\
\hline & G.I. Region & 0.99 to 1.16 & -0.14 to 0.01 \\
\hline \multirow{3}{*}{$\begin{array}{l}\text { Accuscan Bed } \\
\text { (Full Bed Scan Mode) }\end{array}$} & Lung & 0.82 to 0.98 & 0.02 to 0.22 \\
\hline & Total Body & 0.97 to 1.17 & -0.15 to 0.03 \\
\hline & G.I. Region & 1.14 to 1.20 & -0.17 to -0.12 \\
\hline \multirow{3}{*}{$\begin{array}{l}\text { Accuscan-II } \\
\text { (Full Shield Scan Mode) }\end{array}$} & Lung & 0.83 to 0.94 & 0.06 to 0.20 \\
\hline & Total Body & 0.79 to 0.99 & 0.01 to 0.27 \\
\hline & G.I. Region & 1.00 to 1.19 & -0.16 to 0.00 \\
\hline \multicolumn{4}{|c|}{$\begin{array}{l}\text { a. Efficiency Ration }=(\text { RMC-II phantom Effic. }) /(\text { Reference phantom Effic.) } \\
\text { (Range shows lowest and highest values listed in Table J-1 for photon energies between } 122 \text { and } \\
1408 \text { keV.) }\end{array}$} \\
\hline \multicolumn{4}{|c|}{ b. $\quad$ Relative Bias $=(\mathbf{A} 2-\mathbf{A} 1) /(\mathbf{A 1})$} \\
\hline \multicolumn{4}{|c|}{$\begin{array}{l}\mathrm{A} 1=\text { measured activity value if reference phantom used for efficiency calibration } \\
\mathrm{A} 2=\text { measured activity value if RMC-II phantom used for efficiency calibration }\end{array}$} \\
\hline
\end{tabular}




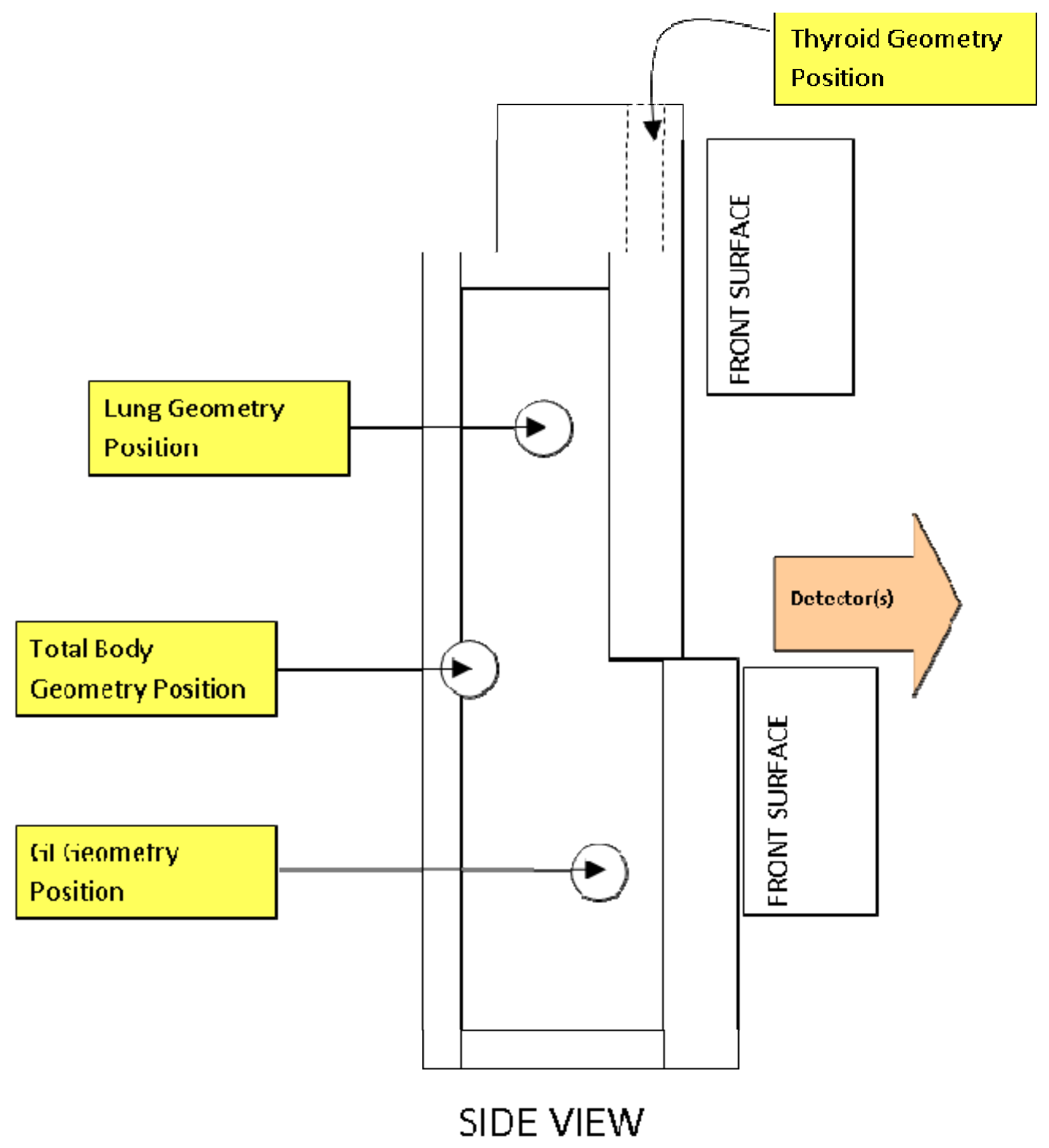

Figure J-1. Canberra RMS-II (Model 2257) WBC calibration phantom. 